\title{
High-resolution peripheral quantitative computed tomography in patients with rheumatoid arthritis
}

Citation for published version (APA):

Scharmga, A. M. J. (2017). High-resolution peripheral quantitative computed tomography in patients with rheumatoid arthritis: truth, discrimination and feasibility. [Doctoral Thesis, Maastricht University]. Maastricht University. https://doi.org/10.26481/dis.20171221as

Document status and date:

Published: 01/01/2017

DOI:

10.26481/dis.20171221as

Document Version:

Publisher's PDF, also known as Version of record

\section{Please check the document version of this publication:}

- A submitted manuscript is the version of the article upon submission and before peer-review. There can be important differences between the submitted version and the official published version of record.

People interested in the research are advised to contact the author for the final version of the publication, or visit the DOI to the publisher's website.

- The final author version and the galley proof are versions of the publication after peer review.

- The final published version features the final layout of the paper including the volume, issue and page numbers.

Link to publication

\footnotetext{
General rights rights.

- You may freely distribute the URL identifying the publication in the public portal. please follow below link for the End User Agreement:

www.umlib.nl/taverne-license

Take down policy

If you believe that this document breaches copyright please contact us at:

repository@maastrichtuniversity.nl

providing details and we will investigate your claim.
}

Copyright and moral rights for the publications made accessible in the public portal are retained by the authors and/or other copyright owners and it is a condition of accessing publications that users recognise and abide by the legal requirements associated with these

- Users may download and print one copy of any publication from the public portal for the purpose of private study or research.

- You may not further distribute the material or use it for any profit-making activity or commercial gain

If the publication is distributed under the terms of Article $25 \mathrm{fa}$ of the Dutch Copyright Act, indicated by the "Taverne" license above, 
High-resolution peripheral quantitative computed tomography in patients with rheumatoid arthritis:

truth, discrimination and feasibility 
C) Andrea Scharmga, Maastricht 2017

All rights reserved. No part of this book may be reproduced or transmitted in any form or by any means, without prior permission in writing by the author, or when appropriate, by the publishers of the publications.

Design: Loch Ard Gorge, Great Ocean Road, Victoria, Australia, with professional execution by Remko Rinia (www.linecreation.nl)

Production: Gildeprint - www.gildeprint.nl

ISBN/ EAN: 9789462337510

Printing was financially supported by Maastricht University, Pfizer, Scanco Medical and the Dutch Arthritis Foundation 


\section{High-resolution peripheral quantitative computed tomography in patients with rheumatoid arthritis: truth, discrimination and feasibility}

\section{PROEFSCHRIFT}

ter verkrijging van de graad van doctor aan de Universiteit Maastricht, op gezag van de Rector Magnificus, Prof. dr. Rianne M. Letschert, volgens het besluit van het College van Decanen, in het openbaar te verdedigen op donderdag 21 december 2017 om 12.00 uur

door

Andrea Maatje Jacomina Scharmga 
Promotores

Prof. dr. P.P.M.M. Geusens

Prof. dr. J.P. van den Bergh

Copromotores

Dr. A. van Tubergen

Dr. ir. B. van Rietbergen, TU Eindhoven

Leden van de beoordelingscommissie

Prof. dr. L. van Rhijn (voorzitter)

Prof. dr. A. Boonen

Prof. dr. R. van der Hulst

Prof. dr. ir. K. Ito, TU Eindhoven

Prof. dr. G. Kloppenburg, LUMC Leiden 


\section{Content}

Chapter 1 General introduction 7

Chapter 2 Heterogeneity of cortical breaks in hand joints of patients with rheumatoid arthritis and healthy controls imaged by HighResolution peripheral Quantitative Computed Tomography

Chapter 3 Visual Detection of Cortical Breaks in Hand Joints: Reliability 31 and Validity of High-Resolution peripheral Quantitative CT compared to MicroCT

Chapter 4 Vascular channels in metacarpophalangeal joints: a 45 comparative histologic and high-resolution imaging study

Chapter 5 Development of a scoring method to visually score cortical interruptions on High-Resolution peripheral Quantitative Computed Tomography in rheumatoid arthritis and healthy controls

Chapter 6 Structural damage and inflammation on radiographs or MRI are associated with cortical interruptions on high-resolution peripheral quantitative CT: a study in finger joints of patients with rheumatoid arthritis and healthy subjects

Chapter 7 An automated algorithm for the detection of cortical interruptions on High Resolution peripheral Quantitative Computed Tomography images of finger joints

Chapter 8 Discussion and summary

Chapter 9 Addendum

Valorisation

Nederlandse samenvatting

Dankwoord

Curriculum Vitae 

General introduction 


\section{Scope}

About one percent of the population, mostly females, is affected by the chronic potentially disabling inflammatory disease rheumatoid arthritis (RA) ${ }^{1}$. Symptoms such as pain and stiffness of the joints start over weeks to months, and radiological evidence of structural damage to joints of hands and feet can be seen early in the disease course, and even before the occurrence of clinical symptoms ${ }^{1-3}$. Structural damage contributes to loss of function at a later stage ${ }^{4,5}$. Hence imaging plays an essential role in the evaluation of RA. Currently, conventional radiography (CR) of the hands and feet are the gold standard in the assessment of joint damage in clinical practice and research-9. Although inexpensive and fast to perform, the use of CR has disadvantages. Only a minority of patients with RA has lesions in small joints on CR at diagnosis ${ }^{10-12}$. Furthermore, the detection of progression of structural damage over time is slow, projectional superimposition may impede scoring of lesions, and scoring can be time consuming 9 . In the case of some clinical trials using CR, larger sample sizes are needed in order to observe a radiographic effect ${ }^{13}$. High-Resolution peripheral Quantitative Computed Tomography (HR-pQCT) is a novel imaging technique that allows detailed evaluation of cortical and trabecular structures. HR-pQCT has originally been developed to study bone architecture at the distal radius and tibia in more detail in the context of metabolic bone diseases, including osteoporosis, and can be combined with structural mechanics analyses to calculate bone strength ${ }^{14-16}$. HR-pQCT also has the potential to identify and quantify early bone changes in metacarpophalangeal (MCP) joints before permanent bone damage occurs ${ }^{17}$. Due to the high spatial resolution with an 82 microns isotropic voxel size, it may contribute to the understanding of bone and joint diseases, such as RA, at the micro-architectural level ${ }^{18}$. In this thesis, we studied the validity, reliability, and feasibility of HR-pQCT in the detection of cortical bone morphologic characteristics of MCP and proximal interphalangeal (PIP) joints, in terms of the detection and significance of cortical interruptions, in healthy subjects and in patients with RA.

\section{Rheumatoid arthritis and imaging}

The presence of peri-articular interruptions in the cortex is a central feature of bone involvement in RA. Due to inflammation, the formation of osteoclasts and suppression of osteoblasts in the bone microenvironment of finger joints cause a net loss of bone and bone destruction, which rarely repairs ${ }^{4}$. Second and third MCP joints are among the first finger joints to be affected, with - as demonstrated on CR - the ulnar and radial side of these joints most commonly involved ${ }^{6}$. The rate of progression of interruptions is highest early in the disease course of $\mathrm{RA}^{2}$. Therefore, the presence, number and size of large interruptions have diagnostic and prognostic implications ${ }^{6}$. Moreover, in the evaluation of therapeutic responses to drugs, progression of radiographic damage in the joints has served as a valuable outcome measure ${ }^{19}$. In the management of patients with RA, sensitive imaging techniques are suggested for diagnosis, monitoring of disease activity and damage, and prognostication ${ }^{20}$. The European League Against 
Rheumatism recommendations for the use of imaging of joints in the clinical management of RA propose that CR of the hands and feet should be used as initial imaging technique to detect damage ${ }^{21}$. In absence of abnormalities on $C R$, ultrasound and/or MRI could be considered ${ }^{21}$.

Peri-articular bone lesions, defined as erosions on $\mathrm{CR}$, reflect a history of inflammation that resulted in bone destruction. CT, MRI and ultrasound (US) are more sensitive than $\mathrm{CR}$ in detecting early structural changes in the joints ${ }^{20,22-24}$. Also, three-dimensional visualization is possible with multiplanar reconstruction, compared to the twodimensional visualization of CR. Clinical CT appears to be more sensitive in detecting structural damage compared to MRI because it is better able to delineate cortical bone margins ${ }^{23-26}$. MRI and US have, in contrast to $C T$ and $C R$, also the ability to visualize soft tissue, enabling early detection of inflammatory changes ${ }^{23,27-29}$. With MRI bone marrow edema (BME) can be visualized, which cannot be detected with other imaging modalities. BME is the reflection of inflammation in the bone and is considered to be one of the links between inflammation and bone destruction $27,30,31$.

There are also some disadvantages to the (sole) use of US and MRI. With US, it is not possible to penetrate the bone, high-frequency probes are required for optimal examination of the small joints of the finger, the use is operator dependent, and standardization and validation are still incomplete ${ }^{20}$. The use of MRI is expensive, is time consuming and its availability may be limited. Furthermore, interruptions can be mimicked by lesions, such as focal bone edema or bone sclerosis, that do not involve bone loss, potentially leading to overestimation of the interruption size on MRI compared to conventional $\mathrm{CT}^{24,25}$.

\section{Imaging of finger joints by HR-pQCT}

The use of HR-pQCT imaging of peri-articular regions in finger joints has been investigated in several domains such as the quantification of bone changes, measurements of size of cortical interruptions, and assessment of the anatomical sites prone to cortical interruptions ${ }^{17,32-35}$. With HR-pQCT it is feasible to detect very small cortical interruptions $(<0.5 \mathrm{~mm}$ in diameter). Cortical interruptions can be found in healthy individuals, however the size and number of these interruptions is greater in $\mathrm{RA}^{17,33}$. Cortical interruptions sized $\geq 1.9 \mathrm{~mm}$ are considered specific for RA ${ }^{17,33}$. An image acquisition protocol has been proposed by the international research collaboration Study grouP for xtrEme Computed Tomography in Rheumatoid Arthritis (SPECTRA), in order to analyze the HR-pQCT images in MCP joints in inflammatory $\operatorname{arthritis}^{34}$.

\section{Validity, reliability and feasibility}

Outcome Measures in Rheumatology (OMERACT), an independent initiative of health professionals interested in outcome measures in rheumatology, strives to improve endpoint outcome measurement through a data driven, iterative consensus process. 
According to the OMERACT filter, an instrument needs to meet the requirements defined by truth, discrimination and feasibility ${ }^{36,37}$. Hence, the application of HR-pQCT for imaging of finger joints should also be reflected by its validity, reliability and feasibility. This raises the question whether HR-pQCT measures what is intended to measure in a reliable and feasible manner.

\section{'Truth' of HR-pQCT}

With 'truth', questions such as "Is the measure truthful, does it measure what is intended?" and "Is the result unbiased and relevant?" are answered"36. On HR-pQCT, different aspects of bone in finger joints can be detected, such as physiologic vascular channels but also pathologic abnormalities such as erosions, specific for RA, and osteophytes, as seen in osteoarthritis ${ }^{38}$. However, visually identifying and interpreting abnormalities in the cortex at high resolution is a complex task ${ }^{39}$.

Several definitions for these various types of cortical interruptions have been proposed in the literature and by SPECTRA $32,38,40$. According to SPECTRA, a cortical interruption is considered an erosion when the cortical bone surface is accompanied by loss of adjacent trabecular bone ${ }^{40}$ or as a sharply marginated bone lesion with juxta-articular localization with a cortical break seen in at least two adjacent slices ${ }^{32}$. A cortical interruption is considered a vascular channel when there is a parallel structure and presence of normal trabecular structure or when there is a small cortical tunnel, which is typically located at the palmar side of the metacarpal head and phalangeal base ${ }^{38}$.

In a barium perfusion study in two vascular channels in one hand, Boutroy et al. showed that the SPECTRA definition for vascular channel on HR-pQCT could be supported ${ }^{41}$. However, no evidence for the correct interpretation of HR-pQCT imaging of finger joints is available from gold standards, such as microCT $(\mu C T)$, which can obtain much higher resolutions, or histology.

\section{'Discrimination' of HR-pQCT}

With 'discrimination', a question such as "Does the measure discriminate between situations of interest?" is answered ${ }^{36}$. Measures of reliability, sensitivity and specificity show the ability of a measurement instrument to provide similar results upon replication, and the effectiveness of a test to correctly identify the measurement of interest. First steps in evaluating the ability of HR-pQCT to detect cortical interruptions have already been taken for discrimination between RA and healthy controls, healing of interruptions, methods for quantification of large interruptions, and for automated analysis 17,32,33,35,42. Scoring methods used in HR-pQCT image analysis showed that semi-quantitative grading of large interruptions had good intra- and inter-reader reliability (kappa (K) 0.82 and 0.75 respectively) ${ }^{17}$. Also, almost perfect intra- and inter-reader reliability results were observed on maximal interruption dimension measurements (ICC 0.89 and 0.99 respectively) ${ }^{33}$. Furthermore, HR-pQCT is able to show evidence of repair of interruptions in patients with RA treated with tumor necrosis factor inhibitors (TNFi) compared to patients with RA treated with methotrexate ${ }^{42}$. After one year, the mean 
depth of the interruptions significantly decreased in the TNFi treated group $(-0.10 \pm$ $0.04 \mathrm{~mm}$; median $-0.03 \mathrm{~mm}<<0.016)$ compared to an increase of mean depth of the interruptions in the methotrexate group $1+2.16 \pm 0.24 \mathrm{~mm}$; median $+1.95 \mathrm{~mm}$, $p<0.001)^{42}$. Also, an automated method to measure interruption volume, surface area and shape, but in which the operator still needs to define the interruption, has been tested ${ }^{35}$. It was compared to manual size measurements and a correlation of $r=0.61$ was found ${ }^{35}$. Inter-operator precision errors for calculated volumes were $15.39 \%$ (coefficient of variation) and $0.36 \mathrm{~mm}^{3}$ (root mean square of the standard deviation) ${ }^{35}$.

These few studies focused on large interruptions. Small cortical interruptions have not been studied so far and their role in RA is unknown; they might be physiological, representing vascular channels or pathological, representing the early stage of an erosion. The ability of HR-pQCT to distinguish between physiological or pathological cortical interruptions therefore needs to be studied by comparison of HR-pQCT with $\mu \mathrm{CT}$ and histology. Subsequently, based on these findings, a visual or automated scoring algorithm could be developed, which is able to distinguish between patients with RA and healthy subjects.

\section{'Feasibility' of HR-pQCT}

With 'feasibility', the question "Can the measure be applied easily, given constraints of time, money, and interpretability?" is answered ${ }^{36}$. Scan duration of second and third MCP and PIP joints of one hand is approximately 15 minutes to cover an area of 27.06 $\mathrm{mm}$ (MCP joints) and $18.04 \mathrm{~mm}$ (PIP joints). During scanning with HR-pQCT, the patient sits upright in a chair, with the arm being scanned, immobilized but resting, in a carbon fiber cast. Compared to MRI where scanning of both hands with contrast agents takes up to one hour, HR-pQCT scanning takes less time and administration of a contrast agent is not necessary. Compared to US, HR-pQCT imaging is not operator dependent. Another important aspect is the time to score the images. Only one study has reported data on the feasibility of HR-pQCT image analysis using a quantitative method for measurement of interruptions in RA. The median time to evaluate images was 2 minutes (range $1.20-5.30$ minutes) for a single MCP joint ${ }^{33}$. It is unknown what time it takes to analyze the HR-pQCT images in detail, including all types of (small) cortical interruptions.

\section{Aims of this thesis}

This thesis tries to answer several open questions regarding 'truth', 'discrimination' and 'feasibility' of HR-pQCT in the detection of cortical interruptions in peri-articular regions of finger joints of patients with RA and healthy subjects. In particular, we first aimed to describe the heterogeneous spectrum of visually detected cortical interruptions in finger joints of both healthy subjects and patients with RA, subsequently to study the truth and reliability by comparing visually detected cortical interruptions on HR-pQCT with $\mu C T$ as gold standard, and by matching cortical interruptions on HR-pQCT images with corresponding histologic sections. As a next step, we aimed to develop a visual scoring 
method for detection of cortical interruptions and to test its truth and feasibility. Then, we applied this visual scoring method on HR-pQCT images of fingers joints of patients with RA and healthy subjects, to study the relationship between structural bone and/or inflammatory features on either MRI or radiography and other risk factors (anticitrullinated protein antibody (ACPA) and/or rheumatoid factor (RF) seropositivity, hand dominance, disease duration) with the presence or number of cortical interruptions in finger joints on HR-pQCT. Finally, an automated algorithm to detect cortical interruptions was developed and its truth was tested.

\section{Outline of this thesis}

At the Maastricht University Medical Center, the Netherlands, 38 female healthy subjects and 41 female patients diagnosed with RA were recruited to study the spectrum of bone abnormalities, and to develop and validate a visual and automated HR-pQCT scoring algorithm. For the studies using $\mu C T$ and histology, ten female index fingers from anatomic specimens from the Department of Anatomy and Embryology of the University of Amsterdam, the Netherlands, were used. Using patients, healthy subjects and anatomic specimens gave us the possibility to study cortical interruptions among a wide range of imaging techniques (CR, MRI, HR-pQCT and $\mu C T)$ and histology. In chapter 2 of this thesis, the heterogeneous spectrum of cortical interruptions seen in MCP joints of patients with RA and healthy subjects imaged with HR-pQCT is presented. In chapter 3, the truth and reliability of HR-pQCT in the visual detection of cortical interruptions compared with $\mu C T$ as a gold standard is described. In chapter 4, we evaluated whether cortical interruptions classified as vascular channels on HR-pQCT could be confirmed by histology. We subsequently evaluated the image characteristics of histologically identified vascular channels on matched single and multiplane HR-pQCT images. Chapters 5 and 7 focus on the development, truth and feasibility of respectively a visual and an automated scoring algorithm for HRpQCT images. In chapter 6 associations of structural damage on MRI and radiography, markers of inflammation on MRI, and other risk factors are studied in relation to the presence and number of cortical interruptions on HR-pQCT in our cohort of healthy subjects and patients with RA. In chapter 8 , the overall findings from this thesis are summarized and discussed. 


\section{References}

1 Paleolog, E. M. The vasculature in rheumatoid arthritis: cause or consequence? Int J Exp Pathol 90, 249261 (2009).

2

3

4

5

6

8 van der Heijde, D. M. Joint erosions and patients with early rheumatoid arthritis. Br J Rheumato/ 34 Suppl 2, 74-78 (1995).

Kleyer, A. et al. Bone loss before the clinical onset of rheumatoid arthritis in subjects with anticitrullinated protein antibodies. Ann Rheum Dis (2013).

Gravallese, E. M. \& Walsh, N. C. Rheumatoid arthritis: Repair of erosion in RA-shifting the balance to formation. Nat Rev Rheumatol 7, 626-628 (2011).

Lillegraven, S., van der Heijde, D., Uhlig, T., Kvien, T. K. \& Haavardsholm, E. A. What is the clinical relevance of erosions and joint space narrowing in RA? Nat Rev Rheumatol 8, 117-120 (2012).

Sommer, O. J. et al. Rheumatoid arthritis: a practical guide to state-of-the-art imaging, image interpretation, and clinical implications. Radiographics 25, 381-398 (2005).

Boini, S. \& Guillemin, F. Radiographic scoring methods as outcome measures in rheumatoid arthritis: properties and advantages. Ann Rheum Dis 60, 817-827 (2001).

van der Heijde, D., Dankert, T., Nieman, F., Rau, R. \& Boers, M. Reliability and sensitivity to change of a simplification of the Sharp/van der Heiide radiological assessment in rheumatoid arthritis. Rheumatology (Oxford) 38, $941-947$ (1999).

Ravindran, V. \& Rachapalli, S. An overview of commonly used radiographic scoring methods in rheumatoid arthritis clinical trials. Clin Rheumato/ 30, 1-6 (2011).

Mottonen, T. T. Prediction of erosiveness and rate of development of new erosions in early rheumatoid arthritis. Ann Rheum Dis 47, 648-653 (1988).

van der Heijde, D. M. et al. Biannual radiographic assessments of hands and feet in a three-year prospective followup of patients with early rheumatoid arthritis. Arthritis Rheum 35, 26-34 (1992).

Nissila, M. et al. Prognosis of inflammatory joint diseases. A three-year follow-up study. Scand J Rheumatol 12, 33-38 (1983).

Sokka, T. Radiographic scoring in rheumatoid arthritis: a short introduction to the methods. Bull NYU Hosp Jt Dis 66, 166-168 (2008).

Boutroy, S., Bouxsein, M. L., Munoz, F. \& Delmas, P. D. In vivo assessment of trabecular bone microarchitecture by high-resolution peripheral quantitative computed tomography. J Clin Endocrinol Metab 90, 6508-6515 (2005).

Laib, A., Hauselmann, H. J. \& Ruegsegger, P. In vivo high resolution 3D-QCT of the human forearm. Technol Health Care 6, 329-337 (1998).

MacNeil, J. A. \& Boyd, S. K. Accuracy of high-resolution peripheral quantitative computed tomography for measurement of bone quality. Med Eng Phys 29, 1096-1 105 (2007).

Stach, C. M. et al. Periarticular bone structure in rheumatoid arthritis patients and healthy individuals assessed by high-resolution computed tomography. Arthritis Rheum 62, 330-339 (2010).

Geusens, P. et al. High-resolution in vivo imaging of bone and joints: a window to microarchitecture. Nat Rev Rheumato/ 10, 304-313 (2014).

Boers, M. et al. World Health Organization and International League of Associations for Rheumatology core endpoints for symptom modifying antirheumatic drugs in rheumatoid arthritis clinical trials. J Rheumatol Supp/ 41, 86-89 (1994).

Ostergaard, M., Pedersen, S. J. \& Dohn, U. M. Imaging in rheumatoid arthritis-status and recent advances for magnetic resonance imaging, ultrasonography, computed tomography and conventional radiography. Best Pract Res Clin Rheumatol 22, 1019-1044 (2008).

1 Colebatch, A. N. et al. EULAR recommendations for the use of imaging of the joints in the clinical management of rheumatoid arthritis. Ann Rheum Dis 72, 804-814 (2013).

Baillet, A. et al. Comparison of the efficacy of sonography, magnetic resonance imaging and conventional radiography for the detection of bone erosions in rheumatoid arthritis patients: a systematic review and meta-analysis. Rheumatology (Oxford) 50, $1137-1147$ (2011).

Dohn, U. M. et al. Are bone erosions detected by magnetic resonance imaging and ultrasonography true erosions? A comparison with computed tomography in rheumatoid arthritis metacarpophalangeal joints. Arthritis Res Ther 8, R1 10 (2006).

Perry, D. et al. Detection of erosions in the rheumatoid hand; a comparative study of multidetector computerized tomography versus magnetic resonance scanning. J Rheumato/ 32, 256-267 (2005). Dohn, U. M. et al. Rheumatoid arthritis bone erosion volumes on CT and MRI: reliability and correlations with erosion scores on CT, MRI and radiography. Ann Rheum Dis 66, 1388-1392 (2007).

Dohn, U. M. et al. Detection of bone erosions in rheumatoid arthritis wrist joints with magnetic resonance imaging, computed tomography and radiography. Arthritis Res Ther 10, R25 (2008). Conaghan, P. G et al. Elucidation of the relationship between synovitis and bone damage: a randomized magnetic resonance imaging study of individual joints in patients with early rheumatoid arthritis. Arthritis Rheum 48, 64-71 (2003).

8 Ostergaard, M. et al. Magnetic resonance imaging-determined synovial membrane and joint effusion volumes in rheumatoid arthritis and osteoarthritis: comparison with the macroscopic and microscopic appearance of the synovium. Arthritis Rheum 40, 1856-1867 (1997). 
sensitive detection of morphologic changes in rheumatoid arthritis. Arthritis Rheum 44, 2492-2502 (2001). McGonagle, D. et al. The relationship between synovitis and bone changes in early untreated rheumatoid arthritis: a controlled magnetic resonance imaging study. Arthritis Rheum 42, 1706-1711 (1999).

31 Krabben, A. et al. MRI-detected subclinical joint inflammation is associated with radiographic progression. Ann Rheum Dis 73, 2034-2037 (2014).

Fouque-Aubert, A. et al. Assessment of hand bone loss in rheumatoid arthritis by high-resolution peripheral quantitative CT. Ann Rheum Dis 69, 1671-1676 (2010).

33 Srikhum, W. et al. Quantitative and semiquantitative bone erosion assessment on high-resolution peripheral quantitative computed tomography in rheumatoid arthritis. J Rheumatol 40, 408-4 16 (2013).

34 Barnabe, C. \& Feehan, L. High-resolution peripheral quantitative computed tomography imaging protocol for metacarpophalangeal joints in inflammatory arthritis: the SPECTRA collaboration. J Rheumatol 39, 1494-1495 (2012).

Topfer, D., Finzel, S., Museyko, O., Schett, G. \& Engelke, K. Segmentation and quantification of bone erosions in high-resolution peripheral quantitative computed tomography datasets of the metacarpophalangeal joints of patients with rheumatoid arthritis. Rheumatology (Oxford) 53, 65-71 (2014). Boers, M., Kirwan, J. R. \& Tugwell, P. The OMERACT Handbook. (2015). Boers, M., Brooks, P., Strand, C. V. \& Tugwell, P. The OMERACT filter for Outcome Measures in Rheumatology. J Rheumatol 25, 198-199 (1998).

38 Finzel, S. et al. A detailed comparative study of high-resolution ultrasound and micro-computed tomography for detection of arthritic bone erosions. Arthritis Rheum 63, 1231-1236 (2011). Zebaze, R. \& Seeman, E. Cortical bone: a challenging geography. J Bone Miner Res 30, 24-29 (2015). Schett, G. \& Gravallese, E. Bone erosion in rheumatoid arthritis: mechanisms, diagnosis and treatment. Nat Rev Rheumatol 8, 656-664 (2012).

41 Boutroy, S. et al. Erosion or vessel channel? Arthritis Rheumatol (2015).

42 Finzel, S. et al. Repair of bone erosions in rheumatoid arthritis treated with tumour necrosis factor inhibitors is based on bone apposition at the base of the erosion. Ann Rheum Dis 70, 1587-1593 (2011). 
Heterogeneity of cortical breaks in hand joints of patients with rheumatoid arthritis and healthy controls imaged by HighResolution peripheral Quantitative Computed Tomography

A. Scharmga, M. Peters, A. van Tubergen,

J. van den Bergh, C. Barnabe, S. Finzel,

B. van Rietbergen, P. Geusens

J Rheumatol 2016;43;1914-1920 


\begin{abstract}
Objective: Conventional radiographs (CR) of the hands are the gold standard for imaging bone erosions. The presence of bone erosions, reflected by the presence of cortical breaks, is a poor prognostic factor in patients with rheumatoid arthritis (RA). The availability of high-resolution peripheral quantitative computed tomography (HRPQCT) enables detailed investigation of cortical breaks in rheumatic diseases. The aim of this image review is to show HR-pQCT images of the spectrum of cortical breaks with and without underlying trabecular bone changes in metacarpophalangeal (MCP) joints of healthy controls $(\mathrm{HC})$ and patients with RA, with corresponding images on $C R$ and magnetic resonance imaging (MRI).

Methods: Second and third MCP joints of 41 patients (of which 10 were early RA with $\leq 2$ years and 24 longstanding RA with $\geq 10$ years of disease duration) and $38 \mathrm{HC}$ were imaged by CR, MRI and HR-pQCT (XtremeCT1, Scanco Medical AG). Representative images of the spectrum of cortical breaks were selected.

Results: Cortical breaks were found in early and longstanding RA, but also in HC. They were heterogeneous in size, location and number per joint, with a variety of surrounding cortical and underlying trabecular bone characteristics.

Conclusion: Using HR-pQCT images of MCP joints, heterogeneous cortical breaks with and without surrounding trabecular bone changes were found, not only in RA but also in $\mathrm{HC}$. The underlying mechanisms and significance of this spectrum of cortical breaks as found with high 3-D resolution needs further investigation.
\end{abstract}




\section{Introduction}

Bone is one of the critical supporting structures of a normally functioning joint. In normal conditions, the only well-documented peri-articular cortical breaks are related to the presence of blood vessels that perforate bone from the periosteum toward the bone marrow at the epiphyses ${ }^{1}$. In rheumatoid arthritis (RA), pathological cortical breaks, i.e. erosions, can occur in the joints. Complex physical, cellular, and molecular interactions between bone and the immune system, termed osteoimmunology, drive the development of bone erosions through activation of osteoclasts ${ }^{2}$. In early RA, osteoclasts are locally activated by inflammation occurring at the synovium. An alternative hypothesis is that the osteoclasts are activated within the bone marrow by anticyclic citrullinated protein antibodies $^{3}$. Osteoclast activation is the final pathway that results in disruption of the bone matrix, mainly at sites not protected by cartilage and at ligament insertions, thus allowing a direct anatomical connection between the joint space and the immune system in the bone marrow, in addition to normal vascular connections at the epiphyses ${ }^{1}$. Second and third metacarpophalangeal (MCP) joints are among the first hand joints affected in the early disease course of RA, with the ulnar and radial side of these joints most commonly involved, as demonstrated on conventional radiographs $(C R)^{4}$. Additional damage can ensue if the inflammatory cascade is not suppressed with therapy. Later in the course of RA, the increasing size of cortical breaks - which rarely heal in RA - contribute to joint damage and loss of function ${ }^{5,6}$.

In RA, the presence, size, and number of cortical breaks, defined as erosions on CR, are poor prognostic factors, and influence the aggressiveness of treatment required ${ }^{4}$. Prevention of these cortical breaks and their progression is one of the major endpoints in clinical trials ${ }^{5}$. Currently, CR of the hands and feet are the gold standard for imaging erosions in both research and clinical practice settings $s^{4,7-9}$. CR is widely available, fast to perform, cheap, and easy to use ${ }^{10}$. Scoring of those bone erosions on radiographs has a long history of interpretation and standardization?. Scoring methods include simple counts of the presence and number of bone erosions, and scoring levels for their $s^{2} e^{9}$. Limitations of radiography include floor effects (i.e. radiographic visualisation of erosions may occur late in the disease) and ceiling effects (i.e. progression can occur even after the highest radiographic damage score has been assigned), slow change over time, and superimposition?. Furthermore, scoring can be time consuming.

High-resolution peripheral quantitative computed tomography (HR-pQCT) is a noninvasive imaging technique. Its spatial resolution allows detailed evaluation of the 3-D cortical and trabecular bone (combined as well as separately). For a standard scan of nine millimetres, the radiation dose $<3 \mu \mathrm{Sv}$. It has been applied for assessment of bone damage in rheumatic diseases affecting the hand joints ${ }^{11}$. In RA, this technology has been shown to be more sensitive than $C R$ for identifying cortical breaks ${ }^{12}$. In addition, HR-pQCT can detect small cortical breaks and exactly localize and measure individual cortical breaks in 3-D, thereby facilitating the monitoring of their development and investigating the effects of antiinflammatory therapies ${ }^{12-14}$. 
The aim of the present HR-pQCT image review is to show the spectrum of cortical breaks on selected images of cortical and subchondral trabecular bone of MCP joints of patients with RA and healthy controls $(\mathrm{HC})$, with corresponding images on $\mathrm{CR}$ and magnetic resonance imaging (MRI). In RA, when scoring peri-articular bone destruction using $C R$ and $M R I$, cortical breaks are considered bone erosions and vice versa. In our presentation, we use the term "cortical break", referring to an image where the cortex is discontinuous, which can be erosions but also vascular channels.

The present review was initiated and supported by SPECTRA (Study grouP for xtrEme Computed Tomography in Rheumatoid Arthritis, an international collaboration of HRpQCT users) in order to expand knowledge and validation in hand joint imaging research in rheumatology ${ }^{15}$.

\section{Materials and methods}

\section{Participants}

Forty-one patients with RA (of which 10 were early RA of $\leq 2 \mathrm{yrs,} \mathrm{and} 24$ longstanding $\mathrm{RA}$ of $\geq 10 \mathrm{yrs}$ of disease duration) and $38 \mathrm{HC}$ were recruited at the Maastricht University Medical Center, the Netherlands. All RA patients fulfilled the 2010 American College of Rheumatology (ACR)/European League Against Rheumatism classification criteria for RA ${ }^{16}$. No HC suffered from hand joint complaints. Baseline and followup (1 yr) measurements of HR-pQCT, MRI and CR were performed. Demographics, disease duration, and presence of rheumatoid factor and anticitrullinated protein antibody were recorded, if applicable. All patients with RA completed the Dutch version of the Health Assessment Questionnaire $(\mathrm{HAQ})^{17}$. All subjects signed informed consent. Ethical approval was obtained from the ethics board of the Academic Hospital Maastricht, the Netherlands.

\section{Conventional radiography}

Posterior-anterior radiographs of both hands of the subjects were taken using highresolution digital radiography (Siemens FD-X $45 \mathrm{kV} / 5 \mathrm{mAs}$ ) according to a standardized protocol in daily practice.

\section{HR-pQCT scanning procedure}

Second and third MCP and proximal interphalangeal (PIP) joints of both hands in RA and only the dominant hand in HC were scanned by HR-pQCT $(82 \mu \mathrm{m}$, XtremeCT1, Scanco Medical AG, Switzerland) according to the standard image acquisition protocol from SPECTRA ${ }^{15}$.

\section{MRI scanning procedure}

The second and third MCP and PIP joints of both hands were examined using a $3.0 \mathrm{~T}$ Achieva Philips MRI device. During the examination, the subject's hand rested in a dedicated wrist coil. The hand was fixed inside the coil and the space around the hand was filled with rubber to reduce motion artefacts. Images were acquired of both hands 
using axial T1-weighted, axial fat-suppressed T2-weighted and sagittal 3D WATSC sequences. Additional images of the hand with most symptoms (in RA) or dominant hand $(\mathrm{HC}$ ) were acquired post-intravenous gadolinium (Gadovist $1.0 \mathrm{mmol} / \mathrm{ml}$ solution for injection) using axial and coronal fat-suppressed T1-weighted images. Total acquisition time was about $30 \mathrm{~min}$ per hand. The MRI images were scored by a radiologist, blinded for clinical data, for the presence of erosions, $B M E$, and synovitis.

\section{Definition of cortical breaks visualized on HR-pQCT}

Regarding the use of $C R$, several definitions for bone erosions are available from the literature, such as cortical break, discontinuous cortex, or defects in contour on an anteroposterior radiograph $12,14,18-20$. Within SPECTRA, a preliminary working definition of cortical breaks on images from HR-pQCT has been developed for application in HRpQCT. In HR-pQCT imaging, once a cortical break is identified on one slice, it is further specified by examining its presence on adjacent slices and specification of subchondral bone characteristics. A cortical break is considered a bone erosion if it is extending over a minimum of 2 consecutive slices in at least 2 perpendicular planes (transverse plane and sagittal or coronal plane); and if it has a nonlinear delineation of the cortical perforation and is accompanied with underlying loss of trabecular structure. A cortical break is considered a vascular channel if it is regularly delineated, without loss of surrounding trabecular structure. A bone cyst is defined as disproportional area of absence of trabecular bone at the site of a small cortical break.

\section{Selection procedure}

One person prepared a selection of typically normal images, variants of normal images, and variants of cortical breaks, according to the proposed SPECTRA definition in the MCP joints. Consensus on the final selection of the images and scoring was reached within a team of experts. The corresponding MRI sequences and radiographs were chosen in collaboration with an MRI technician and a musculoskeletal radiologist. Cases were selected by scrolling through the set of transverse source images from distal to central within the joint (phalangeal base) and proximal to central within the joint (metacarpal head) in a freeware multiplanar reformat DICOM viewer (Osirix v.5.8.5 64-bit). With Osirix, 3 orthogonal planes (transverse, coronal and sagittal) of each joint can simultaneously be viewed. Using a crossed locator, specific locations of interest can be indicated in 1 plane, and this is automatically also indicated on the images in the other 2 planes, as illustrated in Supplementary Figures 2.1 and 2.2, available online at jrheum.org.

The localization of cortical breaks was done as described by Stach, et al. ${ }^{12}$. The localization includes 4 quadrants in transverse view (palmar, ulnar, dorsal and radial), the radial and ulnar side in coronal views, and the palmar and dorsal side in sagittal views, both in the metacarpal head and the phalangeal base (Figure 2.1). In addition, volume measurements can be made in Osirix. Measuring maximal width and depth of a cortical break is done in transverse and coronal or sagittal plane with lines 
perpendicular to each other (Supplementary Figure 2.3, available online at jrheum.org). With HR-pQCT, a 3-D animation of the joint can also be constructed. Two examples of such a 3-D animation of an MCP joint are shown in Supplementary Figure 2.4 and 2.5 (available online at irheum.org). Supplementary Figure 2.4 shows a normal joint, and Supplementary Figure 2.5 shows a joint of an RA patient with cortical breaks compatible with bone erosions.
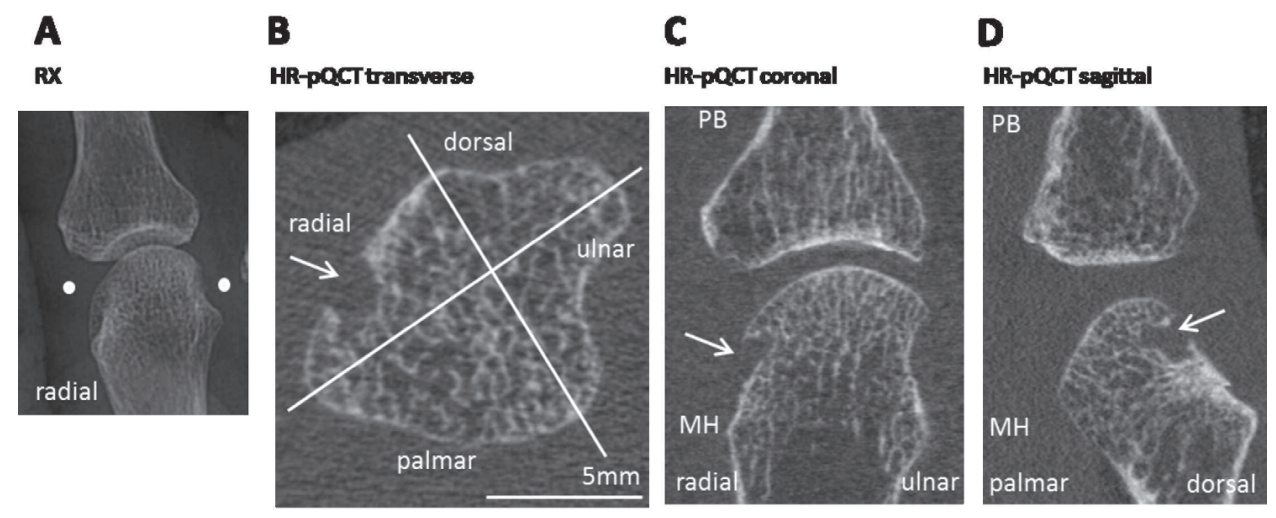

Figure 2.1. MCP joint on $C R(A)$ and HR-pQCT imaging ( $B, C, D)$ of a 62-year-old patient with longstanding RA. The MCP joint is evaluated in transverse view in 4 quadrants (B) and simultaneously viewed in coronal (C) and sagittal (D) planes. A cortical break is seen in the radial quadrant, and in corresponding coronal and sagittal planes. White dots in A correspond to the location of the transverse slice in B. MCP: metacarpophalangeal; CR: conventional radiography; HR-pQCT: high-resolution peripheral quantitative computed tomography; $\mathrm{PB}$ : phalangeal base; $\mathrm{MH}$ : metacarpal head.

\section{Results}

Of the selected subjects, the mean age (SD) of the patients with early RA was 58.5 (0.7) years; patients with longstanding RA: 60.0 (7.0) years; and HC: 53 (13.8) years. The mean (SD) disease duration was 11.5 (10.6) and 226 (84) months for patients with early and longstanding RA, respectively. The mean (SD) Health Assessment Questionnaire scores were $0.31(0.08)$ and $1.93(0.79)$ for early and longstanding RA, respectively.

In HC, we selected HR-pQCT images of normal bone (Figure 2.2) and with cortical breaks (Figure 2.3). In patients with early and longstanding RA we selected images of joints with clear cortical breaks (Figure 2.4 and 2.5). All images are shown with corresponding radiographs and MRI.

\section{Healthy controls}

Characteristic for normal cortical bone is its smooth outer contour without cortical breaks, accompanied by normal subchondral trabecular bone with an evenly distributed branching structure that merges with the cortical bone. Figure 2.2 presents an example of normal cortical bone in an MCP joint of an HC. On CR, no cortical break is visible and on MRI no signs of BME or synovitis. 


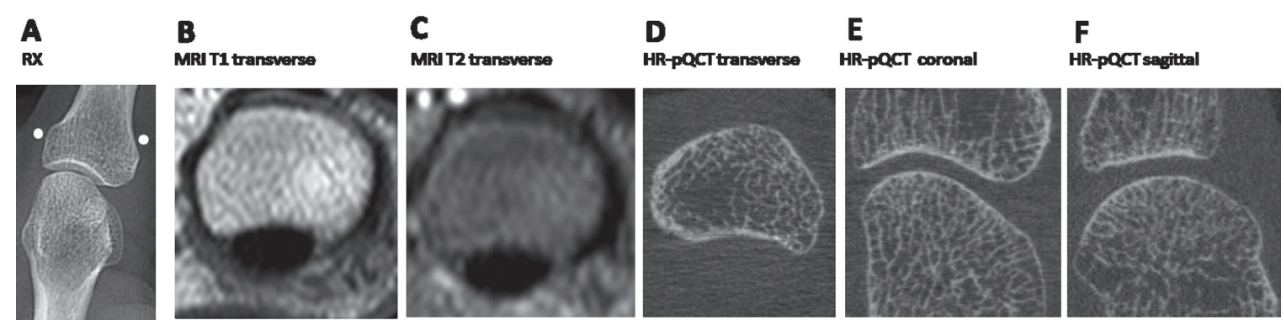

Figure 2.2. Normal MCP joint on CR (A) and HR-pQCT (D, E, F) of a 61 -year-old healthy control. The cortical bone has a smooth outer contour without discontinuities. The normal subcortical trabecular bone shows an evenly distributed branching structure that merges with the cortical bone. The corresponding $M R I$ images $(B, C)$ also show a normal bone without $B M E$ and no signs of synovitis. MCP: metacarpophalangeal; $C R$ : conventional radiography; HR-pQCT: high-resolution peripheral quantitative computed tomography; BME: bone marrow edema.

Nevertheless, cortical breaks were also seen in HC. Figure 2.3 panel 1 shows an example of a cortical break suggestive for a vascular channel, based on the parallel lining of the break and presence of normal trabecular structure (panel 1). This cortical break is not visible on CR and MRI, and there were no signs of BME or synovitis on MRI. Figure 2.3 panel 2 shows an example of a small cortical break with a large area of trabecular bone loss suggesting a subchondral bone cyst which is barely visible on CR but clearly found on MRI without presence of BME and synovitis.

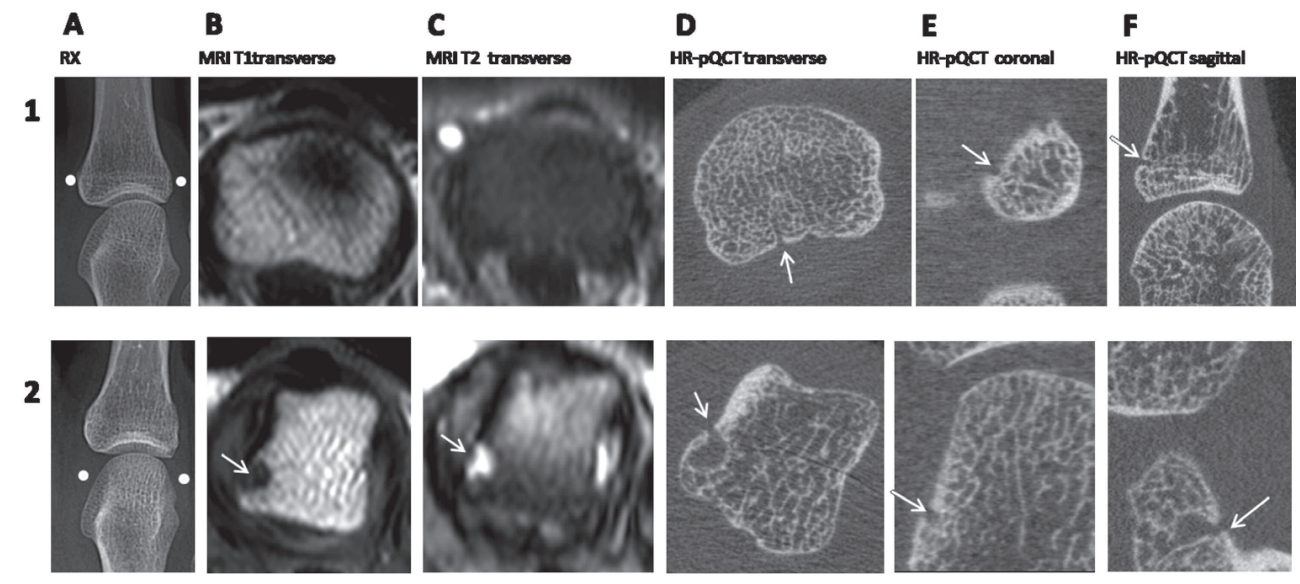

Figure 2.3. Two variants of cortical bone found in MCP joints on $C R(A)$, and HR-pQCT imaging $(D, E$, $F)$, in transverse, coronal, and sagittal views (D, E, F) in healthy controls (panel 1: age 37 years; panel 2: age 61 years). On panel 1, a cortical break is seen on the palmar side, which is suggestive of a vascular channel with no presence of $B M E$ and synovitis on MRI (B, C). Panel 2 shows a subcortical cyst on the radial side without presence of $B M E$ and no synovitis on MRI $(B, C)$. White dots on the $C R$ correspond to the location of the cortical break on HR-pQCT imaging. No abnormalities were found on CR. MCP: metacarpophalangeal; CR: conventional radiography; HR-pQCT: high-resolution peripheral quantitative computed tomography; BME: bone marrow edema; MRI: magnetic resonance imaging. 


\section{Rheumatoid arthritis}

Figure 2.4 shows examples of cortical breaks, considered characteristic for bone erosions, in patients with early RA, at the unar (panel 1), dorsal (panel 2), and radial (panel 3) sides visualised on images from HR-pQCT. Erosions are not visible on CR, but can be seen on MRI, with presence of BME in panel 1 and 2 but without synovitis (all panels).

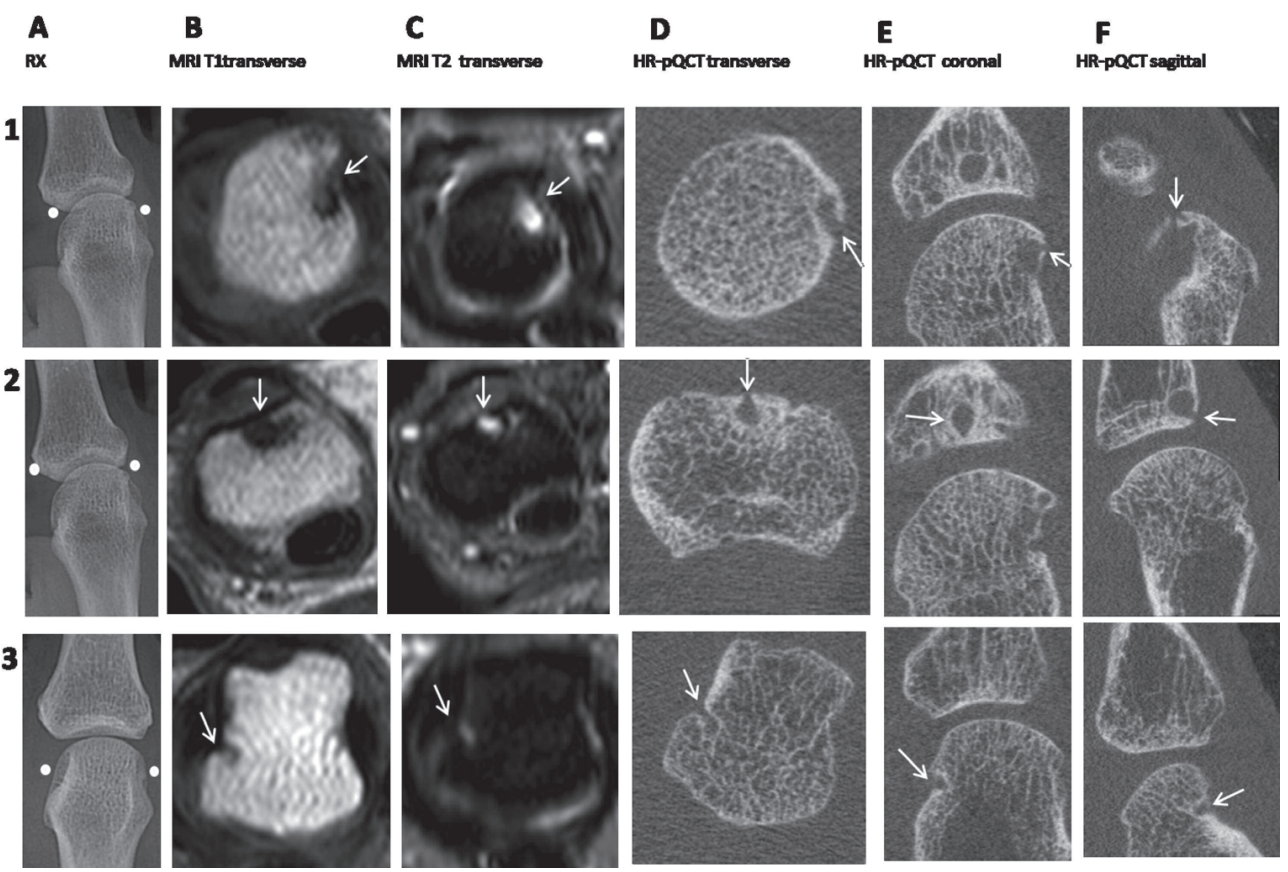

Figure 2.4. No cortical breaks in MCP joints on $C R(A)$ but cortical breaks in MCP joints on MRI $(B, C)$ and HR-pQCT (D, E, F) in patients with early RA (panels 1 and 2: age $59 \mathrm{yrs,} \mathrm{panel} \mathrm{3:} \mathrm{age} 58 \mathrm{yrs}$ ). Disease duration of patient in panels 1 and 2: 19 months (RF-/ACPA-); panel 3: 4 months (RF-/ACPA). BME is seen in panels 1 and 2, but no synovitis on MRI. MCP: metacarpophalangeal; $C R$ : conventional radiography; HR-pQCT: high-resolution peripheral quantitative computed tomography; RF: rheumatoid factor; ACPA: anticitrullinated protein antibodies; BME: bone marrow edema; $M R I$ : magnetic resonance imaging; RA: rheumatoid arthritis.

Figure 2.5 shows 2 examples of patients with longstanding RA with large cortical breaks with irregular surrounding cortical lining and extensive surrounding trabecular bone loss, suggestive for bone erosions. These are also visible on CR and MRI with presence of BME and synovitis on MRI in panel 1 and without presence of BME but presence of synovitis in panel 2 (Figure 2.5). 


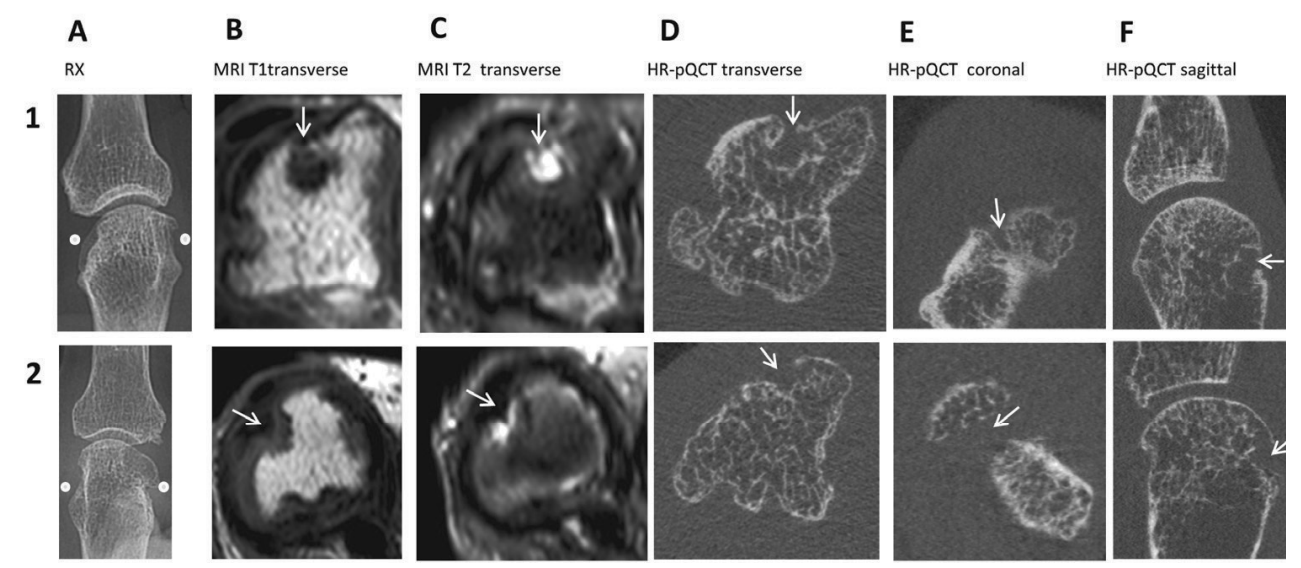

Figure 2.5. Cortical breaks in MCP joints on 3 imaging modalities: $C R(A), M R I(B, C)$, and HR-pQCT $(D, E, F)$ in patients with longstanding RA (panel 1: age $55 \mathrm{yrs}$; panel 2: age $65 \mathrm{yrs}$ ). Disease duration of patient in panel 1: 286 months (RF+/ACPA unknown) and panel 2: 166 months (RF-/ACPA-). Panel 1 shows presence of BME and synovitis on MRI. Panel 2 shows no presence of BME but presence of synovitis on MRI. MCP: metacarpophalangeal; CR: conventional radiography; HR-pQCT: high-resolution peipheral quantitative computed tomography; RA: rheumatoid arthritis; RF: rheumatoid factor; ACPA: anticitrullinated protein antibodies; BME: bone marrow edema; MRI: magnetic resonance imaging.

\section{Discussion}

The HR-pQCT images of MCP joints shown in this image review serve as an illustration of intact cortical bone and heterogeneous cortical breaks found not only in patients with early and longstanding RA, but also in $\mathrm{HC}$.

On HR-pQCT, cortical breaks are found at all sides, i.e., radial, ulnar, palmar and dorsal; and both in the metacarpal head and in the phalangeal base. Breaks vary from very small to very large, and are located close to and/or remote from the joint space. Also, a highly variable damage to the trabecular structure in terms of size and delineation (with or without bone sclerosis) is seen. As expected, the presence and size of bone damage is more pronounced and complex on HR-pQCT than can be observed

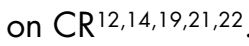

HR-pQCT is a clinically feasible modality, with radiation exposure levels of $<3 \mu \mathrm{Sv}$ and scan time of $9 \mathrm{~min}$ for standard scans, a duration most patients can tolerate. The major advantage over CR is its 3-D resolution. CR provides a projection of spatial structures, which may lead to errors in interpretation due to positioning and superimposition. HRpQCT overcomes this problem, i.e., the examination can be viewed simultaneously in transverse, coronal and sagittal plane, creating a 3-D impression of the joint. Also, it provides opportunities for measuring new, potentially very relevant characteristcs, such as the 3-D joint space volume and cortical break and erosion volume by manual or semiautomatic assessment ${ }^{18,19}$. However, the HR-pQCT also has its limitations, such as its limited availability of the system, the time that is presently involved in scoring and interpreting the images, operator-dependent variation when a cortical break is considered an erosion, and movement artefacts, which can reduce the accuracy ${ }^{23}$. 
Further, although scan time of the joints is relatively short, only a few joints are imaged due to the limited field of view of HR-pQCT compared CR and MRI.

Although characteristic of RA, cortical breaks indicating bone erosions are not specific to the disease; breaks also found in, for example, psoriatic arthritis, osteoarthritis, and crystal arthropathies. More important, cortical breaks are also found in HC. This represents a challenge regarding the clinical significance and nature of a cortical break as seen on HR-pQCT. While in CR the terms bone erosion and cortical break are used interchangeably, this should not be the case for cortical breaks seen with HR-pQCT. Indeed, some cortical breaks on HR-pQCT suggest a vascular channel or a bone cyst not related to RA. In HC, cortical breaks can reflect damage as a result of natural bone turnover or a microdamage repair process ${ }^{24,25}$. In addition to cortical erosions, which are full cortical breaks, intracortical bone porosity without erosions can also be found in RA, increasing the complexity of cortical bone changes in RA ${ }^{26}$. SPECTRA developed preliminary definitions for erosions, vascular channels, and subchondral cysts, which need validation. Methods of validation will be the comparison with microCT and histology in cadaver fingers. Similar to the detailed scoring of CR, interpreting bone changes on HR-pQCT will require training7. Quantification of these cortical and trabecular bone changes will need new imaging analysis systems. It is highly desirable that a (semi)automatic algorithm be developed in view of the high amount of image slices for increasing feasibility in research and practice. It will be necessary to investigate the relationship between the bone changes found on HR-pQCT and other imaging techniques, both cross-sectionally and longitudinally. For example, bone damage in RA reflects a history of events that are driven by inflammatory mechanisms, which can be visualised by MRI and ultrasound (e.g., bone marrow edema, synovitis, enthesitis, tendinitis, vascularisation) ${ }^{4}$. It is of interest to determine whether the bone changes visualised by HR-pQCT follow this inflammatory process, at which locations, and in which order. Finally, it needs to be investigated whether the heterogeneity seen in cortical breaks potentially can be used to better understand the pathophysiology of bone damage, and the predictive value of certain lesions for progression in damage. SPECTRA will continue to work on refining definitions for cortical breaks and erosions, and develop definitions for other abnormalities found in cortical bone and trabecular bone structures imaged by HR-pQCT. SPECTRA initiated studies comparing the cortical and trabecular bone defects imaged by HR-pQCT with conventional radiology, histology, microCT, MRI, and ultrasound in order to validate the HR-pQCT as a tool for measuring outcome in rheumatologic clinical trials. Moreover, SPECTRA aims at providing a substantial methodological basis by the filters of truth, discrimination and feasibility as described by the Outcome Measures in Rheumatology (OMERACT) Filters 1.0 and 2.0 for application as an OMERACT Special Interest Group ${ }^{27}$.

This image review demonstrates HR-pQCT images of MCP joints showing the cortical and subchondral trabecular bone structure in $\mathrm{HC}$ and the heterogeneous spectrum of cortical breaks in patients with RA. Future studies are needed to validate the HR-pQCT and to investigate its application in research and practice. 


\section{References}

1 Schett, G. \& Gravallese, E. Bone erosion in rheumatoid arthritis: mechanisms, diagnosis and treatment. Nat Rev Rheumatol 8, 656-664 (2012).

2 Pettit, A. R., Walsh, N. C., Manning, C., Goldring, S. R. \& Gravallese, E. M. RANKL protein is expressed at the pannus-bone interface at sites of articular bone erosion in rheumatoid arthritis. Rheumatology (Oxford) 45, 1068-1076 (2006).

$3 \quad$ Kleyer, A. et al. Bone loss before the clinical onset of rheumatoid arthritis in subjects with anticitrullinated protein antibodies. Ann Rheum Dis (2013).

Sommer, O. J. et al. Rheumatoid arthritis: a practical guide to state-of-the-art imaging, image interpretation, and clinical implications. Radiographics 25, $381-398$ (2005).

Lillegraven, S., van der Heijde, D., Uhlig, T., Kvien, T. K. \& Haavardsholm, E. A. What is the clinical relevance of erosions and joint space narrowing in RA? Nat Rev Rheumato/ 8, 117-120 (2012).

Gravallese, E. M. \& Walsh, N. C. Rheumatoid arthritis: Repair of erosion in RA-shifting the balance to formation. Nat Rev Rheumatol 7, 626-628 (2011).

Boini, S. \& Guillemin, F. Radiographic scoring methods as outcome measures in rheumatoid arthritis: properties and advantages. Ann Rheum Dis 60, 817-827 (2001). simplification of the Sharp/van der Heijde radiological assessment in rheumatoid arthritis. Rheumatology (Oxford) 38, 941-947 (1999).

Ravindran, V. \& Rachapalli, S. An overview of commonly used radiographic scoring methods in rheumatoid arthritis clinical trials. Clin Rheumato/ 30, 1-6 (2011).

Rev Rheumatol 10, 304-313 (2014).

11 Cheung, A. M. et al. High-resolution peripheral quantitative computed tomography for the assessment of bone strength and structure: a review by the Canadian Bone Strength Working Group. Curr Osteoporos Rep 11, 136-146 (2013).

Stach, C. M. et al. Periarticular bone structure in rheumatoid arthritis patients and healthy individuals assessed by high-resolution computed tomography. Arthritis Rheum 62, 330-339 (2010).

Finzel, S. et al. Repair of bone erosions in rheumatoid arthritis treated with tumour necrosis factor inhibitors is based on bone apposition at the base of the erosion. Ann Rheum Dis 70, 1587-1593 (2011).

Fouque-Aubert, A. et al. Assessment of hand bone loss in rheumatoid arthritis by high-resolution peripheral quantitative CT. Ann Rheum Dis 69, 1671-1676 (2010).

Barnabe, C. \& Feehan, L. High-resolution peripheral quantitative computed tomography imaging protocol for metacarpophalangeal joints in inflammatory arthritis: the SPECTRA collaboration. J Rheumatol 39, 1494-1495 (2012).

Aletaha, D. et al. 2010 Rheumatoid arthritis classification criteria: an American College of Rheumatology/ European League Against Rheumatism collaborative initiative. Arthritis and rheumatism 62, 2569-2581 (2010).

Boers, M., Jacobs, J. W., van Vliet Vlieland, T. P. \& van Riel, P. L. Consensus Dutch health assessment questionnaire. Ann Rheum Dis 66, 132-133 (2007).

Topfer, D., Finzel, S., Museyko, O., Schett, G. \& Engelke, K. Segmentation and quantification of bone erosions in high-resolution peripheral quantitative computed tomography datasets of the metacarpophalangeal joints of patients with rheumatoid arthritis. Rheumatology (Oxford) 53, 65-71 (2014).

Barnabe, C., Szabo, E., Martin, L., Boyd, S. K. \& Barr, S. G. Quantification of small joint space width, periarticular bone microstructure and erosions using high-resolution peripheral quantitative computed tomography in rheumatoid arthritis. Clin Exp Rheumatol 31, 243-250 (2013). define predictive factors for the topography of periarticular erosion formation in inflammatory arthritis. Arthritis Rheum 60, 1042-1051 (2009).

21 Hecht, C. et al. Additive effect of anti-citrullinated protein antibodies and rheumatoid factor on bone erosions in patients with RA. Ann Rheum Dis (2014).

Lee, C. H. et al. Correlation of structural abnormalities of the wrist and metacarpophalangeal joints evaluated by high-resolution peripheral quantitative computed tomography, 3 Tesla magnetic resonance imaging and conventional radiographs in rheumatoid arthritis. Int J Rheum Dis (2014).

Geusens, P. P. \& Finzel, S. Imaging: Bone erosions in rheumatoid arthritis: better to see more? Nat Rev Rheumato/ 9, 385-386 (2013).

Ejbjerg, B. et al. Magnetic resonance imaging of wrist and finger joints in healthy subjects occasionally shows changes resembling erosions and synovitis as seen in rheumatoid arthritis. Arthritis Rheum 50, 1097 1106 (2004).

Zhu, T. Y. et al. Structure and strength of the distal radius in female patients with rheumatoid arthritis: a case-control study. J Bone Miner Res 28, 794-806 (2013).

Boers, M., Kirwan, J. R. \& Tugwell, P. The OMERACT Handbook. (2015). 


\section{Supplementary files}
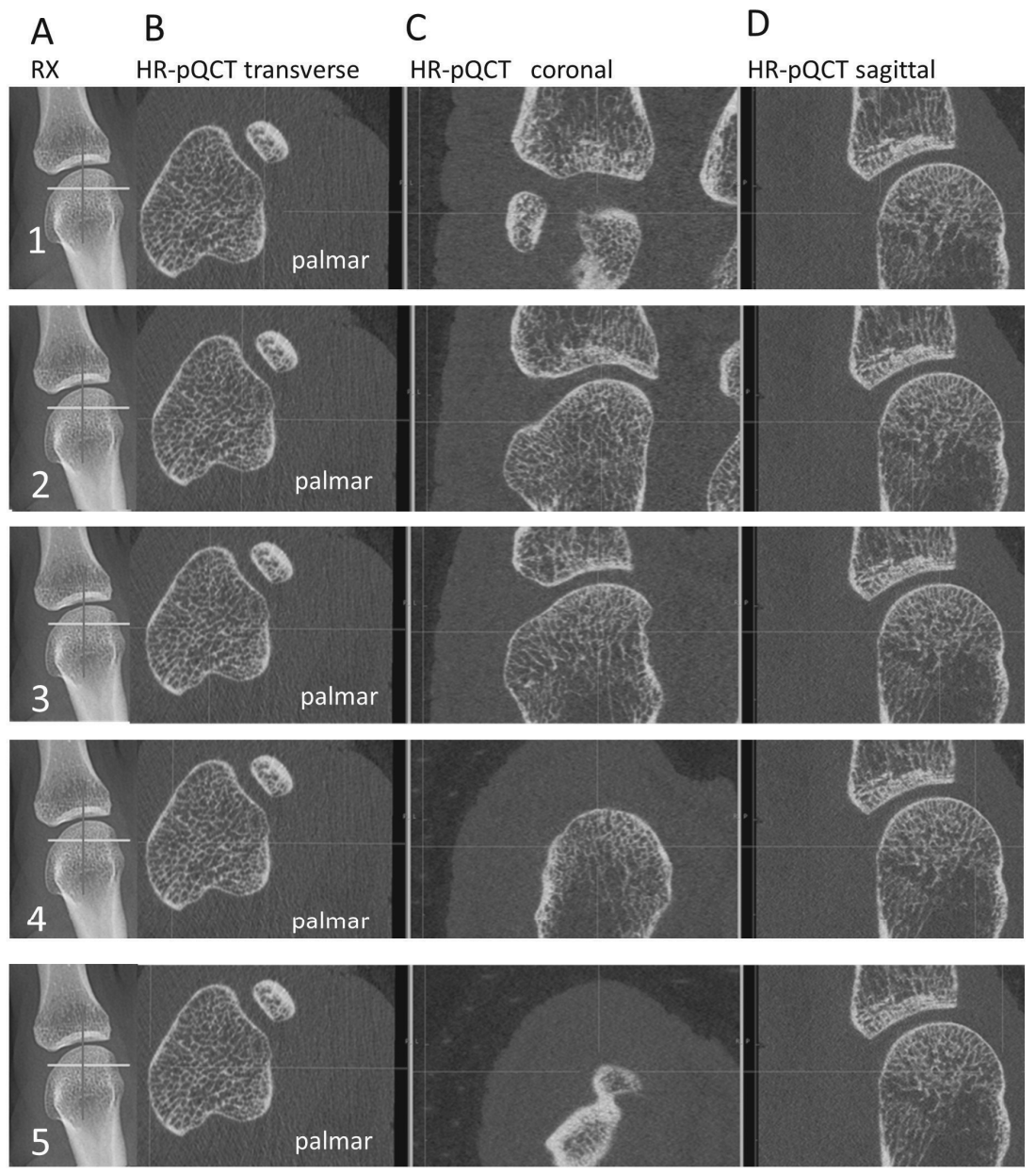

Supplementary Figure 2.1. An overview of an metacarpophalangeal joint with normal cortex viewed by a radiograph $(\mathrm{A})$ and high-resolution peripheral quantitative computed tomographic imaging at different positions; with the cross locator in the same slice of the same joint in transverse (B), coronal (C) and sagittal (D) views moving from palmar to dorsal side (panel 1, 2, 3, 4 and 5). Yellow line corresponds with the transversal position within the joint. The blue line corresponds with the movement of the green cross locator from palmar to dorsal side. 

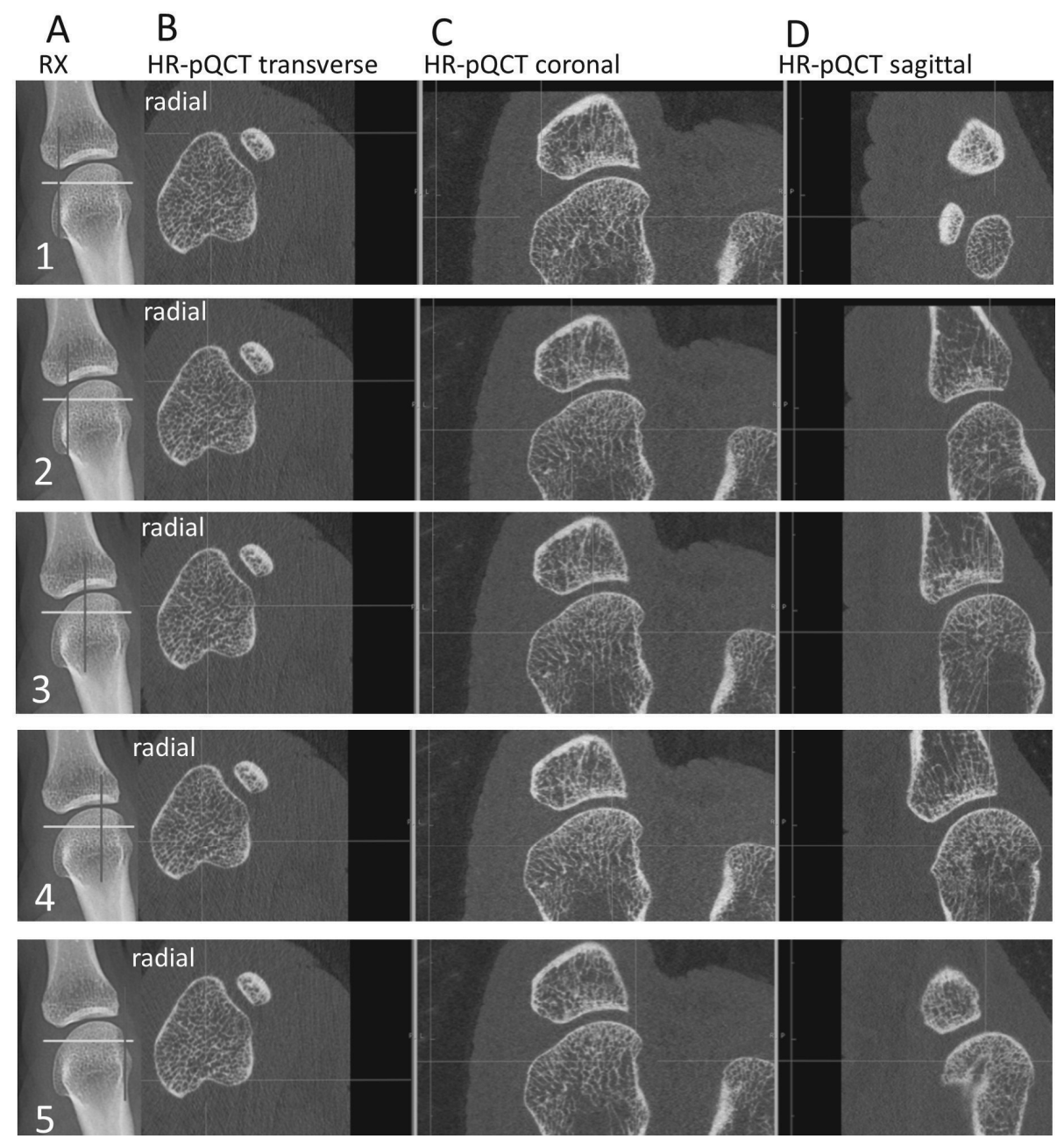

Supplementary Figure 2.2. An overview of an MCP joint with normal cortex viewed by a radiograph (A) and HR-pQCT imaging at different positions with the cross locator in the same slice of the same joint in transverse (B), coronal (C) and sagittal (D) views moving from radial to ulnar side (panel 1, 2, 3, 4 and 5). Yellow line corresponds with the transversal position within the joint. The blue line corresponds with the movement of the green cross locator from radial to ulnar side. 


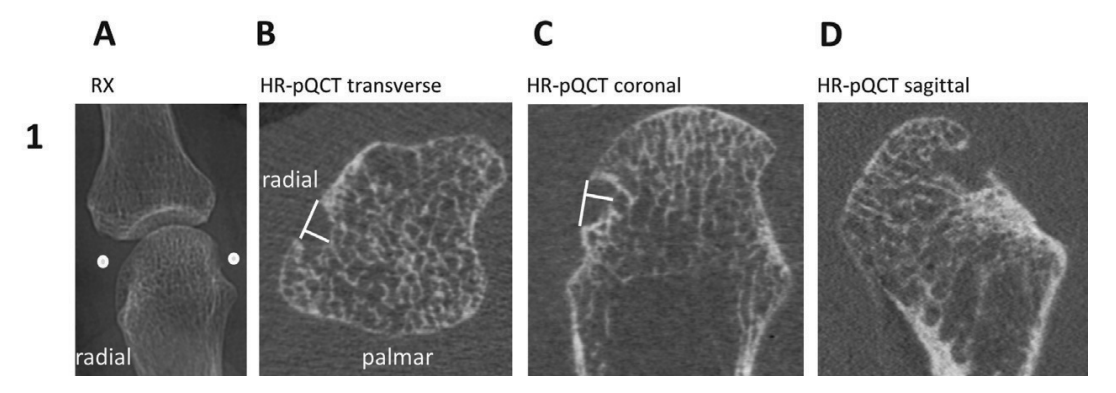

Supplementary Figure 2.3. An overview of an MCP joint viewed by a radiograph (A) and HR-pQCT imaging in transverse, coronal and sagittal views $(B, C, D)$. Measuring maximal width (white line) and depth (red line) of a cortical break in an MCP joint on HR-pQCT image in Osirix in a transverse and coronal plane. Width and depth are measured in transverse and coronal or sagittal plane. Lines need to be perpendicular to each other.

Supplementary Figure 2.4 and Figure 2.5. Three dimensional animation of an MCP joint are shown. Supplementary Figure 4 shows a normal joint.

http://www.jrheum.org/content/jrheum/

suppl/2016/09/13/43.10.1914.DC1/160646DataSuppl2.mp4

Supplementary Figure 5 shows a joint of an RA patient with cortical breaks compatible with bone erosions.

http://www.jrheum.org/content/irheum/

suppl/2016/09/13/43.10.1914.DC 1/160646DataSuppl3.mp4 
Visual detection of cortical breaks in hand joints: reliability and validity of highresolution peripheral quantitative CT compared to microCT
A. Scharmga, M. Peters, A. van Tubergen,
J. van den Bergh, J. de Jong, D. Loeffen,
B. van Rietbergen, R. Weijers, P. Geusens

BMC Musculoskeletal Disord. 2016 Jul 11;17271 


\begin{abstract}
Background: To study the reliability and validity of high-resolution peripheral quantitative CT (HR-pQCT) with microCT ( $\mu C T)$ as gold standard in the visual detection of cortical breaks in metacarpophalangeal (MCP) and proximal interphalangeal (PIP) joints.
\end{abstract}

Methods: Ten cadaveric fingers (10 MCP and 9 PIP joints) were imaged by HR-pQCT and $\mu \mathrm{CT}$ and visually analyzed by two independent readers. Intra- and inter-reader reliability were evaluated for the presence (yes/no, kappa statistics) and the total number (intraclass correlation coefficient, ICC) of cortical breaks. Sensitivity, specificity, positive and negative predictive value (PPV respectively NPV) of HR-pQCT in detecting cortical breaks were calculated.

Results: With HR-pQCT, mean 149 cortical breaks were identified and with $\mu C T$ mean 129 ( $p<0.05)$. Intra-reader reliability for the presence of a cortical break per quadrant was $0.52(95 \% \mathrm{Cl} 0.48-0.56)$ and 0.71 (95\% Cl 0.67-0.75) for HR-pQCT and $\mu C T$, respectively, and for the total number of cortical breaks 0.61 (95\% $\mathrm{Cl} 0.49-0.70)$ and $0.75(95 \% \mathrm{Cl} 0.68-0.82)$. Inter-reader reliability for the presence of a cortical break per quadrant was $0.37(95 \% \mathrm{Cl} 0.33-0.41)$ and $0.45(95 \% \mathrm{Cl} 0.41-0.49)$ for HRpQCT and $\mu \mathrm{CT}$, respectively, and for the number of cortical breaks $0.55195 \% \mathrm{Cl} 0.43$ $0.65)$ and $0.54(95 \% \mathrm{Cl} 0.35-0.67)$. Sensitivity, specificity, PPV and NPV of HR-pQCT were $81.6 \%, 64.0 \%, 81.6 \%$, and $64.0 \%$ respectively.

Conclusion: Cortical breaks were commonly visualized in MCP and PIP joints with HRpQCT and $\mu C T$. Reliability of both HR-pQCT and $\mu C T$ was fair to moderate. HR-pQCT was highly sensitive to detect cortical breaks with $\mu \mathrm{CT}$ as gold standard. 


\section{Introduction}

Peri-articular cortical breaks are one of the characteristic features of bone involvement in rheumatoid arthritis (RA) and predictors of further radiographic progression ${ }^{1,2}$. Early detection of cortical breaks is an important indicator for intensifying treatment in order to modify the disease course ${ }^{3}$. In daily clinic, conventional radiographs (CR) are considered the gold standard for detection of cortical breaks in the hand joints in rheumatic diseases. CR is widely available, fast to perform, relatively cheap, and extensively validated, however its sensitivity to detect structural bone changes is low compared to computed tomography (CT), MRI and ultrasound ${ }^{4 \cdot 7}$. A novel, sensitive imaging technique is High-Resolution peripheral Quantitative Computed Tomography (HR-pQCT) ${ }^{8}$. HR-pQCT allows analysis of the cortical and trabecular microarchitecture of peripheral bones with an isotropic resolution of 82 micrometer $(\mu \mathrm{m})$. This technique is now also applied for 3-D assessment of the bone microarchitecture in the hand joints ${ }^{8-10}$. A study by Stach et al. has demonstrated that HR-pQCT is more sensitive than CR in detecting cortical breaks in the hand joints in RA and also in healthy controls ${ }^{8}$. However, the resolution of the HR-pQCT images can be of the same order as the thickness of the cortical bone in finger joints. Due to partial volume effects, it is possible that thin cortices are falsely identified as breaks. Therefore, in particular with thin cortices, the reliability, sensitivity and specificity of the measurements might be impaired and depend on the reader's perception.

The aims of this study were 1). to investigate the intra- and inter-reader reliability, and 2 ). to determine the sensitivity, specificity, positive predictive value (PPV) and negative predictive value (NPV) of HR-pQCT in detecting cortical breaks in hand joints, using $\mu C T$ images with a much higher resolution $(18 \mu \mathrm{m})$ as gold standard. We were particularly interested in the methodology of identifying cortical breaks by HR-pQCT, not to study the clinical value of these cortical breaks. We hypothesized that HR-pQCT is a reliable and sensitive imaging method for identifying cortical breaks in hand joints compared to $\mu \mathrm{CT}$.

\section{Methods}

\section{Specimens}

For this study, we used cadaveric specimen, because $\mu \mathrm{CT}$ imaging can only be executed in-vitro. MCP and PIP joints of ten female right hand human cadaveric index fingers were imaged by both HR-pQCT and $\mu \mathrm{CT}$. The donors had dedicated their body by testament signed during life to the Department of Anatomy and Embryology of the University of Amsterdam, the Netherlands. The fingers were fixated in formalin.

\section{$H R-p Q C T$ and $\mu C T$ image acquisition}

HR-pQCT (XtremeCT1, Scanco Medical AG, Switzerland) scans were performed at clinical in vivo settings, i.e. at $60 \mathrm{kVp}$ tube voltage, $900 \mu \mathrm{A}$ tube current, $100 \mathrm{~ms}$ integration time and $82 \mu \mathrm{m}$ voxel size. $\mu \mathrm{CT}$ ( $\mu \mathrm{CT}$ 80, Scanco Medical AG, Switzerland) scans were performed at $70 \mathrm{kVp}$ tube voltage, $114 \mu \mathrm{A}$ tube current, 300 
ms integration time and $18 \mu \mathrm{m}$ voxel size. On HR-pQCT, the region of interest of the MCP joint covered an area of $18.04 \mathrm{~mm}, 220$ slices and for the PIP joint $9.02 \mathrm{~mm}$, 110 slices.

On $\mu C T$, the region of interest covered an area of $15.26 \mathrm{~mm} ; 848$ slices and for the PIP joint 9.45 mm, 525 slices (Supplementary Figure 3.1).

HR-pQCT and $\mu C T$ image analysis

Scans of HR-pQCT and $\mu C T$ were exported in Digital Imaging and Communications in Medicine (DICOM) format and analyzed using Osirix (v.5.8.5 64-bit) multiplanar DICOM viewer. Differences in the extent of the scanned areas as well as in joint angles were noticed because the fingers were scanned horizontally on HR-pQCT and vertically on $\mu \mathrm{CT}$. Corresponding first and last slices of the overlapping region were visually determined to ensure that the same region of interest was used in the detection of cortical breaks on both imaging modalities.

Two trained readers (AS and MP) independently scored the HR-pQCT and $\mu C T$ images visually for the presence of cortical breaks. The readers received extensive training from Study grouP for xtrEme Computed Tomography in Rheumatoid Arthritis (SPECTRA) and have additional reading experience, before the current dataset was read. Readers were aware of the hypothesis of this study. The images were not anonymized. HR-pQCT images were first and independently scored from $\mu$ CT images, with at least one day in between, and a two week interval for the rescoring by Reader 1 .

A cortical break was defined as a clear disruption of the cortex, seen on two consecutive slices on two orthogonal planes (on transverse and on sagittal or coronal plane) on HR-pQCT, and similarly, but on nine consecutive slices on $\mu C T$ to cover the same area as evaluated by HR-pQCT (Supplementary Figure 3.2).

To assess the location of the breaks in each joint, the transverse plane was divided into four quadrants: palmar, ulnar, dorsal and radial (Supplementary Figure 3.3). The phalangeal base and metacarpal head of the MCP joints, and proximal phalanx and distal phalanx of the PIP joints were separately assessed. In total eight quadrants per joint were analysed, four in the proximal bone, and four in the distal bone of the same joint. Each joint was systematically analysed per quadrant. Quadrants with large discrepancies between the readers (i.e. more than four breaks difference) were reexamined to identify reasons for discrepancy. Also, total volumetric bone mineral density (vBMD) of the specimens was calculated using HR-pQCT.

\section{Statistics}

Descriptive analyses were done to calculate the total number of cortical breaks scored by the readers per quadrant for each imaging modality.

The difference in the total number of cortical breaks detected with HR-pQCT versus $\mu C T$ was tested for statistical significance with Wilcoxon signed-rank test. Intra- and interreader reliability were calculated using Cohen's Kappa ( $\mathrm{K}$ ) and intraclass correlations coefficient (ICC) with a two-way random model and absolute agreement. $\mathrm{k}$ value was 
calculated for the presence (yes/no) of a cortical break per quadrant and ICC values were calculated for the total number of cortical breaks per quadrant. $\mathrm{K}$ and ICC were calculated on the level of all available quadrants. Kappa values were also recalculated, corrected for potential prevalence and bias within the kappa value (Prevalence-Adjusted Bias Adjusted Kappa, PABAK) ${ }^{11,12}$. Reliability was rated according to Landis et al.: $<0.00$ poor, 0.00-0.20 slight, 0.21-0.40 fair, 0.41-0.60 moderate, 0.61-0.80 substantial, 0.81-1.00 almost perfect ${ }^{13}$. Sensitivity, specificity, PPV, and NPV of HR-pQCT in the detection of cortical breaks were calculated with $\mu C T$ as gold standard. The mean value of the two readings of reader 1 (AS) was used for this purpose. Statistical analyses were performed with SPSS Statistics for Windows version 23.0 (IBM Corp., Armonk, NY).

\section{Results}

The mean age of the donors was $85.1 \pm 9.6$ years, the medical history was unknown. Average $\mathrm{vBMD}$ was $203 \mathrm{mgHA} / \mathrm{cm}^{3}$ for $\mathrm{MCP}$ joints, $293 \mathrm{mgHA} / \mathrm{cm}^{3}$ for PIP joints and $245 \mathrm{mgHA} / \mathrm{cm}^{3}$ for the total joints. The scans of ten MCP and nine PIP joints with in total 152 quadrants were available for analysis. One PIP joint could not be evaluated due to a missing $\mu \mathrm{CT}$ scan. Furthermore, Reader 1 considered the quality of the images of the metacarpal head in one MCP joint as too low on $\mu \mathrm{CT}$ due to a protocol error during scanning. Therefore four quadrants were excluded in the analyses of the $\mu C T$ images. Table 3.1 shows the total number of cortical breaks each reader found on HRpQCT and $\mu C T$ images. The differences in scores between the first and second reading of Reader 1 were not statistically significant on HR-pQCT (139 vs 118 breaks, $p=.064$ ) and $\mu \mathrm{CT}$ (142 vs 156 breaks, $p=.163$ ). However, the difference in the mean score between HR-pQCT versus $\mu C T$ was statistically significant (respectively, 129 and 149 breaks, $p<0.05)$. The total number of cortical breaks on HR-pQCT scored by Reader 1 (first reading) versus Reader 2 was not statistically significant (respectively, 139 versus 151 breaks, $p=.288$ ). On $\mu \mathrm{CT}$, Reader 2 found significantly more breaks than Reader 1 (first reading) (241 vs 142 breaks, $p<.001$ ).

Table 3.1. Number of cortical breaks scored per imaging modality

\begin{tabular}{llll}
\hline & HR-pQCT & $\mu C T$ & $p$ value \\
\hline $\begin{array}{l}\text { Reader 1 (first reading) } \\
\text { Reader 1 (second reading) }\end{array}$ & $139(7.3 \pm 4.1)$ & $142(7.4 \pm 4.0)$ & $\mathrm{p}=.562$ \\
& $118(6.2 \pm 2.6)$ & $156(8.2 \pm 3.6)$ & $\mathrm{p}<.000$ \\
Mean score Reader 1 & $129(6.7 \pm 3.0)$ & $149(7.8 \pm 3.6)$ & $\mathrm{p}=.018$ \\
& $151(7.9 \pm 3.3)$ & $241(12.6 \pm 6.3)$ & $\mathrm{p}<.000$ \\
Reader 2 & & & \\
\hline
\end{tabular}

In total 4 quadrants with large discrepancies between the readers were re-examined. Several reasons for discrepancy were identified: 
On $\mu \mathrm{CT}$ we defined a cortical break when present on nine consecutive slices. Sometimes Reader 1 observed the cortical break on eight consecutive slices, hence not considering it a break, whereas Reader 2 observed it on nine consecutive slices, thereby fulfilling the criteria for a break.

- The smaller the break, the less agreement between the readers. An example of this discrepancy is shown in Supplementary figure 3.4 panel A and B.

- Due to the low bone mineral density and thin cortices, there was low contrast in some cases (example in Supplementary figure 3.4 panel C).

- Reader 1 considered a break as one large break, whereas Reader 2 counted several small cortical breaks inside the same large break lexample in Supplementary figure 3.4 panel D).

Table 3.2 shows the intra- and inter-reader reliability based on the 152 quadrants on HR-pQCT and 148 quadrants on $\mu C T$ that were evaluated. Intra-reader reliability was moderate to substantial for the presence of breaks (HR-pQCT: $\mathrm{k}=0.52$ and $\mu C T: \mathrm{k}=0.71$ ) and for the number of breaks (HR-pQCT: ICC $=0.61$ and $\mu C T$ : ICC =0.75).

Inter-reader reliability was fair to moderate for the presence of breaks (HR-pQCT:K= 0.37 and $\mu C T: \mathrm{K}=0.45$ ) and for the number of breaks (HR-pQCT: ICC $=0.55$ and $\mu C T$ : $I C C=0.54$ ). The values of PABAK were comparable (Table 3.2).

Sensitivity, specificity, PPV and NPV of HR-pQCT in the detection of cortical breaks with $\mu C T$ as gold standard were calculated with the mean scores of Reader 1 (reading 1 and 2) and the score of Reader 2. The sensitivity was $81.6 \%$, specificity $64 \%$, PPV $81.6 \%$, and NPV $64 \%$ respectively for Reader 1, and sensitivity $68.9 \%$, specificity $69.4 \%$, PPV $82.6 \%$ and NPV $51.5 \%$ for Reader 2 (Table 3.3 and supplementary file 3.5).

In Figure 3.1, several examples of cortical breaks on corresponding HR-pQCT and $\mu C T$ images are presented. Panel $A$ and $B$ show a cortical break on both HR-pQCT and $\mu \mathrm{CT}$. In panel C, a discontinuity of the cortex is found on HR-pQCT. However, it did not meet the definition of a cortical break applied in this study, because it was visible on one slice only, leading to discrepancy with the results from $\mu C T$. In panel $D$, a cortical break was detected on HR-pQCT, but not on $\mu C T$, where a thin cortical lining was seen. 

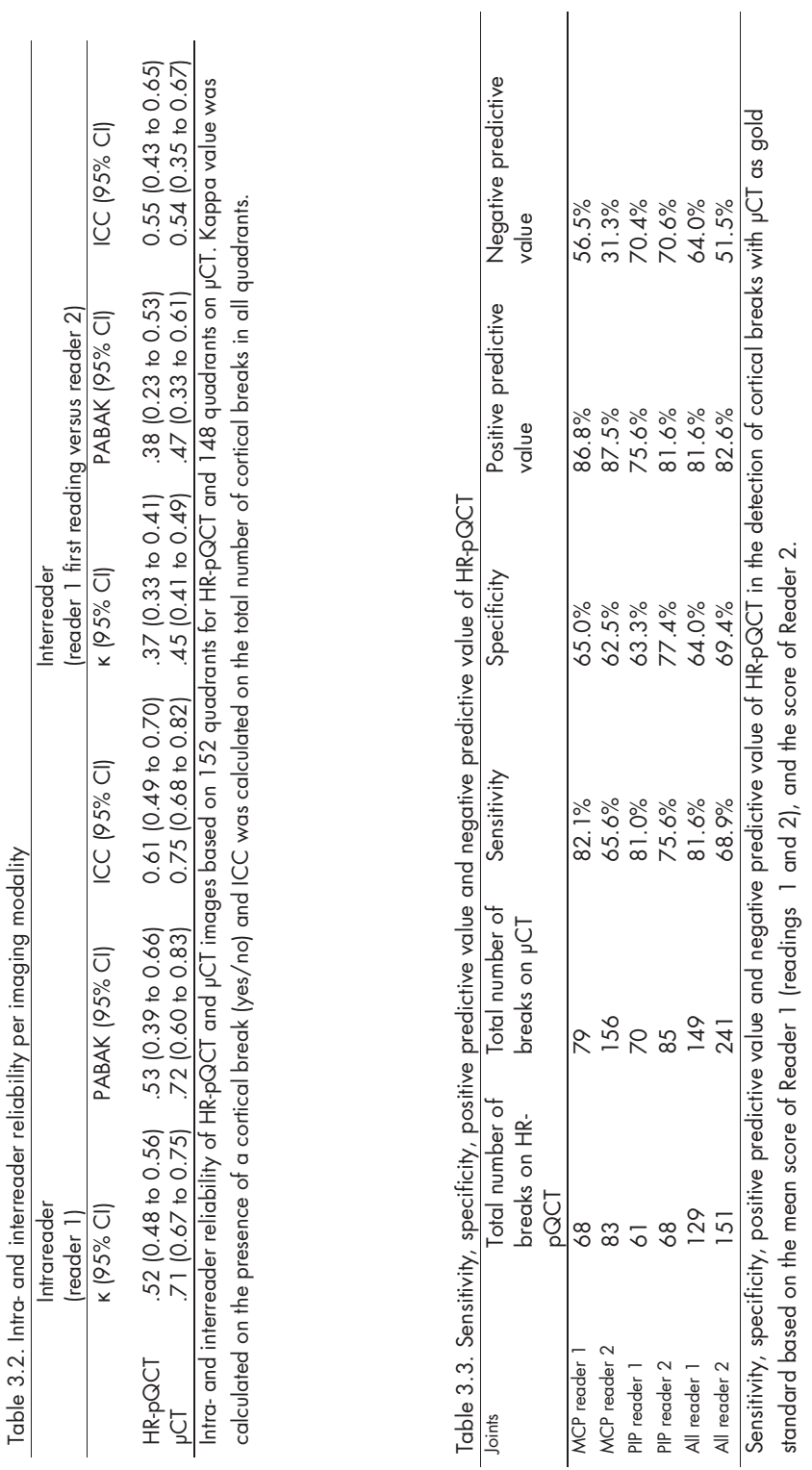


\section{HR-pQCT}

A

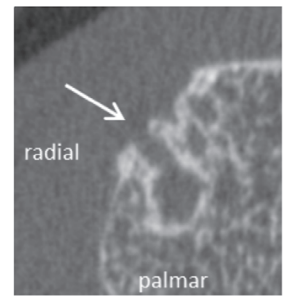

B

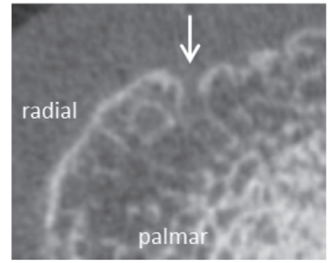

C

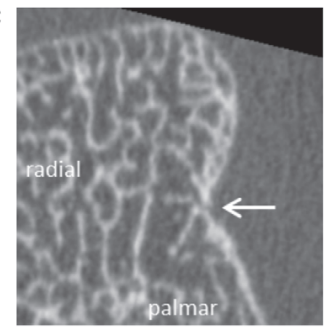

D

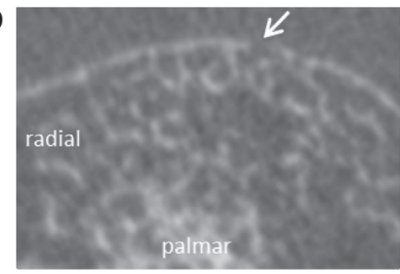

microCT
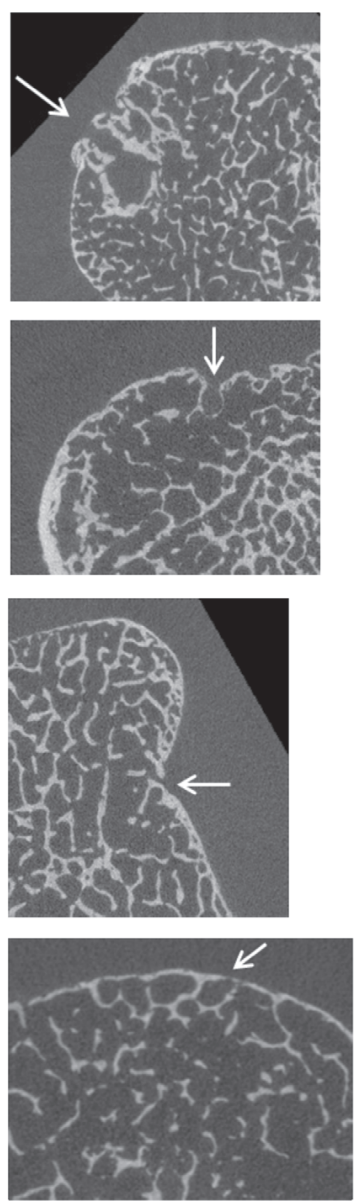

Figure 3.1. Corresponding images of cortical breaks on HR-pQCT and $\mu C T$. Panel a A discontinuity of the cortex (arrow) meeting the definition of a cortical break was seen on both HR-pQCT and $\mu C T$. Panel $b$ A discontinuity of the cortex (arrow) meeting the definition of a cortical break was seen on both HR$p Q C T$ and $\mu C T$. Panel $c$ A discontinuity of the cortex (arrow) is seen on one slice only on HR- $p Q C T$, thereby not fulfilling the definition for a break on HR-pQCT. A clear cortical break (arrow) is seen on $\mu C T$, which was also seen on nine subsequent slices, thereby fulfilling the definition of a break. Panel $d$ A discontinuity of the cortex (arrow) meeting the definition of a cortical break is seen on HR-pQCT. On $\mu C T$, the cortical lining is intact (arrow). Abbreviations: MCP; metacarpophalangeal, HR-pQCT; highresolution peripheral quantitative computed tomography, $\mu \mathrm{CT}$; microCT 


\section{Discussion}

This study is the first that reports on aspects of reliability and validity of detecting cortical breaks in hand joints using HR-pQCT with $\mu C T$ as gold standard. Cortical breaks were found in all joints with both imaging modalities. Intrareader reliability of HR-pQCT and $\mu C T$ was moderate to substantial, while interreader reliability was fair to moderate. The sensitivity of HR-pQCT in detecting cortical breaks was high (81.6\%). In our study, only cortical discontinuities meeting the definition of a break, i.e. clearly visible cortical interruptions on at least $2 \mathrm{HR}$-pQCT (or $9 \mu \mathrm{CT}$ ) consecutive slices in 2 planes, were scored as a cortical break. They may have different pathological or physiological backgrounds, such as erosions or vascular channels 8,9 . A formal classification system for defining breaks visualized on HR-pQCT and $\mu C T$ is lacking. Histological examination is needed to provide more insight in the nature of these cortical breaks and for developing definitions.

In a previous study by Stach et al. an almost perfect and substantial intra- and interreader reliability was reported using HR-pQCT for grading bone lesions and discrimination between healthy individuals and RA patients $\mathrm{K}=0.82$ and $\mathrm{K}=0.75$ respectively), but reliability on the presence and number of cortical breaks was not reported $^{8}$. The precision in scoring abnormalities visualized with several imaging techniques varies widely, even by experienced readers, as has been demonstrated for example for scoring radiographs in RA (ICC ranged from 0.65 to 0.99$)^{14}$. In general, lower values for inter-reader reliability in comparison with intra-reader reliability are reported ${ }^{15}$, corresponding to our findings. In our study, the breaks were scored visually, which is reader dependent. An automatic scoring algorithm, with detection of predefined definitions of breaks and executed automatically by the computer, could potentially improve reliability by minimizing reader interventions.

We investigated the sensitivity of HR-pQCT in detecting cortical breaks with $\mu C T$ as the gold standard and found a high sensitivity (81.6\%). Unfortunately, no comparative studies are available. In contrast, two studies used HR-pQCT as the reference method for investigating the sensitivity to detect cortical breaks of other imaging modalities 9,16 . These studies reported a sensitivity of $85.7 \%$ for MRI, $60.9 \%$ for CR, and $83-100 \%$ for ultrasound with HR-pQCT as the reference method 9,16 . We found a lower specificity of HR-pQCT in detecting cortical breaks $(64 \%)$ in comparison to sensitivity. A possible explanation for this could be a phenomenon attributed to a partial volume effect leading to a reduced cortical signal on HR-pQCT, giving the impression that a cortical break is present, whereas on $\mu C T$ the cortex is intact. An example of this is shown in Figure 3.1, panel D.

There are several limitations of this study. First, we evaluated whether the total number of breaks counted per quadrant corresponded between the two imaging modalities, but did not consider correspondence in exactly the same location. This might have led to an overestimation of the reliability. Second, we used fingers from cadaver specimens with unknown medical history and a relatively high mean age (85.1 years). Due to the old age of the donors, and the preservation in formalin, the cortices might become less 
mineralized ${ }^{17}$. The average $v B M D$ of the specimens was $245 \mathrm{mgHA} / \mathrm{cm}^{3}$, which is some $20 \%$ lower than the average in the normal population $\left(>300 \mathrm{mgHA} / \mathrm{cm}^{3}\right)^{10,18}$. This may hamper the scoring of a cortical break on HR-pQCT.

It is also possible that thin regions were falsely identified as a cortical break. However, the use of cadaveric specimens was essential as in vivo human subjects cannot be measured by $\mu \mathrm{CT}$ because of a long scanning time. Third, the cadaveric specimens had slightly different orientations in the HR-pQCT versus the $\mu \mathrm{CT}$ scanner. Despite the careful visual matching of the regions of interest on HR-pQCT and $\mu C T$, the angle at which the transversal images were viewed was slightly different in some joints and a cortical break might therefore be missed.

Fourth, a discrepancy between the readers regarding the number of cortical breaks identified on $\mu \mathrm{CT}$ was noticed. $\mu \mathrm{CT}$ images provide much detail, and in particular very small cortical interruptions were not always picked up by Reader 1. This indicates that, when visually analyzing $\mu \mathrm{CT}$ images, more stringent definitions are necessary than when using HR-pQCT because of the higher resolution.

\section{Conclusions}

Cortical breaks were commonly visualized in hand joints with HR-pQCT and $\mu \mathrm{CT}$. Reliability of both HR-pQCT and $\mu C T$ was fair to moderate. HR-pQCT was sensitive to detect cortical breaks with $\mu \mathrm{CT}$ as gold standard. In spite of the limitations of our study, including the discrepancy of $\mu \mathrm{CT}$ results between the readers, we have shown that HRpQCT is highly sensitive to detect cortical breaks with a fair to moderate reliability compared to $\mu \mathrm{CT}$. Our findings need further evaluation, preferably with focus on histological analyses to clarify the nature of the breaks and to establish more reliable definitions and a classification system for analyzing cortical breaks on high-resolution CT images. 


\section{References}

1 Schett, G. \& Gravallese, E. Bone erosion in rheumatoid arthritis: mechanisms, diagnosis and treatment. Nat Rev Rheumatol 8, 656-664 (2012).

2 Geusens, P. \& van den Bergh, J. Bone erosions in rheumatoid arthritis. Rheumatology (Oxford) 53, 4-5 (2014).

Sommer, O. J. et al. Rheumatoid arthritis: a practical guide to state-of-the-art imaging, image interpretation, and clinical implications. Radiographics 25, 381-398 (2005).

Baillet, A. et al. Comparison of the efficacy of sonography, magnetic resonance imaging and conventional radiography for the detection of bone erosions in rheumatoid arthritis patients: a systematic review and meta-analysis. Rheumatology (Oxford) 50, 1137-1147 (2011).

Geusens, P. et al. High-resolution in vivo imaging of bone and joints: a window to microarchitecture. Nat Rev Rheumatol 10, 304-313 (2014).

Saraux, A. et al. Ability of the American College of Rheumatology 1987 criteria to predict rheumatoid arthritis in patients with early arthritis and classification of these patients two years later. Arthritis Rheum 44, 2485-2491 (2001).

Dohn, U. M. et al. Detection of bone erosions in rheumatoid arthritis wrist joints with magnetic resonance imaging, computed tomography and radiography. Arthritis Res Ther 10, R25 (2008).

Stach, C. M. et al. Periarticular bone structure in rheumatoid arthritis patients and healthy individuals assessed by high-resolution computed tomography. Arthritis Rheum 62, 330-339 (2010).

Finzel, S. et al. A detailed comparative study of high-resolution ultrasound and micro-computed tomography for detection of arthritic bone erosions. Arthritis Rheum 63, 1231-1236 (2011). Fouque-Aubert, A. et al. Assessment of hand bone loss in rheumatoid arthritis by high-resolution peripheral quantitative CT. Ann Rheum Dis 69, 1671-1676 (2010).

11 Sim, J. \& Wright, C. C. The kappa statistic in reliability studies: use, interpretation, and sample size requirements. Phys Ther 85, 257-268 (2005). Byrt, T., Bishop, J. \& Carlin, J. B. Bias, prevalence and kappa. J Clin Epidemio/ 46, 423-429 (1993). Landis, J. R. \& Koch, G. G. The measurement of observer agreement for categorical data. Biometrics 33, 159-174 (1977). experienced readers. J Rheumato/ 31 , 1062-1072 (2004).

Salaffi, F. \& Carotti, M. Interobserver variation in quantitative analysis of hand radiographs in rheumatoid arthritis: comparison of 3 different reading procedures. J Rheumato/ 24, 2055-2056 (1997).

Lee, C. H. et al. Correlation of structural abnormalities of the wrist and metacarpophalangeal joints evaluated by high-resolution peripheral quantitative computed tomography, 3 Tesla magnetic resonance imaging and conventional radiographs in rheumatoid arthritis. Int J Rheum Dis (2014).

Zebaze, R. M. et al. Intracortical remodelling and porosity in the distal radius and post-mortem femurs of women: a cross-sectional study. Lancet 375, 1729-1736 (2010).

18 Feehan, L., Buie, H., Li, L. \& McKay, H. A customized protocol to assess bone quality in the metacarpal head, metacarpal shaft and distal radius: a high resolution peripheral quantitative computed tomography precision study. BMC Musculoskelet Disord 14, 367 (2013). 


\section{Supplementary files}

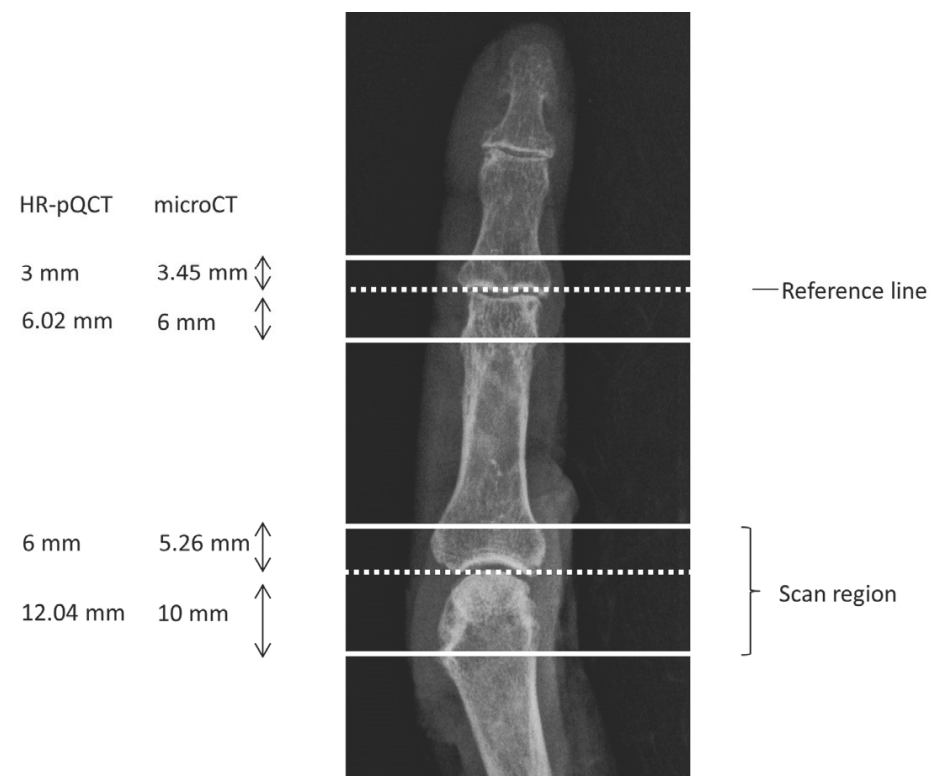

Supplementary figure 3.1. Method of selection of regions of interest in an MCP and PIP joint on HRpQCT and $\mu C T$. Total scan area for an MCP joint on HR-pQCT was $18.04 \mathrm{~mm}$ and for a PIP joint $9.02 \mathrm{~mm}$. Total scan area for an MCP joint on $\mu C T$ was $15.26 \mathrm{~mm}$ and for a PIP joint $9.45 \mathrm{~mm}$. HR$\mathrm{pQCT}$; high-resolution peripheral quantitative computed tomography, $\mu \mathrm{CT}$; micro computed tomography, MCP; metacarpophalangeal, PIP; proximal interphalangeal.

HR-pQCT $82 \mu \mathrm{m}$ per slice
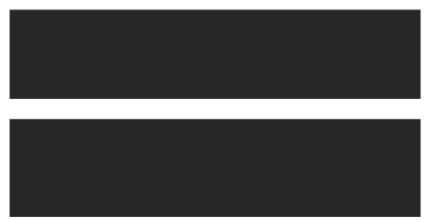

$\mu \mathrm{CT} 18 \mu \mathrm{m}$ per slice

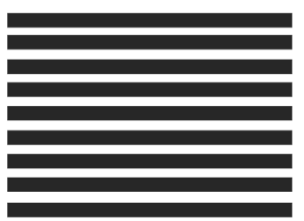

Supplementary figure 3.2. Resolution of HR-pQCT and $\mu \mathrm{CT}$ imaging. Resolution of HR-pQCT imaging is $82 \mu \mathrm{m}$, while the resoltuion of $\mu \mathrm{CT}$ imaging is $18 \mu \mathrm{m}$. To match both resolutions, two consecutive slices on HR-pQCT correspond with nine consecutive slices on $\mu C T$. HR-pQCT; high-resolution peripheral quantitative computed tomography, $\mu \mathrm{CT}$; micro $\mathrm{CT}$.

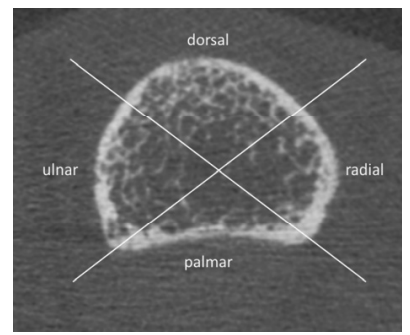

Supplementary figure 3.3. Division of a phalangeal base of an MCP joint. Phalangeal base is divided into palmar, ulnar, dorsal and radial quadrants. MCP: metacarpophalangeal. 
$\mu \mathrm{CT}$

A

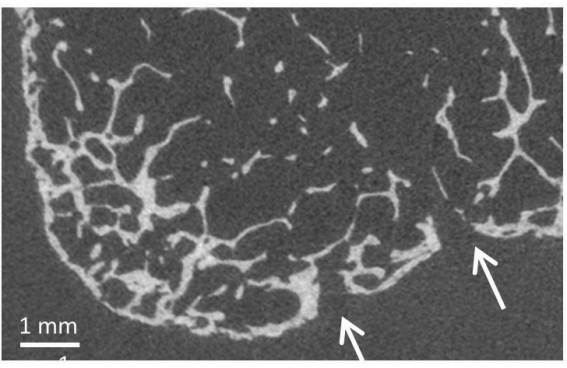

B

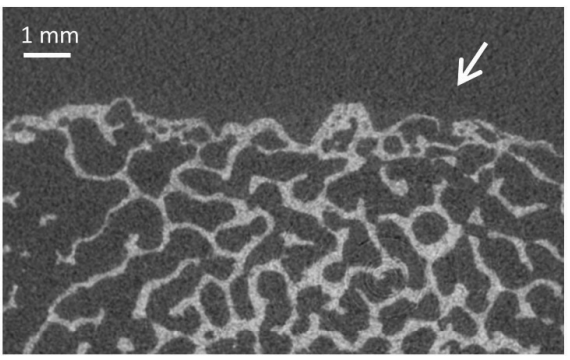

C

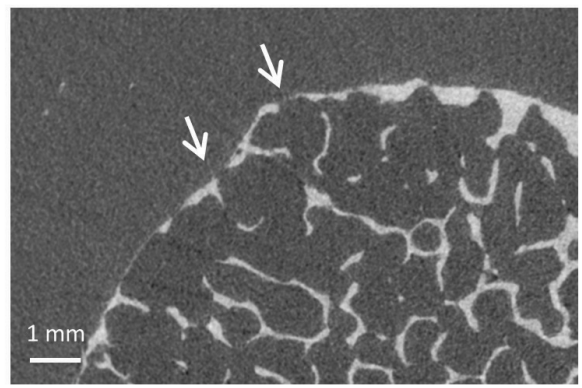

D

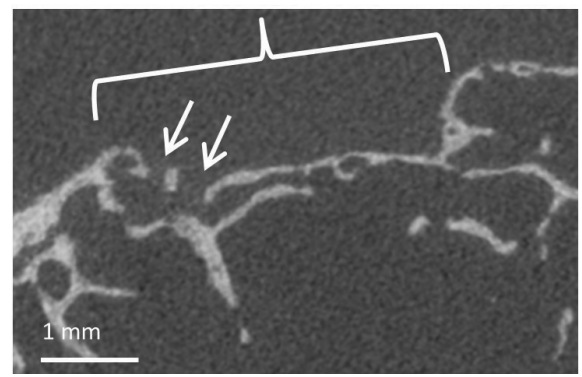

Supplementary figure 3.4. Examples of $\mu \mathrm{CT}$ images that could have attributed to the differences in scoring cortical breaks between Reader 1 and 2 .

Panel A. Large cortical breaks (arrow) show high agreement. Panel B. Small cortical breaks (arrow) show less agreement. Panel C. An extremely thin cortex (arrow). Panel D. One large break (in brackets) was counted by Reader 1, where Reader 2 considered this as several smaller cortical breaks (arrow). $\mu \mathrm{CT}$; microCT 
Table 3.1. $2 \times 2$ contingency table for Reader 1 all joints (MCP and PIP)

\begin{tabular}{l|lll}
\hline & uCT presence break & uCT absence break & Total \\
\hline HR-pQCT presence break & 80 & 18 & 98 \\
HR-pQCT absence break & 18 & 32 & 50 \\
Total & 98 & 50 & 148 quadrants \\
\hline
\end{tabular}

$2 \times 2$ table on the presence of a cortical break on $\mu$ CT (gold standard) and HR-pQCT of Reader 1 for all joints (MCP and PIP). Reader 1 considered 4 quadrants not evaluable due to the quality of the images due to a protocol error.

Table 3.2. $2 \times 2$ contingency table for Reader 1 for MCP joints

\begin{tabular}{l|lll}
\hline & uCT presence break & uCT absence break & Total \\
\hline HR-pQCT presence break & 46 & 7 & 53 \\
HR-pQCT absence break & 10 & 13 & 23 \\
Total & 56 & 20 & 76 quadrants \\
\hline
\end{tabular}

$2 \times 2$ table on the presence of a cortical break on $\mu$ CT (gold standard) and HR-pQCT of Reader 1 for MCP joints. Reader 1 considered 4 quadrants not evaluable due to the quality of the images due to a protocol error.

Table 3.3. $2 \times 2$ contingency table for Reader 1 for PIP joints

\begin{tabular}{l|lll}
\hline \multicolumn{1}{l}{ uCT presence break } & uCT absence break & Total \\
\hline HR-pQCT presence break & 34 & 11 & 45 \\
HR-pQCT absence break & 8 & 19 & 27 \\
Total & 42 & 30 & 72 quadrants \\
\hline
\end{tabular}

$2 \times 2$ table on the presence of a cortical break on $\mu \mathrm{CT}$ (gold standard) and HR-pQCT of Reader 1 for PIP joints. Reader 1 considered 4 quadrants not evaluable due to the quality of the images due to a protocol error.

Table 3.4. $2 \times 2$ contingency table for Reader 2 for all joints (MCP and PIP joints)

\begin{tabular}{|c|c|c|c|}
\hline & uCT presence break & uCT absence break & Total \\
\hline HR-pQCT presence break & 71 & 15 & 86 \\
\hline HR-pQCT absence break & 32 & 34 & 66 \\
\hline Total & 103 & 49 & 152 quadrants \\
\hline
\end{tabular}

$2 \times 2$ table on the presence of a cortical break on $\mu C T$ (gold standard) and HR-pQCT of Reader 2 for all joints (MCP and PIP).

Table 3.5. $2 \times 2$ contingency table for Reader 2 for $M C P$ joints

\begin{tabular}{l|lll}
\hline & uCT presence break & uCT absence break & Total \\
\hline HR-pQCT presence break & 42 & 6 & 48 \\
HR-pQCT absence break & 22 & 10 & 32 \\
Total & 64 & 16 & 80 quadrants \\
\hline
\end{tabular}

$2 \times 2$ table on the presence of a cortical break on $\mu C T$ (gold standard) and HR-pQCT of Reader 2 for MCP joints.

Table 3.6. $2 \times 2$ contingency table for Reader 2 for PIP joints

\begin{tabular}{|c|c|c|c|}
\hline & uCT presence break & uCT absence break & Total \\
\hline HR-pQCT presence break & 31 & 7 & 38 \\
\hline HR-pQCT absence break & 10 & 24 & 34 \\
\hline Total & 41 & 31 & 72 quadrants \\
\hline
\end{tabular}

$2 \times 2$ table on the presence of a cortical break on $\mu C T$ (gold standard) and HR-pQCT of Reader 2 for PIP joints.

Supplementary tables 3.5. $2 \times 2$ contingency tables for Reader 1 (all joints and MCP and PIP separately) and Reader 2 (all joints and MCP and PIP joints separately. 
Vascular channels in metacarpophalangeal
joints: a comparative histologic and high-
resolution imaging study

A. Scharmga, K.K. Keller, M. Peters,

A. van Tubergen, J. van den Bergh,

B. van Rietbergen, R. Weijers, D. Loeffen, E.M. Hauge, P. Geusens

Sci Rep. 2017 Aug 21;7(1) 


\begin{abstract}
We evaluated whether cortical interruptions classified as vascular channel (VC) on highresolution peripheral quantitative computed tomography (HR-pQCT) could be confirmed by histology. We subsequently evaluated the image characteristics of histologically identified VCs on matched single and multiplane HR-pQCT images. Four 3-mm thick portions in three anatomic metacarpophalangeal joint specimens were selected for histologic sectioning. First, VCs identified with HR-pQCT were examined for confirmation on histology. Second and independently, VCs identified by histology were matched to single and multiplane HR-pQCT images to assess for presence of cortical interruptions. Only one out of five cortical interruptions suggestive for VC on HR-pQCT could be confirmed on histology. In contrast, 52 VCs were identified by histology of which $39(75 \%)$ could be classified as cortical interruption or periosteal excavation on matched single HR-pQCT slices. On multiplane HR-pQCT images, 11 (21\%) showed a cortical interruption in at least two consecutive slices in two planes, $36(69 \%)$ in at least one slice in two planes and five $(10 \%)$ showed no cortical interruption. Substantially more VCs were present in histology sections than initially suggested by HR-pQCT. The small size and heterogeneous presentation, limit the identification as VC on HR-pQCT.
\end{abstract}




\section{Introduction}

Erosions are the hallmark of bone destruction in rheumatoid arthritis (RA) ${ }^{1}$. They present as cortical interruptions on conventional radiography (CR), magnetic resonance imaging and ultrasound of the joints, and are the result of inflammatory tissue that stimulates osteoclastic bone resorption ${ }^{1,2}$. Another imaging technique is High-Resolution peripheral Quantitative Computed Tomography (HR-pQCT), which has a higher sensitivity than the gold standard $C R$ to detect cortical interruptions in finger joints ${ }^{3-5}$. Using HR-pQCT, cortical interruptions have been found in healthy subjects, some of which are hypothesized to be vascular channels $(\mathrm{VCs})^{1,6}$. Under normal conditions, the only documented peri-articular cortical interruptions in human anatomic specimens are blood vessels that perforate bone at the epiphysis ${ }^{7}$ and at the enthesis ${ }^{8}$. Vascular connections between bone marrow and the joint are an anatomical location for bone damage in animal models of arthritis ${ }^{8,9}$ as they provide direct entry points for synovitis and osteoclast-mediated joint destruction 9,10. In addition, angiogenesis, the de novo capillary outgrowth from pre-existing blood vessels, is a characteristic finding in RA and one of the initial features of the inflammatory response that facilitates migration of inflammatory cells ${ }^{11}$. It has therefore been hypothesized that bone damage in RA might start in VCs $1,9,10$. However, the actual presence of VCs in the finger joints has not been investigated in human subjects. We have previously shown that a heterogeneous pattern of cortical interruptions in finger joints can be seen by HR-pQCT6. The Study grouP for xtrEme Computed Tomography in Rheumatoid Arthritis (SPECTRA) collaboration has formulated a preliminary definition for VCs when using HR-pQCT imaging ${ }^{12}$. Using HR-pQCT, Boutroy et al. studied the SPECTRA definition for VCs in a barium perfusion study of an MCP joint and reported that two VCs detected on HRpQCT, were indeed a VC'13. However, it is unknown whether this proposed definition identifies VCs correctly and captures all VCs in an MCP joint.

The aim of this study was twofold: First, to study in MCP joints whether the cortical interruptions classified as VCs on HR-pQCT using the SPECTRA definition, are indeed VCs on histology sections. Second, to evaluate the image characteristics of histologically identified VCs on matched single slices of HR-pQCT images and on matched multiplane HR-pQCT images.

\section{Methods}

\section{Specimens}

Anatomic specimens of ten human fingers, each right hand index fingers of ten different individuals, were obtained from the Department of Anatomy and Embryology of the University of Amsterdam, Amsterdam, the Netherlands. The donors had dedicated their body by testament signed during life to the Department of Anatomy and Embryology of the University of Amsterdam, the Netherlands. A handwritten and signed codicil from each donor, posed when still alive and well, is kept at the Department of Anatomy and Embryology, University of Amsterdam, Amsterdam, the Netherlands. The medical history of the donors was unknown. 


\section{HR-pQCT image acquisition}

HR-pQCT (XtremeCT1, Scanco Medical AG, Switzerland) scans were performed at clinical in vivo settings, i.e. at $60 \mathrm{kVp}$ tube voltage, $900 \mu \mathrm{A}$ tube current, $100 \mathrm{~ms}$ integration time, and with a voxel size of $82 \mu^{6}$. The total region scanned was $144 \mathrm{~mm}$ (1760 slices) per finger from top distal phalangeal joint to proximal to the metacarpal head (Supplementary Figure 4.1). The total volumetric bone mineral density (vBMD) of the MCP joint (length in axial direction $18.04 \mathrm{~mm}$ ) was calculated using standard evaluation protocol of the manufacturer ${ }^{14}$. Scans of HR-pQCT were exported in Digital Imaging and Communications in Medicine (DICOM) format and analyzed using the Osirix (v.5.8.5 64-bit) multiplanar DICOM viewer, viewed on an iMAC 27 inch computer with $2560 \times 1440$ pixels.

\section{Selection of regions in MCP joints based on HR-pQCT images}

Prior to histologic examination, two readers independently scored the HR-pQCT images $^{6}$. One reader scored the images twice. The ten MCP joints were scored for the presence of cortical interruptions in at least two slices in two orthogonal planes (further referred as $2 \times 2$ slices). These cortical interruptions were, according to the SPECTRA collaboration, suspected to be a VC when a cortical interruption was characterized by a parallel cortical lining, present on at least $2 \times 2$ slices (Supplementary Figure 4.2)13,15. Based on the presence (kappa, $\mathrm{k}$ ) and the total number of cortical interruptions (intraclass correlation coefficient, ICC), intra-reader reliability was moderate to substantial (range $0.52-0.75$ ). Inter-reader reliability for the presence and total number of cortical interruptions was fair to moderate (range $0.37-0.55)^{6}$. Subsequently, four peri-articular regions in three MCP joints (average $3.4 \mathrm{~mm}$ ) with the highest number of cortical interruptions were selected for histological sectioning (Figure 4.1).

\section{Histology preparation and histology image analysis}

The four selected regions in MCP joints were fixed in formalin followed by dehydration in alcohol, propanol and finally xylene. Subsequently the joint was infiltrated with methylmethacrylate and embedded undecalcified in methylmethacrylate for sectioning. Fourteen- $\mu m$-thick sections were cut, transversal to the length of the finger, on a microtome (Reichert Jung GmbH, Heidelberg, Germany). Every second section was stained with Masson-Goldner trichrome, resulting in 478 histology sections in total. Subsequently all the sections were analyzed with a microscope (Nikon ECLIPSE 80i, Tokyo, Japan) equipped with a motorized Proscan 11 stage (Prior, Cambridge, UK), a MT1201 microcator (Heidenhain, Traunreit, Germany) and a DP72 digital camera (Olympus, Tokyo, Japan). The microscope system was connected to a PC with the stereological software newCAST which was used to visualize the sections on the PC screen (version 4.2.1.0, Visiopharm, Hørsholm, Denmark). A total magnification of 23 was used to visualize cortical interruptions and subsequently a magnification of 230 to visualize VCs. First, cortical interruptions that fulfilled the SPECTRA definition for a VC 
on HR-pQCT were examined for confirmation on histology. Second, and independently of HR-pQCT results, a histologist systematically analyzed the 478 histology sections for the presence of cortical interruptions. Subsequently, a cortical interruption was defined as a VC when it contained an artery and/or a vein. The maximum width of a histologically identified VC was measured using standard histology measurement methods.

\begin{tabular}{|c|c|c|c|c|c|c|}
\hline \multirow[t]{2}{*}{ A HR-pQCT coronal } & \multicolumn{6}{|c|}{$\begin{array}{l}\text { B Number of VCs (veins, and veins } \\
\text { accompanied by an artery) on histology in } \\
\text { sectioned regions and per joint side }\end{array}$} \\
\hline & & \multicolumn{4}{|c|}{ palmar ulnar dorsal radial } & total \\
\hline & \multirow{2}{*}{$\begin{array}{l}\text { veins } \\
\text { veins with } \\
\text { an artery }\end{array}$} & \multirow{2}{*}{$\begin{array}{l}5 \\
2\end{array}$} & \multirow{2}{*}{$\begin{array}{l}10 \\
1\end{array}$} & \multirow{2}{*}{$\begin{array}{l}4 \\
2\end{array}$} & \multirow{2}{*}{$\begin{array}{l}3 \\
2\end{array}$} & 22 \\
\hline & & & & & & 7 \\
\hline \multirow{4}{*}{ PB } & \multirow{2}{*}{$\begin{array}{l}\text { veins } \\
\text { veins with } \\
\text { an artery }\end{array}$} & 7 & 0 & 2 & 0 & 9 \\
\hline & & 2 & 0 & 0 & 0 & 2 \\
\hline & \multirow{2}{*}{$\begin{array}{l}\text { veins } \\
\text { veins with } \\
\text { an artery }\end{array}$} & 0 & 2 & 0 & 0 & 2 \\
\hline & & 0 & 1 & 0 & 0 & 1 \\
\hline
\end{tabular}

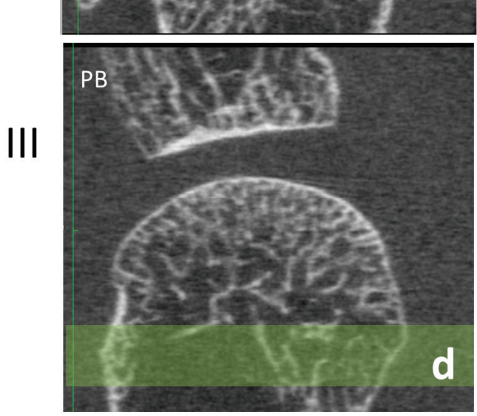

\begin{tabular}{llllll}
\hline $\begin{array}{l}\text { veins } \\
\text { veins with } \\
\text { an artery }\end{array}$ & 0 & 0 & 0 & 1 & 1 \\
\hline $\begin{array}{l}\text { ber of veins } \\
\text { veins with }\end{array}$ & 18 & 18 & 10 & 6 & 52 \\
& 4 & 2 & 2 & 3 & 11
\end{tabular}

Figure 4.1. Evaluated regions and distribution of vascular channels.

Panel I and II show four regions sectioned ( $a, b$, and $c$ ) in the phalangeal base. Region $c$ contained subchondral bone. Panel III shows a region sectioned (d) proximal to the MCP joint. The table (B) shows the distribution of vascular channels (veins, including veins accompanied by an artery) on HR-pQCT and the distribution on palmar, ulnar, dorsal and radial sides. Abbreviations: MCP; metacarpophalangeal, HR-pQCT; High Resolution peripheral Quantitative Computed Tomography, PB; phalangeal base, VCs; vascular channels 
Image analysis of histologically identified VCs on matched single HR-pQCT slices The transverse histology sections, in which a VC was identified, were visually matched and compared to corresponding single transverse HR-pQCT slices. Matching was based on the location and similarities of the surrounding normal bone structures and on the distance within the selected regions. All images were analyzed by one reader for the identification of a full cortical interruption or a periosteal excavation without full interruption of the cortex.

Image analysis of histologically identified VCs on matched multiplane HR-pQCT images In addition to the matched single slice examination, histologically identified VCs were also compared to matched multiplane HR-pQCT images by two readers in order to specify the image characteristics of histologically identified VCs in terms of the presence of a cortical interruption in at least two consecutive slices in two planes $(2 \times 2)$ or less (1x2) and whether they fulfilled the SPECTRA definition of a VC'13,15.

\section{Statistical analysis}

Descriptive statistics and frequencies were used to calculate the mean with SD for continuous data and the number of VCs. Independent samples t-test was used to compare width measured on histology between VCs containing veins only or both veins and arteries. Analyses were performed with SPSS Statistics for Windows version 23.0 (IBM Corp., Armonk, NY).

\section{Results}

The mean age of the specimen's subjects was 84.7 (SD 5.5) years. Regions selected for histology are shown in Figure 4.1. The average total $\mathrm{VBMD}$ of the three MCP joints was $219.7 \mathrm{mgHA} / \mathrm{cm}^{3}$.

In the selected regions of the MCP joints, seven cortical interruptions suggestive for a VC according to the SPECTRA criteria were detected on HR-pQCT. When comparing these images to histology, two were not evaluable on histology (due to poor quality of the histology section), in four cases no cortical interruptions could be found on histology and only one was found to be a VC on histology (Supplementary Figure 4.3).

In contrast, in the 478 14-um-thick sections that were examined histologically, 52 VCs were identified, and five cortical interruptions without any veins or arteries. All $52 \mathrm{VCs}$ contained a vein, of which 11 also an artery. The veins were located at the unar $(n=18)$, radial $(n=6)$, dorsal $(n=10)$ and palmar sides $(n=18)$ (Figure 4.1). Accompanying arteries were located at the palmar $(n=4)$, ulnar $(n=2)$, dorsal $(n=2)$ and radial $(n=3)$ sides. The width of VCs on histology ranged from 0.049 to $0.790 \mathrm{~mm}$ (mean $0.273 \mathrm{~mm}, \mathrm{SD} 0.177 \mathrm{~mm}$ ). The mean width of histologically identified VCs was significantly larger in VCs containing both veins and arteries (mean width $0.408 \mathrm{~mm}$, range $0.146-0.790 \mathrm{~mm}$ ) than those containing veins only (mean width $0.236 \mathrm{~mm}$, range: $0.049-0.670 \mathrm{~mm}, p<0.05)$. Six histologically identified VCs $(11.5 \%)$ were smaller than the HR-pQCT voxel size of $82 \mu \mathrm{m}$. 
All histology sections containing a VC could be visually matched to corresponding single transverse HR-pQCT slices. In $25(48.1 \%)$ of the histologically identified VCs, single matched HR-pQCT slices showed full cortical interruptions that penetrated the cortex in a straight (Figure 4.2 Panel I a) or oblique direction (Figure 4.2 Panel I b). In $14(26.9 \%)$ of the histologic identified VCs, single matched HR-pQCT slices showed a clear periosteal excavation without complete interruption of the cortex. These were identified as simple excavations, reflecting periosteal entrance of the vessel (Figure 4.2 Panel II a), or complex in configuration, following the intra-cortical curvature of the vessel (Figure 4.2 Panel II b). In $13(25 \%)$ of the histologically identified VCs, no clear cortical interruption or excavation could be identified on single matched HR-pQCT slices (Figure 4.2 Panel III a and b).

Histology

A

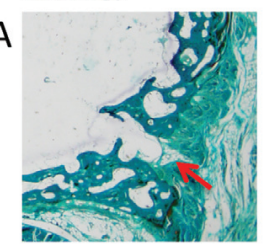

B

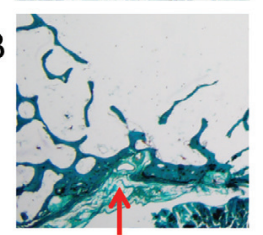

II

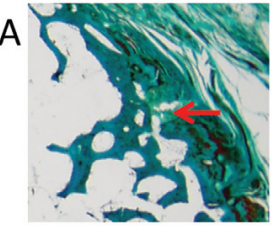

B

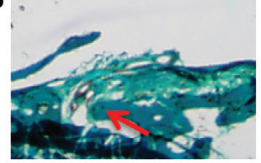

III A

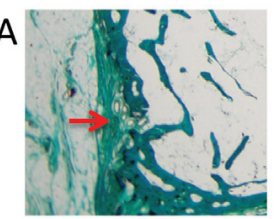

B

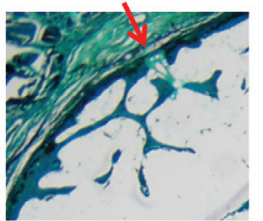

HR-pQCT
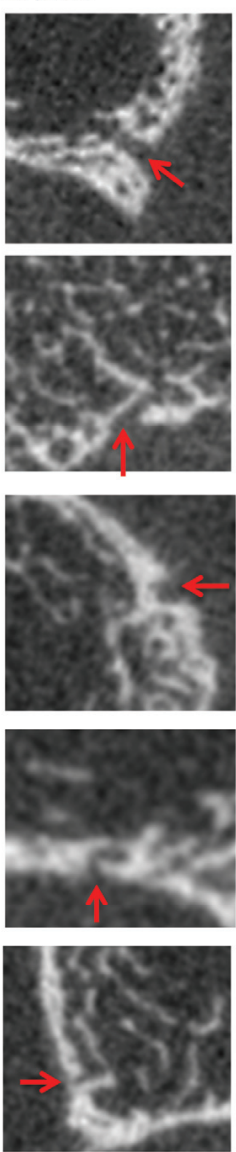

Figure 4.2. Histology sections and HR-pQCT slices of vascular channels.

Panel I. Histologically identified vascular channels with cortical interruptions on HR-pQCT slices with full cortical interruptions, that penetrated the cortex in a straight $(A)$ or oblique $(B)$ direction on HR-pQCT.

Panel II. Histologically identified vascular channels with a clear periosteal excavation without complete interruptions of the cortex on HR-pQCT, being simple excavations (A), or complex in configuration, following the intracortical curvature of the vessel (B).

Panel III. Histologically identified vascular channels with no clear cortical interruptions on HR-pQCT. 
The 52 histology sections with a VC were further compared to matched multiplane HRpQCT images. Eleven $(21 \%)$ of the histologically identified VCs showed cortical interruptions in at least $2 \times 2$ slices, $36(69 \%)$ in at least $1 \times 2$ slices and no cortical interruption in five (10\%). Five of the 11 cortical interruptions on $2 \times 2$ slices and 15 of the 36 cortical interruptions on $1 \times 2$ slices had a parallel cortical bone lining on HRpQCT. Figure 4.3 shows two examples of matched histology slices to HR-pQCT images.

Histology axial

a

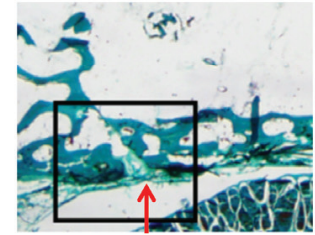

b

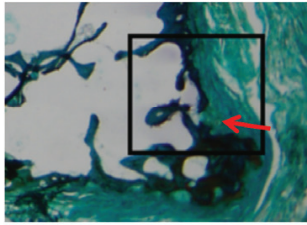

HR-pQCT multiplane axial
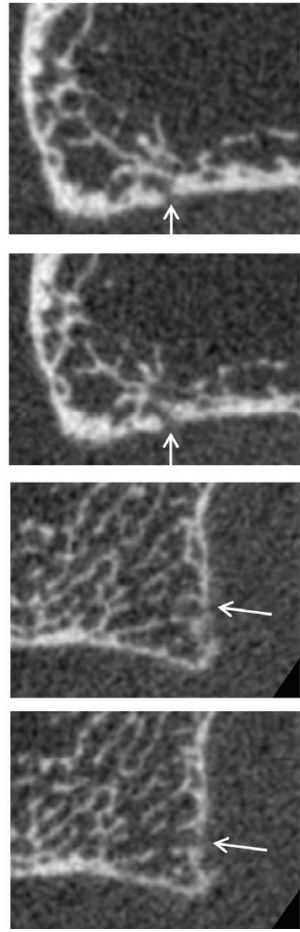

coronal
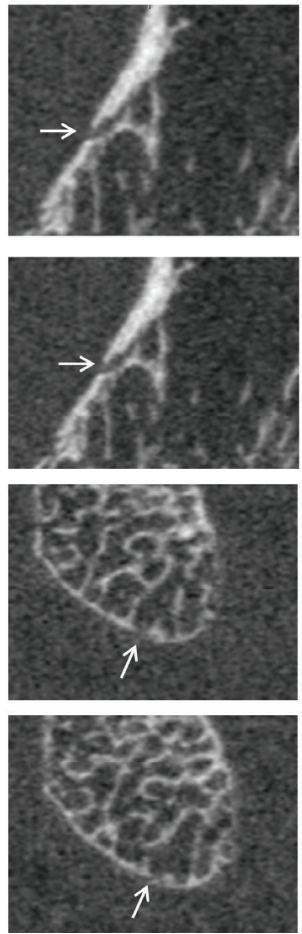

sagittal
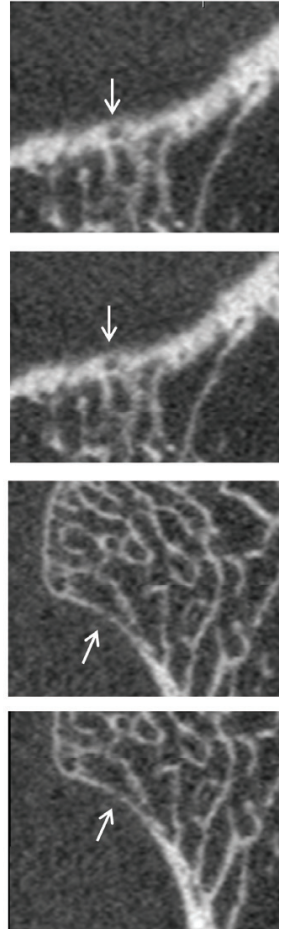

Figure 4.3. Examples of vascular channels identified on histology with matched multiplane HR-pQCT images of a vascular channel (panel a), and an interruption in the cortex not defined as a vascular channel according to SPECTRA (panel b).

\section{Discussion}

In the selected periarticular regions of MCP joints of anatomic specimens, substantially more VCs were identified on histology than initially expected from HR-pQCT images ${ }^{13}$. These histologically identified VCs were heterogeneous and many also small, making them difficult to identify on HR-pQCT. The finding of $52 \mathrm{VCs}$ on histology was unexpected, as the initial analyses of HR-pQCT images based on the SPECTRA criteria only suggested the presence of seven VCs and an earlier perfusion study only found two $\mathrm{VCs}^{13}$. This study is the first to formally indicate the ample presence of histologically identified VCs in MCP joints. Our findings have consequences for the interpretation of $H R-p Q C T$ images and can be of clinical significance. 
First, the VCs were heterogeneous in configuration on HR-pQCT. VCs presented as full cortical interruptions (straight or oblique penetration of the cortex) or excavations (simple or complex configuration). The heterogeneity of VC images on HR-pQCT is in line with earlier findings that reported variable image characteristics of cortical interruptions on HR-pQCT3,16. By using predefined image characteristics of VCs according to SPECTRA, seven cortical interruptions fulfilled the SPECTRA definition of a VC (i.e. parallel cortical lining on two consecutive slices in two planes) on HR-pQCT, five could be evaluated on histology and only one was a true VC on histology. Therefore, the current definition of a VC on HR-pQCT imaging appears insufficient to correctly identify VCs. The heterogeneity of the VCs on HR-pQCT needs to be incorporated in the development of a new definition.

Second, the histologically identified VCs were small in size with a mean width of 0.273 $\mathrm{mm}$. While in a barium perfusion experiment using HR-pQCT only two VCs were identified, such perfusion studies are presumably insufficient to detect small VCs ${ }^{13}$. In this study we used histologically identified VCs and matched them to their corresponding location on HR-pQCT images. In $13(25 \%)$ of the histologically identified $V C s$, no clear cortical interruption or excavation could be identified on single matched HR-pQCT slices and in $5(10 \%)$ of the histologically identified VCs, no cortical interruption was seen on multiplane HR-pQCT images. On histology, six identified VCs $(11.5 \%)$ were smaller than the HR-pQCT voxel size of $82 \mu \mathrm{m}$. The small size of the VCs and voxel size of HR-pQCT explains the observation that multiple VCs could not be identified as cortical interruptions on HR-pQCT. The smallest details that can be seen on the HR-pQCT scans are $82 \mu \mathrm{m}$ (the voxel size). Cortical interruptions smaller than this size are only partly covered by a voxel ('partial volumes') and may not be detectable. On the other hand, very thin cortices may be identified as an interruption on HR-pQCT scanning. To investigate the potential errors involved due to these partial volume effects, we compared in an earlier study the detection of cortical interruptions on HR-pQCT imaging with that on micro computed tomography images with a much higher resolution $(18 \mu \mathrm{m} \text { voxel size })^{6}$. In that study, we found that the sensitivity of HR-pQCT for detecting cortical interruptions was $81.6 \%$ positive predictive value was also $81.6 \%$ ) while the specificity was $64.0 \%$. No sensitivity can be calculated from the comparison with histology since the evaluations on HR-pQCT and histology were independently performed with different aims for each modality. For clinical application, the previously obtained sensitivity and specificity values were considered acceptable. When developing a new definition, for example including cortical interruptions on less than $2 \times 2$ slices, the small size and voxel size of HR-pQCT should be taken into consideration. Visual interpretation of HR-pQCT images is a complex task, as has been shown in studies that evaluated trabecular bone structure and the lining of the endocortical bone ${ }^{17}$. In the field of metabolic bone diseases, this has been solved by fully or semi-automated image analysis algorithms. We are currently developing computer-assisted semi-automatic and fully automatic algorithms that might facilitate evaluation of the presence, size and location of cortical interruptions and VCs. 
Third, the VCs were spread around the joint, which is the main location for inflammation in RA. It has previously been shown in animal models of arthritis and hypothesized in humans, that vascular connections can play a pathophysiologic role in the formation of erosions when joint inflammation occurs $9,10,18$. Two different scenarios have been proposed for the pathogenesis of peri-articular erosions in RA; the inside-out and outside-in scenarios ${ }^{19}$. In the conventional view, the outside-in concept hypothesizes that RA starts with inflammation in the synovial membrane, spreading locally to adjacent structures including the bone marrow through cortical interruptions ${ }^{19}$. The high number of histologically identified peri-articular VCs supports that inflammation can indeed spread locally through VCs to the bone marrow from these joint locations. The alternative view is the inside-out scenario, which hypothesizes that inflammation starts in the bone marrow and migrates locally through peri-articular cortical interruptions into the joint ${ }^{19}$. VCs facilitate local homing of osteoclast precursor cells, which upon contact with the appropriate molecular signals such as anti-citrullinated protein antibody (ACPA), can differentiate into osteoclasts in the preclinical phase of RA ${ }^{1}$. The high number of VCs around MCP joints in our study could explain why for example ACPA positive subjects develop peri-articular erosions before the occurrence of synovitis.

This study has several limitations. First, the study was conducted in a sample of anatomic specimens of elderly women with unknown medical background, preserved in formalin, and with low mineralized bone. The relatively old age might have affected the condition and visual observations on HR-pQCT imaging of the fingers. Average vBMD in the specimens was $219.7 \mathrm{mgHA} / \mathrm{cm}^{3}$, which is lower than observed in the normal population $\left(>300 \mathrm{mgHA} / \mathrm{cm}^{3}\right)^{5,20}$. Second, only MCP joints were studied and the results might therefore not be applicable to other joints. However, we deliberately selected MCP joints, as these joint locations are frequently the first to be involved in the development of RA and where erosions might occur even before RA becomes clinical ' The strength of this study is that imaging by histology and HR-pQCT was performed independently.

To conclude, substantially more VCs were present in histologic sections than initially suggested by HR-pQCT. Although most histologic VCs could be identified as cortical interruptions in matched HR-pQCT slices, their small size and heterogeneous presentation, limit the identification as VC on HR-pQCT images. New definitions for $V C$ s on HR-pQCT should take this into consideration. 


\section{References}

1 Schett, G. \& Gravallese, E. Bone erosion in rheumatoid arthritis: mechanisms, diagnosis and treatment. Nat Rev Rheumatol 8, 656-664 (2012).

2 Ostergaard, M., Pedersen, S. J. \& Dohn, U. M. Imaging in rheumatoid arthritis-status and recent advances for magnetic resonance imaging, ultrasonography, computed tomography and conventional radiography. Best Pract Res Clin Rheumatol 22, 1019-1044 (2008).

3 Stach, C. M. et al. Periarticular bone structure in rheumatoid arthritis patients and healthy individuals assessed by high-resolution computed tomography. Arthritis Rheum 62, 330-339 (2010). Srikhum, W. et al. Quantitative and semiquantitative bone erosion assessment on high-resolution peripheral quantitative computed tomography in rheumatoid arthritis. J Rheumatol 40, 408-4 16 (2013). Fouque-Aubert, A. et al. Assessment of hand bone loss in rheumatoid arthritis by high-resolution peripheral quantitative CT. Ann Rheum Dis 69, 1671-1676 (2010).

Scharmga, A. et al. Visual detection of cortical breaks in hand joints: reliability and validity of highresolution peripheral quantitative CT compared to microCT. BMC Musculoskelet Disord 17, 271 (2016).

Wideman, R. F. \& Prisby, R. D. Bone circulatory disturbances in the development of spontaneous bacterial chondronecrosis with osteomyelitis: a translational model for the pathogenesis of femoral head necrosis. Front Endocrinol (Lausanne) 3, 183 (2012).

Benjamin, M. et al. Microdamage and altered vascularity at the enthesis-bone interface provides an anatomic explanation for bone involvement in the HLA-B27-associated spondylarthritides and allied disorders. Arthritis Rheum 56, 224-233 (2007).

Binks, D. A. et al. Role of vascular channels as a novel mechanism for subchondral bone damage at cruciate ligament entheses in osteoarthritis and inflammatory arthritis. Ann Rheum Dis 74, 196-203 (2015). Schett, G. et al. Analysis of the kinetics of osteoclastogenesis in arthritic rats. Arthritis Rheum 52, $3192-$ 3201 (2005).

11 Tas, S. W., Maracle, C. X., Balogh, E. \& Szekanecz, Z. Targeting of proangiogenic signalling pathways in chronic inflammation. Nat Rev Rheumatol 12, 111 1-122 (2016).

12 Barnabe, C. et al. Definition for Rheumatoid Arthritis Erosions Imaged with High Resolution Peripheral Quantitative Computed Tomography and Interreader Reliability for Detection and Measurement. J Rheumatol 43, 1935-1940 (2016). Boutroy, S. et al. Erosion or vessel channel? Arthritis Rheumatol (2015).

14 Boutroy, S., Bouxsein, M. L., Munoz, F. \& Delmas, P. D. In vivo assessment of trabecular bone microarchitecture by high-resolution peripheral quantitative computed tomography. J Clin Endocrinol Metab 90, 6508-6515 (2005).

15 Finzel, S. et al. A detailed comparative study of high-resolution ultrasound and micro-computed tomography for detection of arthritic bone erosions. Arthritis Rheum 63, 1231-1236 (2011). and Healthy Controls Imaged by High-resolution Peripheral Quantitative Computed Tomography. J Rheumatol 43, 1914-1920 (2016).

Zebaze, R. \& Seeman, E. Cortical bone: a challenging geography. J Bone Miner Res 30, 24-29 (2015). Marinova-Mutafchieva, L., Williams, R. O., Funa, K., Maini, R. N. \& Zvaifler, N. J. Inflammation is preceded by tumor necrosis factor-dependent infiltration of mesenchymal cells in experimental arthritis. Arthritis Rheum 46, 507-513 (2002). Schett, G. \& Firestein, G. S. Mr Outside and Mr Inside: classic and alternative views on the pathogenesis of rheumatoid arthritis. Ann Rheum Dis 69, 787-789 (2010).

20 Feehan, L., Buie, H., Li, L. \& McKay, H. A customized protocol to assess bone quality in the metacarpal head, metacarpal shaft and distal radius: a high resolution peripheral quantitative computed tomography precision study. BMC Musculoskelet Disord 14, 367 (2013). 


\section{Supplementary files}

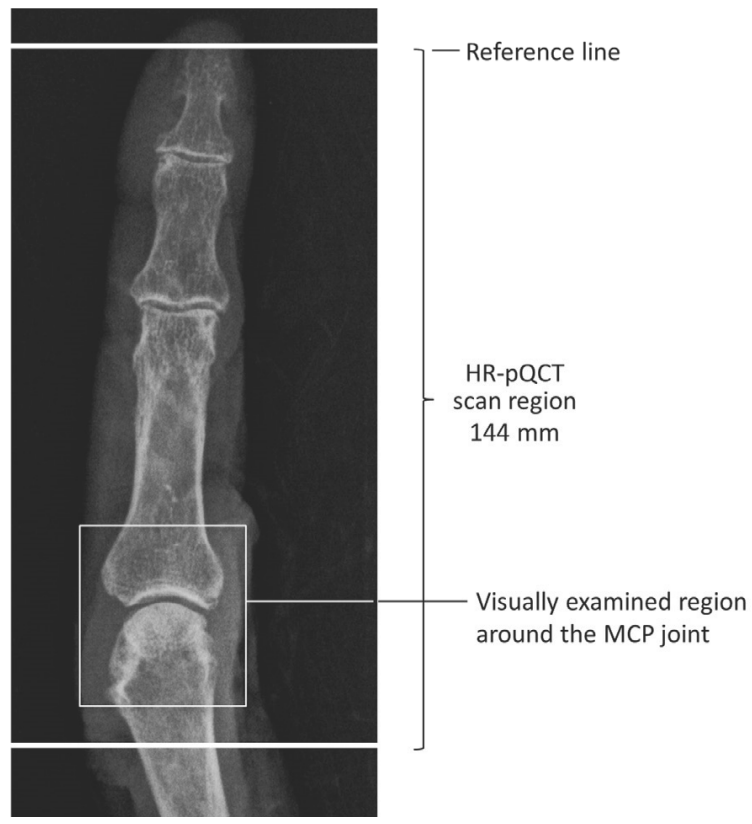

Supplementary Figure 4.1.

Scan region of index finger and visually examined region on HR-pQCT

Abbreviations: HR-pQCT; High Resolution peripheral Quantitative Computed Tomography

HR-pQCT transversal

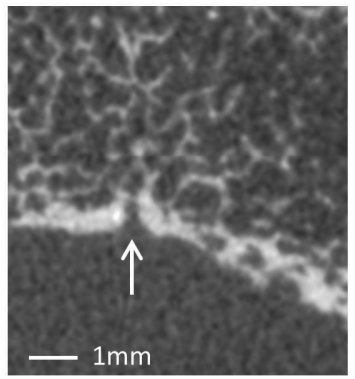

HR-pQCT coronal

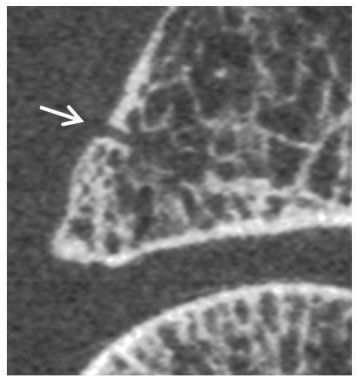

HR-pQCT sagittal

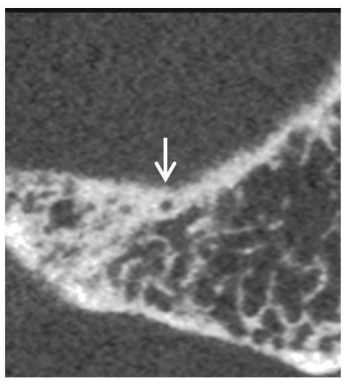

Supplementary Figure 4.2.

Example of a cortical interruption in the MCP joint in transversal, coronal and sagittal slices that was classified as a vascular channel according to the SPECTRA definition, based on having a parallel structure (arrow).

Abbreviations: MCP; metacarpophalangeal, HR-pQCT; High Resolution peripheral Quantitative Computed Tomography, SPECTRA; The Study grouP for xtrEme Computed Tomography in Rheumatoid Arthritis 
HR-pQCT axial

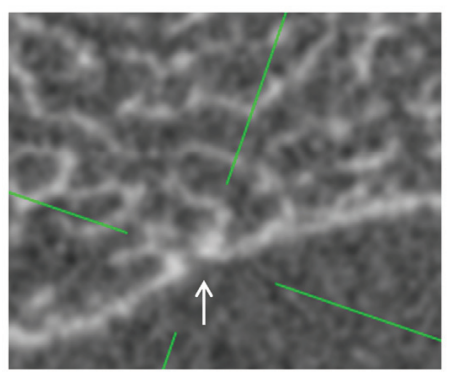

Histology axial

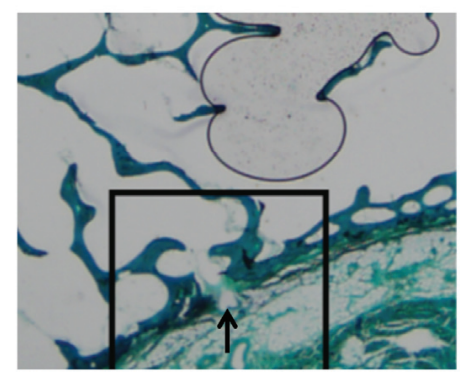

Supplementary Figure 4.3.

Example of a VC as defined on HR-pQCT with confirmation on histology.

An interruption defined VC (arrow) by HR-pQCT (left) and confirmed as VC (arrow) on histology (right) Abbreviations: HR-pQCT; High Resolution peripheral Quantitative Computed Tomography, VC; vascular channel 
Development of a scoring method to visually score cortical interruptions on high-resolution peripheral quantitative computed tomography in rheumatoid arthritis and healthy controls
A. Scharmga, M. Peters, J.P. van den Bergh,
P. Geusens, D. Loeffen, B. van Rietbergen,
T. Schoonbrood, D. Vosse, R. Weijers,
A. van Tubergen

Submitted 


\begin{abstract}
Objectives. To develop a scoring method to visually score cortical interruptions in finger joints on High-Resolution peripheral Quantitative Computed Tomography (HR$p Q C T)$, determine its intra- and inter-reader reliability and test its feasibility.

Methods. The scoring method was developed by integrating results from in-depth discussions with experts, consensus meetings, multiple reading experiments and the literature. Cortical interruptions were scored by two independent readers in an imaging dataset with finger joints from patients with rheumatoid arthritis (RA) and healthy controls and assessed for adjacent trabecular distortion. Reliability for the total number of cortical interruptions per joint and per quadrant was calculated using intraclass correlation coefficient (ICC). Feasibility was tested by recording the time to analyze one joint.

Results. In 98 joints we identified 252 cortical interruptions, 17\% had trabecular distortion. Mean diameter of the interruptions was significantly larger in patients with RA compared with healthy controls $(0.88$ vs $0.47 \mathrm{~mm}, \mathrm{p}=0.03)$. Intra-reader reliability was ICC 0.88 (95\% Cl 0.83;0.92) per joint and ICC 0.69 (95\% Cl 0.65;0.73) per quadrant. Inter-reader reliability was ICC $0.48(95 \% \mathrm{Cl} 0.20 ; 0.67)$ per joint and ICC $0.56(95 \% \mathrm{Cl} 0.49 ; 0.62)$ per quadrant. The time to score one joint was mean 9.2 (SD 4.9) $\mathrm{min}$.
\end{abstract}

Conclusions. This scoring method allows detection of small cortical interruptions on HRpQCT imaging of finger joints, which is promising for use in clinical studies. 


\section{Introduction}

High-Resolution peripheral Quantitative Computed Tomography (HR-pQCT) is a noninvasive imaging technique enabling three dimensional analysis of bone microarchitecture at an isotropic voxel size of 82 microns and a spatial resolution of 130 microns. HR-pQCT has the potential to identify and quantify early bone changes in metacarpophalangeal (MCP) joints before bone damage can be identified on radiographs ${ }^{1}$. With HR-pQCT, very small cortical interruptions of less than $0.5 \mathrm{~mm}$ can be detected ${ }^{1,2}$. These cortical interruptions can be physiological, e.g. vascular channels or pathological, e.g. erosions in rheumatic diseases ${ }^{3}$. A definition for detection of a vascular channel on HR-pQCT has been proposed ${ }^{3}$, but appeared insufficient ${ }^{4}$. Vascular channels were more heterogeneous and smaller in size than previously suggested. Also several definitions for erosion on HR-pQCT exist ${ }^{3}$. In one study cortical interruptions greater than $1.9 \mathrm{~mm}$ were considered bone erosions specific for rheumatoid arthritis (RA)'. To date, a validated visual scoring method for HR-pQCT images that incorporates all cortical interruptions, including vascular channels and erosions, is lacking. Such a scoring system could aid in making a distinction between (small) physiological vascular channels and pathological erosions, which may be accompanied by adjacent trabecular distortion ${ }^{3}$.

Here we describe the development of a scoring method to visually score cortical interruptions on HR-pQCT. We integrated results from in-depth discussions with experts, consensus meetings, multiple reading experiments 4,5 , and the literature on an image grading scale for motion artefacts ${ }^{6}$ and joint assessment on HR-pQCT images as described by Stach et al $^{1}$. Furthermore, we tested its intra- and inter-reader reliability, and feasibility in terms of time to perform.

\section{Materials and methods}

\section{Subjects}

A representative sample of 30 subjects (10 healthy controls and 20 patients with RA) from 38 female healthy controls and 41 female patients diagnosed with RA, participating in an observational cohort study, the MOSA-Hand study, was used'. All patients with RA fulfilled the 2010 American College of Rheumatology (ACR)/European League Against Rheumatism (EULAR) classification criteria for RA ${ }^{8}$. Healthy controls, matched per decade, did not suffer from hand joint complaints. All subjects were recruited at the Maastricht University Medical Centre, the Netherlands and signed informed consent. Ethical approval was obtained from the ethics board of the academic hospital Maastricht/ Maastricht University, the Netherlands. Netherlands Trial Registry number: NTR3821

\section{Conventional radiography}

Posterior-anterior radiographs of both hands were taken from all subjects at baseline. Two experienced rheumatologists (TS and DV) independently scored the radiographs according to the Sharp/ van der Heijde method (SvdH) for the presence of joint 
damage in hand joints (range 0-280) 9 . Radiographs were scored using a free Digital Imaging and Communications in Medicine (DICOM) viewer (Osirix v.5.8.5 64-bit). The readers were blinded for demographic and clinical data. The 30 subjects used in this study were selected based on the severity of radiographic damage according to the $\mathrm{SvdH}$ score. Healthy controls were selected when the SvdH score was zero. In patients with RA, the selection was based on representing a wide spectrum of damage according to the $\mathrm{SvdH}$ scores, ranging from none to severe.

\section{HR-pQCT image acquisition}

Second and third MCP and proximal interphalangeal (PIP) joints were scanned with HRpQCT (XtremeCT1, Scanco Medical AG, Switzerland) according to the HR-pQCT imaging protocol proposed by The Study grouP for xtrEme Computed Tomography in Rheumatoid Arthritis (SPECTRA) at baseline and after one year ${ }^{10}$. In patients with RA, both hands were scanned and in healthy controls only the dominant hand. Each hand was scanned using the standard carbon forearm cast as provided by the manufacturer. Scanning time per patient was nine minutes for MCP joints (three stacks of $9.02 \mathrm{~mm}$, 330 slices) and six minutes for PIP joints (two stacks of 9.02 mm, 220 slices).

\section{Development of the scoring method}

We developed a scoring method to visually score cortical interruptions on HR-pQCT images. We integrated results from in-depth discussions with experts, consensus meetings, multiple reading experiments and comparison with microCT and histology 4,5 and from the literature available studies on the grading of motion artefacts 6 and on the assessment of finger joints using HR-pQCT images ${ }^{1}$. The experts consisted of a panel of rheumatologists, radiologists and engineers specialized in image analyses.

Our proposal for the visual scoring method is shown in online supplementary Figure 5.1.

First, images need to be assessed for the presence of motion artifacts according to Pialat et al. ${ }^{\circ}$. Only HR-pQCT images with grades 1, 2 or 3 are considered evaluable, and image grades 4 and 5 are excluded from further analysis. Second, each joint is divided into its proximal and distal bone end and it is assessed whether a normal outer bone contour is present. When more than $50 \%$ of the joint surface of the cortical bone per bone end is destroyed, the bone end is considered 'totally destructed' and excluded from further analysis (Figure 5.1).
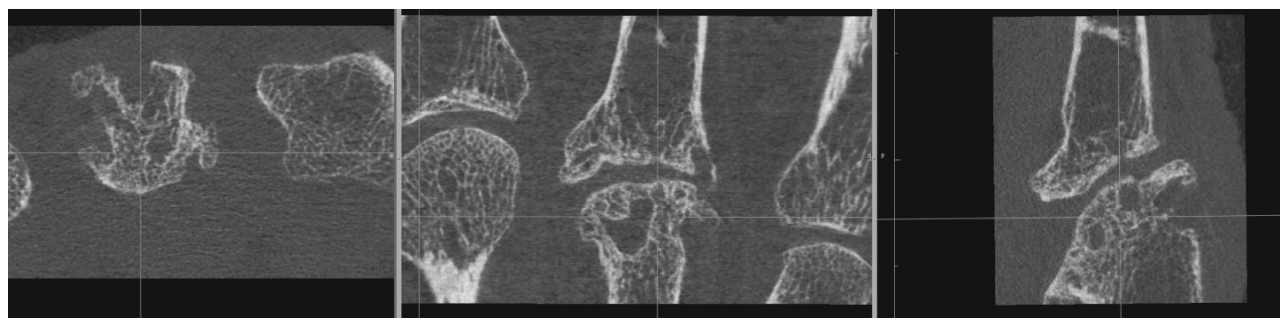

Figure 5.1 
Third, each bone end is further divided into four quadrants (palmar, ulnar, dorsal and radial) according to Stach et al ${ }^{1}$ (Figure 5.2).

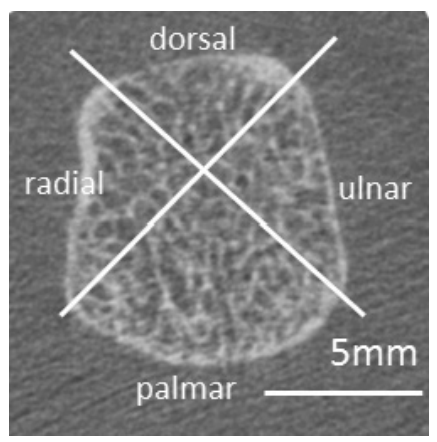

Figure 5.2

Thus per joint, eight quadrants are assessed for the presence of cortical interruptions'. Fourth, a cortical interruption is scored when it fulfilled one of the following criteria: a). a cortical interruption in at least one slice in one plane and in addition in two consecutive slices in another plane (i.e. $\geq 1 \times \geq 2$ or $\geq 2 x \geq 1$ slices), b). a cortical interruption in more than one consecutive slice in one plane and in more than one slice in another plane (e.g. $\geq 2 x \geq 2$ slices). Fifth, when a cortical interruption is identified, its maximal diameter is measured (in $\mathrm{mm}$ ) in one of the three planes (transverse, coronal or sagittal). Sixth, the adjacent trabecular structure is assessed for distortion, defined as clear absence of calcified trabeculae underlying the cortical interruption (Figure 5.3).
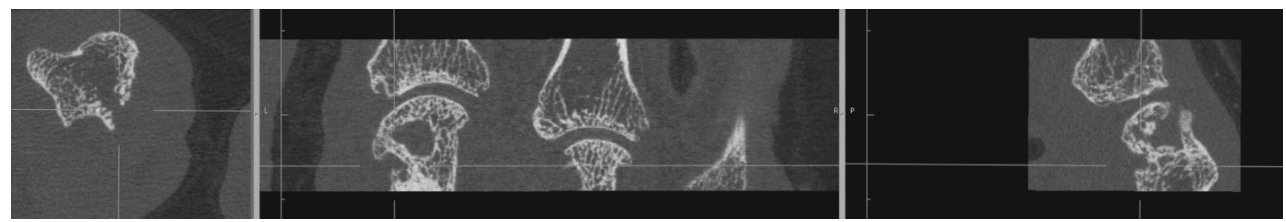

Figure 5.3

\section{Image analyses}

The HR-pQCT images were viewed in Osirix. Using the visual scoring method, two experienced readers (AS and MP) scored the HR-pQCT images twice, blinded for demographic and clinical data, and independently of each other. When large discrepancies (difference of $>3$ cortical interruptions per joint) between the readers were found, these images were checked and evaluated for reasons for discrepancy.

\section{Reliability}

For testing the reliability of the visual scoring method, second and third MCP and PIP joints were used. In the dataset, each healthy control contributed with two joints, one MCP and one PIP joint. Each patient with RA contributed with four joints, two MCP and two PIP joints which were randomly selected from either hand. 
Feasibility

For testing the feasibility of the visual scoring method, start and end time of reading one joint was recorded in each subject in full round minutes, for each reader separately.

\section{Statistics}

Descriptive statistics were used to calculate the $\mathrm{SvdH}$ score on conventional radiographs $(C R)$, and to analyze the number, diameter of cortical interruptions and presence of trabecular distortion, and the average time to score a joint on HR-pQCT using the visual scoring method. Wilcoxon signed-rank test was used to compare the number of cortical interruptions of the first and second reading on HR-pQCT for all subjects and for intrareader scores. Mann-Whitney $U$ test was used to compare the diameter of the cortical interruptions between healthy controls and patients with RA. Intra- and inter-reader reliability of the visual scoring method was assessed using Cohen's kappa ( $\mathrm{k}$ ) based on the presence or absence of a cortical interruption per quadrant and per joint. Intraclass correlation coefficient (ICC), based on a two way random effects model, was used to assess intra- and inter-reader reliability based on the total number of cortical interruptions per quadrant and per joint. Reliability was rated according to Landis et al.: <0.00 poor, 0.00-0.20 slight, 0.21-0.40 fair, 0.41-0.60 moderate, 0.61-0.80 substantial, 0.81-1.00 almost perfect'1 ${ }^{1}$. Totally destructed bone ends (base and/or head) were excluded from the analyses on HR-pQCT. Only quadrants without motion artefacts on both readings were taken into account for the intra-reader analysis.

\section{Results}

Online supplementary Figure 5.2 shows a flowchart of subject inclusion in the MOSAHand study and selection of the sample of 30 subjects $(10$ healthy controls and 20 patients with RA) for this study. The mean age was 46.1 (SD 9.2) years for the selected healthy controls and 57.4 (SD 5.8) years for the selected patients with RA. The mean disease duration for patients with RA was 117.1 (SD 1 10.1) months. In one patient, the HR-pQCT images of two PIP joints were missing because of intolerance to long immobilization during scanning. The dataset of HR-pQCT images to examine the reliability and feasibility of the visual method included in total 98 joints: 50 MCP (10 from healthy controls and 40 from patients with RA) and 48 PIP joints (10 from healthy controls and 38 from patients with RA).

\section{Conventional radiography}

Mean SvdH scores for the selected subjects were 0 (SD 0) for healthy controls and 15.1 (SD 22.6; range 0 - 87.5) for patients with RA.

\section{Image analysis}

Table 5.1 shows descriptives of the first and second reading of the dataset on HRpQCT. In the first readings of Reader 1 and Reader 2, 40 out of 784 quadrants were not considered evaluable due to motion grade $>3,32$ quadrants were considered to be 
totally destructed by Reader 1 and 42 by Reader 2. In total, 702 quadrants by Reader 1 and 706 quadrants by Reader 2 were further assessed for the presence of a cortical interruption fulfilling the criteria of the scoring method. Reader 1 identified 252 cortical interruptions, of which 35 were small (in at least $1 \times 2$ slices) and 217 were identified in $2 \times 2$ or more consecutive slices. Reader 2 scored a significantly higher number of cortical interruptions than Reader 1 in the first and second reading (first reading: 381 versus 252 cortical interruptions, second reading: 456 versus 248 cortical interruptions, both $\mathrm{p}<0.001)$. Intra-reader scores for the total number of cortical interruptions were not significantly different for Reader 1 (252 versus 248, $\mathrm{p}=0.67$ ), but were significantly different for Reader 2 (381 versus 456, $\mathrm{p}<0.001$ ). Adjacent trabecular distortion was observed in $17 \%$ (Reader 1) and $25 \%$ (Reader 2) of the cortical interruptions (Table 5.1). The diameter of the cortical interruptions ranged from $0.09 \mathrm{~mm}$ to $7.4 \mathrm{~mm}$ for Reader 1 and from $0.11 \mathrm{~mm}$ to $7.88 \mathrm{~mm}$ for Reader 2 (Table 5.1).

Table 5.1. Descriptives of first and second reading of Reader 1 and 2

\begin{tabular}{lllll}
\hline & & Reader 1 & & Reader 2 \\
& First & Second & First & Second \\
& reading & reading & reading & reading \\
\hline Number of evaluable quadrants (\%) & $734(94 \%)$ & $744(95 \%)$ & $728(93 \%)$ & $730(93 \%)$ \\
Number of totally destructed quadrants & 32 & 36 & 32 & 32 \\
Number of quadrants further assessed & $702(90 \%)$ & $708(90 \%)$ & $696(89 \%)$ & $698(89 \%)$ \\
Number of joints further assessed & 93 & 93 & 93 & 95 \\
Number of interruptions & 252 & 248 & 381 & 456 \\
$\quad \begin{array}{l}\text { Mean per joint (SD) } \\
\text { present on < 2 consecutive slices }\end{array}$ & $2.7(2.3)$ & $2.6(2.2)$ & $4.1(3.3)$ & $4.8(3.2)$ \\
present on $\geq 2$ consecutive slices & 35 & 45 & 1 & 0 \\
$\quad$ with trabecular distortion (\%) & 217 & 203 & 380 & 456 \\
Mean diameter of cortical interruption in mm (SD), & $0.82(0.91)$ & $0.73(0.89)$ & $0.68(0.71)$ & 0.63 (0.62) \\
range & $0.12-7.16$ & $0.09-7.4$ & $0.13-7.88$ & $0.11-4.57$ \\
Mean reading time per joint in minutes, (SD), range & $8.3(3.7)$ & $8.8(3.9)$ & $9.8(6.9)$ & $9.9(5.4)$
\end{tabular}

Table 5.2 shows the results of the visual scoring for healthy controls and patients with RA separately, based on results of the first reading of Reader 1. Most interruptions were seen in two or more than two consecutive slices in two or more planes ( $\geq 2 x \geq 2$ planes) in both healthy controls and patients with RA $(n=34(89 \%)$ and $n=183(85.6 \%)$, respectively). None of the cortical interruptions in healthy controls were accompanied by adjacent trabecular distortion, but in patients with RA $44(21 \%)$ of the cortical interruptions were accompanied by adjacent trabecular distortion. The mean diameter of the interruptions was significantly larger in patients with RA compared with healthy controls (0.88 vs $0.47 \mathrm{~mm}, \mathrm{p}=0.03$ ). 
Table 5.2. Descriptives of cortical interruptions in healthy controls and patients with RA

\begin{tabular}{lll}
\hline & $\begin{array}{l}\text { Healthy controls } \\
\text { (n=20 joints) }\end{array}$ & $\begin{array}{l}\text { Patients with RA } \\
\text { (n=78 joints) }\end{array}$ \\
\hline $\begin{array}{l}\text { Number of interruptions } \\
\text { Mean number of cortical interruptions per }\end{array}$ & $1.9(1.7)$ & 214 \\
joint (SD) & & $2.9(2.4)$ \\
present on < 2 consecutive slices(\%) & $4(11 \%)$ & $31(14.4 \%)$ \\
present on $\geq 2$ consecutive slices (\%) & $34(89 \%)$ & $183(85.6 \%)$ \\
with trabecular distortion (\%) & $0(0 \%)$ & $44(21 \%)$ \\
Mean diameter of cortical interruption in & $0.47(0.22)$ & $0.88(0.97)$ \\
mm, (SD), range & $0.19-1.11$ & $0.12-7.16$ \\
Mean reading time per joint in minutes, & $7.4(3.2)$ & $8.5(3.7)$ \\
(SD), range & $3-14$ & $1-18$
\end{tabular}

Numbers based on results Reader 1, first reading

Abbreviations: RA; rheumatoid arthritis

Online supplementary Table 5.1 shows the mean number of cortical interruptions per quadrant in healthy controls and patients with RA, based on results of the first reading of Reader 1. Significantly more cortical interruptions were observed in the ulnar and radial quadrants in patients with RA compared with healthy controls $(p<0.05$, Supplementary Table 5.1). Cortical interruptions were also frequently seen in the palmar quadrants, but equally in patients with RA and healthy controls. Cortical interruptions with adjacent trabecular distortion in patients with RA were most frequently seen in the ulnar quadrant $(n=17)$, followed by palmar, radial and dorsal quadrants ( $n=9, n=8$ and $n=5$ respectively).

In total 13 joints were re-evaluated because of discrepancies of $>3$ interruptions per joint between the two readers in the first reading. Discrepancies occurred only in patients with RA. In general, Reader 1 was more conservative than Reader 2 on the identification of an interruption. Online supplementary Figure 5.3, panel A shows an example of a discrepancy in the observation of a possible cortical interruption. The interruption is difficult to distinguish from the background noise, due to low density caused by low mineralization and/or a thin cortex. Furthermore, larger interruptions showed more agreement than smaller interruptions. Also, irregularities in the cortex or bone apposition (online supplementary Figure 5.3, panel B) caused discrepancies in the total number of interruptions. Exceptionally, an interruption was overlooked by one reader, multiple smaller interruptions were counted instead of one large interruption (online supplementary Figure 5.3, panel C) or, in retrospect, an interruption was wrongly considered a cortical interruption, but did not fulfill the criteria. 
Reliability

Table 5.3 shows the results for intra- and inter-reader reliability of the visual scoring method for the number of cortical interruptions based on joints $(n=98)$ and quadrants $(n=784)$. Intra-reader reliability of Reader 1 and Reader 2 was moderate 10.52 to $0.67)$ for the presence of an interruption, but substantial to almost perfect 10.69 to 0.88 ) for the number of cortical interruptions (Table 5.3). Inter-reader reliability was fair to moderate $(0.37$ to 0.56$)$ for the presence and total number of cortical interruptions (Table 5.3).

Feasibility

The mean time to score one joint was $8.6 \mathrm{~min}$ (SD 3.7) for Reader 1 and $10.0 \mathrm{~min}$ (SD 6.0) for Reader 2 (Table 5.1), the mean for both readers was $9.2 \mathrm{~min}$ (SD 4.9).

Table 5.3. Intra- and inter-reader reliability of the visual scoring method

\begin{tabular}{|c|c|c|c|c|c|c|}
\hline & \multicolumn{4}{|c|}{ Intra-reader } & \multicolumn{2}{|c|}{ Inter-reader (reading 1) } \\
\hline & Rea & 1 & $\operatorname{Re}$ & $\begin{array}{l}2 \\
\text { ICC }\end{array}$ & K & ICC \\
\hline $\begin{array}{l}\text { Cortical } \\
\text { interruptions } \\
\text { based on joints }\end{array}$ & $\begin{array}{l}0.52 \\
(0.26 ; 0.79)\end{array}$ & $\begin{array}{l}0.88 \\
(0.83 ; 0.92)\end{array}$ & $\begin{array}{l}0.56 \\
(0.07 ; 1.00)\end{array}$ & $\begin{array}{l}0.79 \\
(0.66 ; 0.87)\end{array}$ & $\begin{array}{l}0.37 \\
(0.02 ; 0.72)\end{array}$ & $\begin{array}{l}0.48 \\
(0.20 ; 0.67)\end{array}$ \\
\hline $\begin{array}{l}\text { Cortical } \\
\text { interruptions } \\
\text { based on } \\
\text { quadrants }\end{array}$ & $\begin{array}{l}0.63 \\
(0.56 ; 0.69)\end{array}$ & $\begin{array}{l}0.69 \\
(0.65 ; 0.73)\end{array}$ & $\begin{array}{l}0.67 \\
(0.62 ; 0.72)\end{array}$ & $\begin{array}{l}0.76 \\
(0.72 ; 0.79)\end{array}$ & $\begin{array}{l}0.53 \\
(0.47 ; 0.60)\end{array}$ & $\begin{array}{l}0.56 \\
(0.49 ; 0.62)\end{array}$ \\
\hline
\end{tabular}

Values represent Cohen's kappa ( $\mathrm{k}$ ) or intraclass correlation coefficient (ICC) with $95 \%$ confidence interval. $\mathrm{k}$ is calculated for the presence of a cortical interruption, ICC is calculated for the total amount of cortical interruptions.

\section{Discussion}

We developed a visual scoring method to detect cortical interruptions on HR-pQCT imaging in healthy controls and patients with RA and tested its intra- and inter-reader reliability and feasibility. Cortical interruptions were seen in both healthy controls and patients with RA, but more frequently and with a larger diameter in patients with RA. We showed that most cortical interruptions (86\%) were seen in $\geq 2 x \geq 2$ slices and $21 \%$ of the cortical interruptions had adjacent trabecular distortion. Although intra-reader reliability was moderate to almost perfect, the inter-reader reliability was fair to moderate for the presence and total number of cortical interruptions. Scoring one joint took on average less than ten minutes, but the longest scoring time recorded was 38 minutes.

Different methods to investigate and interpret HR-pQCT images of mainly erosions have been proposed ${ }^{1-3,12}$. Fouque-Aubert et al. defined erosions as sharply demarcated bone lesions with juxta-articular localisation with a cortical interruption seen in at least two adjacent slices in one plane and assigned a score by percentage of bone volume 
involved (score 0-10, by 10\% volume increments) ${ }^{12}$. Stach et al. defined erosions as a clear juxta-articular interruption within the cortical shell, but did not further specify the number of adjacent slices or orthogonal planes'. Erosions were graded (grades 0-3) based on the maximum diameter of the cortical interruption. In addition, threedimensional reconstructions of the joint were made to assess cortical surface change'. Srikhum et al. defined erosions as sharply demarcated juxta-articular focal bone lesions with a cortical interruption in at least two adjacent slices in one plane, and also graded erosions (grades 0-3) based on the maximal dimension of the cortical interruption ${ }^{2}$. The SPECTRA collaboration proposed a case definition for erosion described as a cortical interruption extending over a minimum of two consecutive slices in two perpendicular planes, and measured erosion by maximum width and perpendicular to the width, the maximum depth of the erosion ${ }^{3}$. A limitation of these studies is that they specifically aimed at scoring established bone erosions in RA which, in most studies, need to be seen on two consecutive slices. As a result, only large interruptions were scored ${ }^{2}$, small cortical interruptions were not taken into account. Therefore, physiological vascular channels, which might represent starting points for erosion development ${ }^{13-15}$, were not identified. However, the strength of HR-pQCT imaging is that it has the potential to identify small (i.e. early) bone changes, also in the vascular channels. Our visual scoring method incorporates all cortical interruptions in MCP and PIP joints and hereby allows to make a distinction between small cortical interruptions and larger cortical interruptions which are considered more specific for $\mathrm{RA}^{1,3}$.

Previous studies in patients with RA showed a predilection for large cortical interruptions (erosions) at the radial quadrant ${ }^{1,16}$. Finzel et al. suggested that the palmar quadrant is the site where physiological vascular channels enter the cortex ${ }^{16}$. We observed most cortical interruptions in the palmar quadrant in healthy controls and in the palmar and radial quadrant in patients with RA. However, our method did not categorize cortical interruptions into vascular channels or erosions. We assessed adjacent trabecular distortion and observed that this was only observed in patients with RA $(21 \%)$, with the highest frequency in the ulnar quadrant. Both radial and ulnar quadrants are insertion sites of collateral ligaments and considered an area that is prone for erosion development ${ }^{17}$.

The inter-reader reliability of our scoring method was fair to moderate, however, a significant difference in the total number of cortical interruptions between the two readers was found. Several reasons for this difference were observed: smaller interruptions showed less agreement, multiple smaller interruptions were counted as one large interruption by one reader whereas the other reader counted each, and disagreement was sometimes caused by low density of the cortex. These discrepancies were also observed in a previous study from our study group, in which we used microCT as gold standard ${ }^{18}$. We found fair to substantial reliability of HR-pQCT in the detection of cortical interruptions on two consecutive slices in two planes. Other studies that tested methods for scoring bone erosions on HR-pQCT showed moderate to almost perfect reliability ${ }^{1-3}$. The lower reliability values observed in our study are most likely 
attributable to the smaller cortical interruptions which we incorporated in the visual scoring method. Reliability scores for other imaging techniques, such as conventional radiographs, also show a wide range in reliability, from moderate to almost perfect (0.47-1.00), despite being scored by experienced readers ${ }^{19}$. Visual interpretation of images remains a complex task ${ }^{20}$. Furthermore, scoring of the HR-pQCT images was time consuming with a mean time to score one joint of 9.2 minutes. Only one other study reported scoring time for MCP joints; they found a median scoring time of 2 minutes (range $1.20-5.30$ minutes) ${ }^{2}$. With a scoring time per joint of up to 38 minutes, our visual scoring method is barely feasible for clinical practise. On the other hand, with our visual scoring method, every cortical interruption can be scored, which increases the time to score per joint and hence lowers the feasibility. This might improve when scoring only interruptions with adjacent trabecular distortion, i.e. those suspected for reflecting an erosion ${ }^{1,3}$. Alternatively, automated algorithms to score the cortical interruptions could be a solution. One study has shown that a (semi) automated algorithm was highly reproducible in the detection of cortical interruptions (ICC 0.93 $(95 \% \mathrm{Cl} 0.87 ; 0.97))^{21}$.

The present study has an important limitation. The visual scoring method aimed to score cortical interruptions and to assess the presence of adjacent trabecular bone structure. The presence of adjacent trabecular bone is however an underestimation of the damage present, since totally destructed bone ends were by definition excluded from further assessment. The choice to exclude bone ends when more than $50 \%$ of the bone surface was destroyed was made based on the presence of multiple (large) indistinguishable interruptions. The goal of the visual scoring method is, however, to also detect small cortical interruptions and ultimately, to aid in making a distinction between physiological and pathological interruptions.

In conclusion, this scoring method allows detection of small cortical interruptions on HRpQCT imaging of finger joints. Although reading was time-consuming, this tool is promising for use in clinical studies. 


\section{References}

1 Stach, C. M. et al. Periarticular bone structure in rheumatoid arthritis patients and healthy individuals assessed by high-resolution computed tomography. Arthritis Rheum 62, 330-339 (2010).

Srikhum, W. et al. Quantitative and semiquantitative bone erosion assessment on high-resolution peripheral quantitative computed tomography in rheumatoid arthritis. J Rheumato/ 40, 408-416 (2013).

Barnabe, C. et al. Definition for Rheumatoid Arthritis Erosions Imaged with High Resolution Peripheral Quantitative Computed Tomography and Interreader Reliability for Detection and Measurement. J Rheumatol 43, 1935-1940 (2016).

Scharmga A, K. K., Peters M, van Tubergen A, van den Bergh J, van Rietbergen B, et al. . in Arthritis Rheumatol. 2016; 68 (supp/ 10).

Scharmga, A. et al. Visual detection of cortical breaks in hand joints: reliability and validity of highresolution peripheral quantitative CT compared to microCT. BMC Musculoskelet Disord 17, 271 (2016).

Pialat, J. B., Burghardt, A. J., Sode, M., Link, T. M. \& Majumdar, S. Visual grading of motion induced image degradation in high resolution peripheral computed tomography: impact of image quality on measures of bone density and micro-architecture. Bone 50, 111 1-1 18 (2012).

Scharmga, A. et al. Heterogeneity of Cortical Breaks in Hand Joints of Patients with Rheumatoid Arthritis and Healthy Controls Imaged by High-resolution Peripheral Quantitative Computed Tomography. J Rheumatol 43, 1914-1920 (2016).

Aletaha, D. et al. 2010 Rheumatoid arthritis classification criteria: an American College of Rheumatology/ European League Against Rheumatism collaborative initiative. Arthritis and rheumatism 62, 2569-2581 (2010). van der Heijde, D. How to read radiographs according to the Sharp/van der Heijde method. J Rheumatol 27, 261-263 (2000).

$$
\text { Barnabe, C. \& Feehan, L. High-resolution peripheral quantitative computed tomography imaging protocol }
$$
for metacarpophalangeal joints in inflammatory arthritis: the SPECTRA collaboration. J Rheumatol 39, 1494-1495 (2012).

Landis, J. R. \& Koch, G. G. The measurement of observer agreement for categorical data. Biometrics 33, 159-174 (1977). Fouque-Aubert, A. et al. Assessment of hand bone loss in rheumatoid arthritis by high-resolution peripheral quantitative CT. Ann Rheum Dis 69, 1671-1676 (2010).

Schett, G. \& Gravallese, E. Bone erosion in rheumatoid arthritis: mechanisms, diagnosis and treatment. Nat Rev Rheumatol 8, 656-664 (2012).

14 Binks, D. A. et al. Role of vascular channels as a novel mechanism for subchondral bone damage at cruciate ligament entheses in osteoarthritis and inflammatory arthritis. Ann Rheum Dis 74, 196-203 (2015). Schett, G. et al. Analysis of the kinetics of osteoclastogenesis in arthritic rats. Arthritis Rheum 52, 3192 3201 (2005).

16 Finzel, S. et al. A detailed comparative study of high-resolution ultrasound and micro-computed tomography for detection of arthritic bone erosions. Arthritis Rheum 63, 1231-1236 (2011). McGonagle, D., Tan, A. L., Moller Dohn, U., Ostergaard, M. \& Benjamin, M. Microanatomic studies to define predictive factors for the topography of periarticular erosion formation in inflammatory arthritis. Arthritis Rheum 60, 1042-1051 (2009). Scharmga, A. et al. Visual detection of cortical breaks in hand joints: reliability and validity of highresolution peripheral quantitative CT compared to microCT. BMC Musculoskelet Disord 17, 271 (2016). Sharp, J. T. et al. Variability of precision in scoring radiographic abnormalities in rheumatoid arthritis by experienced readers. J Rheumato/ 31, 1062-1072 (2004). 


\section{Supplementary files}

1. Assess image quality according to Pialat et al.* image grades 4 and 5 are excluded for further analysis.

2. Divide joint into its proximal and distal bone end, and assess joint for normal outer bone contour
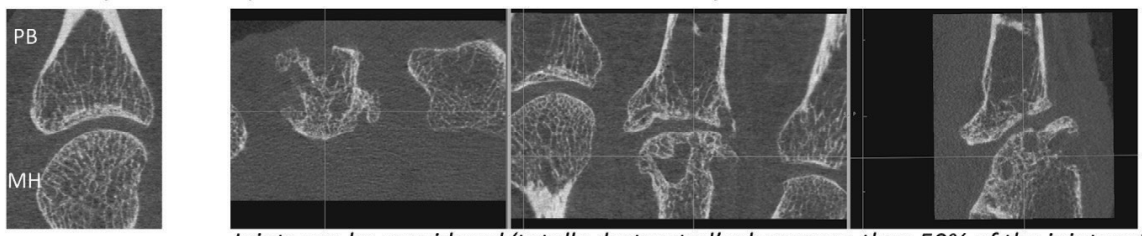

Joints can be considered 'totally destructed' when more than $50 \%$ of the joint surface is affected. There is no further evaluation of the joint in case of a totally destructed joint

3. Divide joint into four quadrants

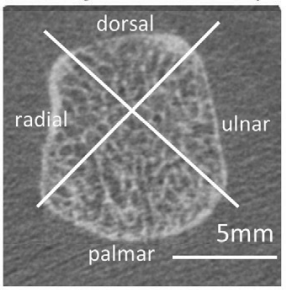

4. Check presence of cortical interruptions. A cortical interruption is scored if:

a) an interruption in the cortex is present on at least one slice in one plane, and in additional in two consecutive slices in another plane (i.e. $\geq 1 \mathrm{x} \geq 2$ or $\geq 2 x \geq 1$ ).

b) an interruption is present in more than one consecutive slice in one plane and in more than one slice in another plane (i.e. $\geq 2 x \geq 2$ and more).

5. In case of a cortical interruption, measure maximal diameter in one of three possible planes (transverse, coronal or sagittal).

6. In case of a cortical interruption, assess adjacent trabecular distortion (yes/no).
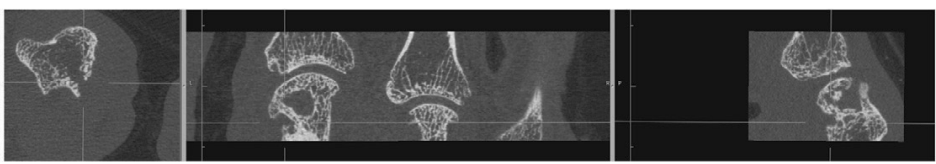

Presence of clear trabecular distortion when there is clear absence of trabeculae below the cortical interruption

* Pialat et al. 2011 Visual grading of motion induced image degradation in high resolution peripheral computed tomography: Impact of image quality on measures of bone density and micro-architecture

Supplementary figure 5.1 Steps of the visual scoring method 


\section{MOSA-Hand study}

Healthy controls $n=38$

Patients with RA $n=41$

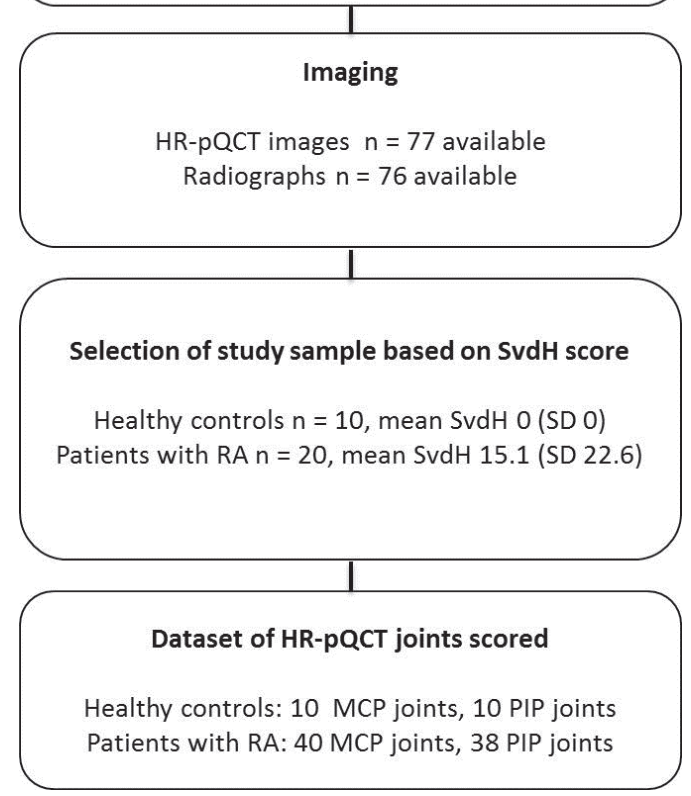

Supplementary figure 5.2 Flowchart dataset of HR-pQCT joints scored from the MOSA-Hand study Abbrevations: HR-pQCT; high resolution peripheral quantitative computed tomography, SvdH; Sharp/ van der Heijde method 
Development of a visual scoring method

transverse

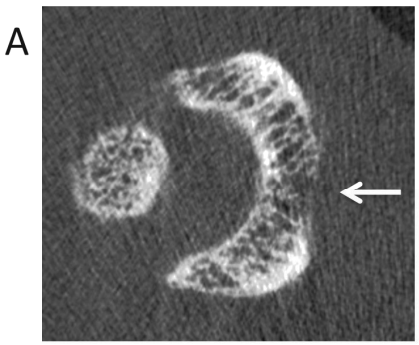

B

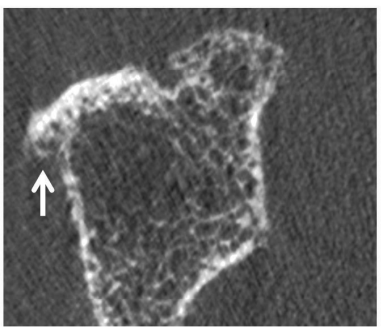

C

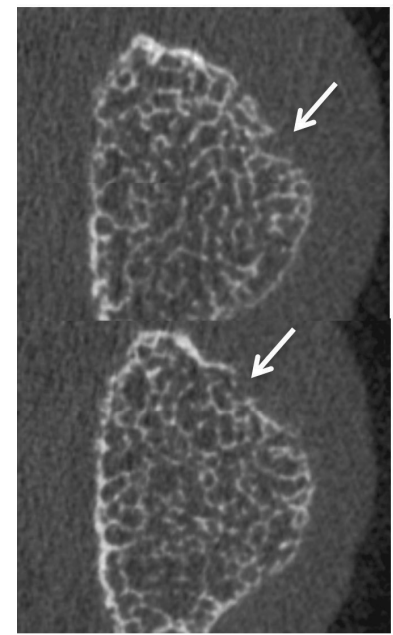

coronal
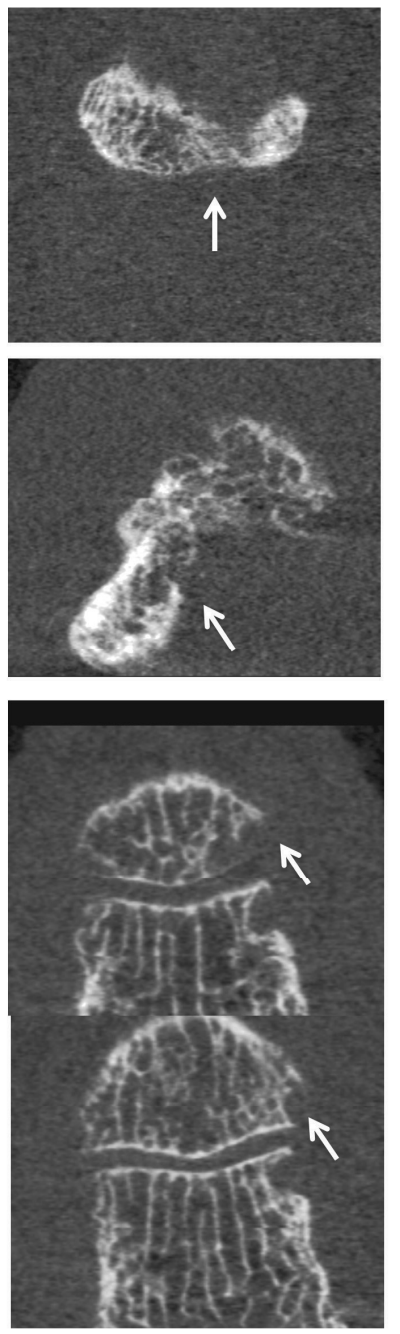

sagittal
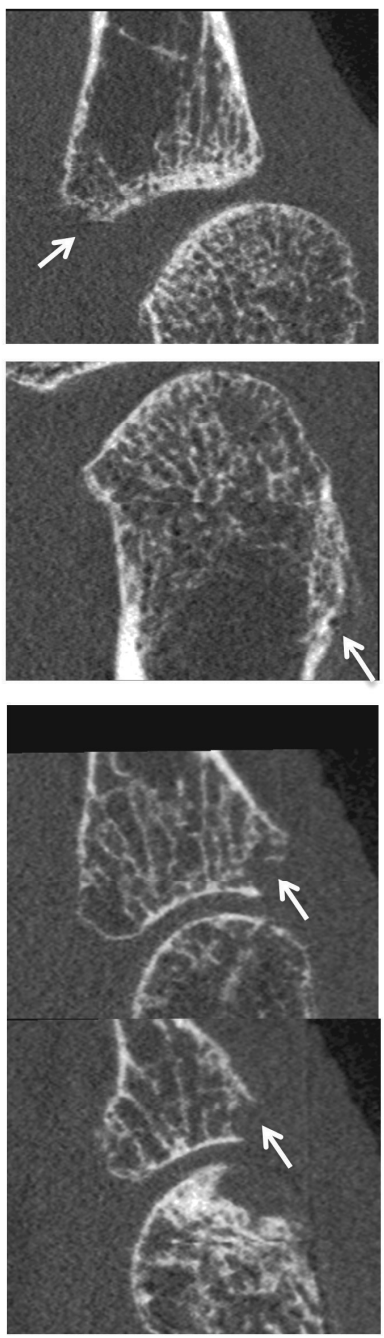

Supplementary figure 5.3 HR-pQCT images of cases leading to discrepancies between Readers in scoring

Panel A: low density caused by low mineralization and/or thin cortex

Panel B: bone apposition

Panel C: multiple breaks belonging to one large break at the base of the phalanx

Abbrevations: HR-pQCT; high resolution peripheral quantitative computed tomography

Supplementary Table 5.1. Mean (SD) number of cortical interruptions based on quadrants in healthy controls and patients with RA

\begin{tabular}{llll}
\hline & Healthy controls & Patients with RA & \\
\hline Quadrants & mean (SD) & mean (SD) & p-value \\
\hline Palmar & $0.6(0.9)$ & $0.5(0.9)$ & $\mathrm{p}=0.43$ \\
Ulnar & $0.1(0.3)$ & $0.3(0.5)$ & $\mathrm{p}=0.03$ \\
Dorsal & $0.2(0.4)$ & $0.3(0.5)$ & $\mathrm{p}=0.56$ \\
Radial & $0.1(0.3)$ & $0.5(0.6)$ & $\mathrm{p}<0.001$ \\
\hline
\end{tabular}

Values are based on results from Reader 1, first reading and calculated on quadrant level 
Structural damage and inflammation on radiographs or MRI are associated with cortical interruptions on high-resolution peripheral quantitative CT: a study in finger joints of patients with rheumatoid arthritis and healthy subjects

A. Scharmga, M. Peters, J.P. van den Bergh,

P. Geusens, D. Loeffen, B. van Rietbergen,

T. Schoonbrood, D. Vosse, R. Weijers,

A. van Tubergen

Submitted 


\begin{abstract}
Objectives. To study the relationship between structural damage and inflammatory features on either MRI or radiography and other risk factors (anti-citrullinated protein antibody (ACPA) and/or rheumatoid factor (RF) seropositivity, hand dominance, disease duration) with the presence or number of cortical interruptions in finger joints on high-resolution peripheral quantitative computed tomography (HR-pQCT).

Methods. Finger joints of 38 healthy subjects and 39 patients with RA were examined through radiographs, MRI and HR-pQCT. Radiographs were scored according to the Sharp-van der Heijde (SvdH) method, MRI for presence of cortical interruptions, bone marrow edema (BME) and synovitis, and HR-pQCT images for cortical interruptions. Descriptives were calculated, associations were examined using generalized estimating equations.
\end{abstract}

Results. Cortical interruptions were found in both healthy subjects and patients with RA on HR-pQCT (respectively mean (SD) 0.33 (0.63) versus 0.38 (0.64) per joint quadrant, $p<0.01$ ). Structural damage on either MRI (cortical interruptions) or radiographs (SvdH $\geq 1$ ) was associated with the presence of cortical interruptions on HRpQCT (respectively OR 12.4, 95\% Cl 7.5-21.4, $\mathrm{p}<0.01$ and $\mathrm{OR} 4.8,95 \% \mathrm{Cl} 1.9$ $11.7, \mathrm{p}<0.01$ ). Also, the presence of either BME or synovitis was associated with more cortical interruptions on HR-pQCT $(\mathrm{B} 0.47,95 \% \mathrm{Cl} 0.4-0.6, \mathrm{p}<0.01$ and $\mathrm{B} 1.9,95 \%$ $\mathrm{Cl}$ 0.6-3.1, $\mathrm{p}<0.01)$. In patients with RA, ACPA and/or RF seropositivity, hand dominance and disease duration were not associated with more cortical interruptions on HR-QCT.

Conclusion. Structural damage and inflammatory features on MRI and radiographs are associated with cortical interruptions on HR-pQCT. No association between other risk factors and cortical interruptions could be demonstrated. 


\section{Introduction}

Rheumatoid arthritis (RA) is an inflammatory autoimmune disease characterized by cortical interruptions of the bone. The presence, size and number of those cortical interruptions on radiographs are risk factors for poor long-term disease outcome ${ }^{1,2}$. Sensitive and reproducible imaging methods are therefore essential for the diagnosis, management and monitoring of RA. Radiographs are used as the gold standard to assess structural damage in standard clinical care. Markers of inflammation such as bone marrow edema (BME) and synovitis can be evaluated by MRI. The presence of $B M E$ on MRI has been associated with progression of damage on radiographs ${ }^{3,4}$. High resolution peripheral quantitative computed tomography (HR-pQCT) is another imaging technique used to assess cortical and trabecular bone structure in greater detail5,6. Studies to validate the use of HR-pQCT in imaging cortical interruptions in finger joints have been initiated. Comparative studies of HR-pQCT, MRI and radiography of finger joints showed that HR-pQCT is more sensitive to detect cortical interruptions compared to $M R I$ and radiographs $5,7,8$. However, these studies predominantly focused on established pathological interruptions, i.e. erosions. Studies with MRI and HR-pQCT further showed that inflammatory changes and cortical interruptions can also be found in finger joints of healthy subjects 5,9 . We demonstrated in an earlier study that cortical interruptions in finger joints detected on HR-pQCT have a heterogeneous spectrum, some of which had adjacent trabecular distortion suggestive for an erosion, and some may represent physiological vascular channels ${ }^{10}$. It is hypothesized that vascular channels might serve as a starting point for pathological cortical interruptions as they provide direct entry for osteoclast-mediated joint destruction ${ }^{11-13}$. The osteoclasts are activated by inflammation, however, in anti-citrullinated protein antibody (ACPA) positive patients with RA, bone loss was also observed in the absence of inflammation ${ }^{1,14,15}$. Besides ACPA seropositivity, other factors such as hand dominance and disease duration have also been associated with the presence and progression of structural damage in hand joints ${ }^{16-18}$. Currently, studies evaluating the relationship between structural damage and/or inflammatory features on either MRI or radiography with the presence or number of cortical interruptions in finger joints on HR-pQCT are lacking. Furthermore, it is unknown whether several other risk factors for structural damage such as ACPA and rheumatoid factor lgM (RF) seropositivity, hand dominance, and disease duration are also associated with more cortical interruptions on HR-pQCT. The aims of this study were to further validate the use of HR-pQCT by investigating the association between 1). structural damage on either MRI or radiography and the presence of cortical interruptions on HR-pQCT images of finger joints in healthy subjects and patients with RA, 2). markers of inflammation on MRI (BME and synovitis) and the number of cortical interruptions on HR-pQCT in healthy subjects and patients with RA, and 3). ACPA and RF seropositivity, hand dominance or disease duration and the number of cortical interruptions on HR-pQCT in patients with RA. 


\section{Methods}

\section{Subjects}

For this cross-sectional study, we used data from the MOSA-Hand cohort, which consists of 38 female healthy subjects and 41 female patients diagnosed with RA. All subjects were recruited at the Maastricht University Medical Center, the Netherlands. All patients with RA fulfilled the 2010 American College of Rheumatology (ACR)/European League Against Rheumatism (EULAR) classification criteria for RA ${ }^{19}$. Information on medication use, ACPA and rheumatoid factor (RF) positivity were retrieved from the patients' medical file. Healthy subjects, matched per decade, did not suffer from hand joint complaints. All subjects signed informed consent. Ethical approval was obtained from the ethics board of the academic hospital Maastricht/Maastricht University, the Netherlands. Netherlands Trial Registry number: NTR382 1.

\section{HR-pQCT image acquisition and scoring}

According to the HR-pQCT imaging protocol proposed by The Study grouP for xtrEme Computed Tomography in Rheumatoid Arthritis (SPECTRA) ${ }^{20}$, second and third metacarpophalangeal (MCP) and proximal interphalangeal (PIP) joints were scanned with HR-pQCT (XtremeCT1, Scanco Medical AG, Switzerland). Each hand was separately scanned using the standard carbon forearm cast as provided by the manufacturer. In healthy subjects only the dominant hand was scanned and in patients with RA both hands were scanned.

One experienced reader (AS) scored all HR-pQCT images according to a visual scoring algorithm for HR-pQCT as described elsewhere ${ }^{21}$. A subset of HR-pQCT images of 30 subjects was scored twice by two readers (MP and AS). Intra-reader (Reader AS) intraclass correlation coefficient (ICC) was $0.69(95 \% \mathrm{Cl} 0.65-0.73)$ per quadrant, inter -reader ICC was $0.56(95 \% \mathrm{Cl} 0.49 ; 0.62)$ per quadrant ${ }^{21}$. The scores from Reader AS were used for the present analyses.

In short, first, images were assessed for image quality according to Pialat et al. ${ }^{22}$. Second, each joint was divided into its proximal and distal bone end. When more than $50 \%$ of the joint surface of the cortical bone was destroyed, the bone end was considered 'totally destructed' and the bone end was excluded from further examination. Third, each bone end was further divided into four quadrants (palmar, ulnar, dorsal and radial). Fourth, a cortical interruption was scored when it fulfilled one of the following criteria: a). an interruption in at least one slice in one plane and in addition in two consecutive slices in another plane (i.e. $1 \times \geq 2$ or $\geq 2 \times 1$ slices), and b). an interruption in more than one consecutive slice in one plane and in more than one slice in another plane (e.g. $\geq 2 x \geq 2$ slices). Fifth, the adjacent trabecular structure was assessed for distortion. In Figure 6.1, panel 1, an example is shown of a cortical interruption in the metacarpal head on HR-pQCT. In panel 2, an example is shown of a totally destructed bone end which was excluded from further examination. 
1

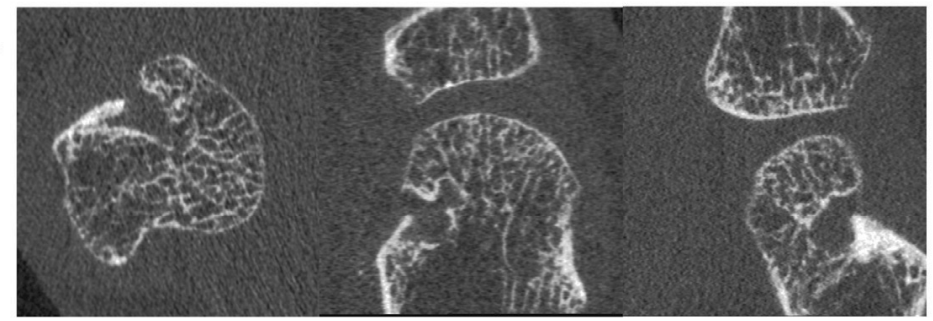

2

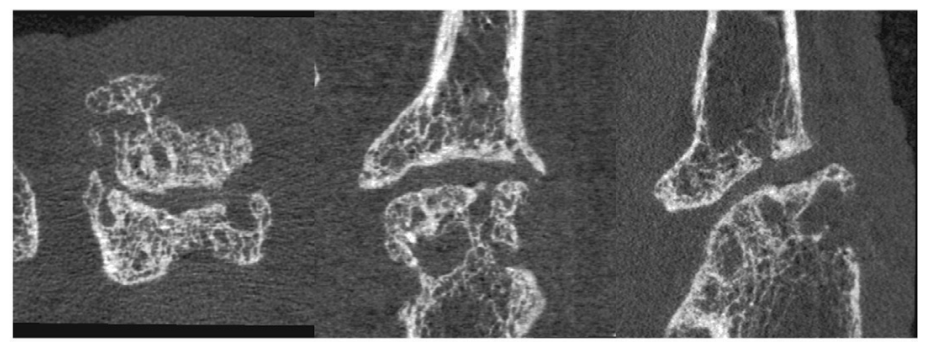

Figure 6.1. Panel 1: Example of a cortical interruption in axial, coronal and sagittal view on the radial side of a metacarpal head on HR-pQCT

Panel 2: Example of an axial, coronal and sagittal HR-pQCT image of a totally destructed bone end excluded from further examination

\section{MRI acquisition and scoring}

Second and third MCP and PIP joints of both hands were examined using a $3.0 \mathrm{~T}$ Achieva Philips MRI device. During the examination, the hand was fixed inside a dedicated wrist coil and the space around the hand was filled with rubber to reduce motion artefacts. Images were acquired of both hands using axial Tl-weighted, axial fat-suppressed T2-weighted and sagittal 3D WATSc sequences. Additional images were acquired post intravenous gadolinium (Gadovist $1.0 \mathrm{mmol} / \mathrm{ml}$ solution for injection) using axial and coronal fat-suppressed T1-weighted images.

The MRI images were independently scored by two radiologists (DL and RW), blinded for clinical data, for structural damage (the presence of cortical interruptions), and markers of inflammation (the presence of BME and synovitis). Presence of a cortical interruption and BME was evaluated per joint quadrant. Synovitis was evaluated per joint. Consensus was reached in case of discrepancies.

\section{Conventional radiography acquisition and scoring}

Posterior-anterior radiographs of the hands were taken from all subjects according to a standard clinical protocol. Two experienced and trained rheumatologists (DV and TS) independently scored the radiographs of the hands in order to assess structural bone damage according to the Sharp/Van der Heijde (SvdH) method ${ }^{23}$. Mean scores of the two readers were calculated and used for the analyses. For the present analyses, only the SvdH scores on the second and third MCP and PIP joints were taken into account. Radiographic damage was considered present when the $\mathrm{SvdH}$ score was $\geq 1$. 


\section{Statistics}

Descriptive statistics for baseline characteristics and the scores on HR-pQCT, MRI and radiographs were calculated. Differences between healthy subjects and patients with RA were calculated using Mann-Whitney $U$ tests or chi-square tests, as appropriate. Stratified analyses were done in patients with RA and analysed by Mann-Whitney $U$ test (ACPA and/or RF positive versus negative patients, $<2$ years versus $>2$ years disease duration) or Wilcoxon signed rank test (dominant versus non-dominant hand). Possible associations between structural damage, markers of inflammation, and other risk factors (ACPA and/or RF positivity, hand dominance and disease duration) with the presence or number of cortical interruptions on HR-pQCT images were tested using generalized estimating equations (GEE). An exchangeable working correlation structure was assumed in order to adjust for within-subject correlation. For the presence of structural damage, a binary model was used, providing an odds ratio (OR). For inflammatory features and other risk factors, a linear model was used, providing a beta (B) value. All GEE analyses were performed on quadrant level, except for synovitis on MRI which was performed on the joint level.

According to the visual scoring algorithm, totally destructed quadrants (when more than $50 \%$ of the bone end is totally destroyed) were excluded from evaluation on the presence of cortical interruptions, because of the indefinable boundaries, which would lead to inaccurate measures and more discrepancies between readers (see for example Figure 6.1, panel 2). Instead, we assigned a (conservative) count of "one" cortical interruption for each quadrant belonging to a totally destructed bone end. Count "one" was chosen based on the median score of the total number of cortical interruptions per affected quadrant in patients with RA (median 1, range $1-4$ per quadrant).

Statistical analyses were performed with SPSS Statistics for Windows version 23.0 (IBM Corp., Armonk, NY).

\section{Results}

Of the 38 healthy subjects and 41 patients with RA that were recruited, two patients with RA did not have a HR-pQCT scan available and were excluded from further analyses. In one subject (patient with RA) HR-pQCT images of four PIP joints were not scanned due to intolerance to long immobilization during scanning, but the remaining joints were included in the analyses. Table 6.1 shows the characteristics of the study population. Patients with RA were older compared to the healthy subjects $(p<0.01)$. An example of a normal MCP joint and an example of a totally destructed bone end visualized on corresponding radiograph, HR-pQCT images, and MRI image is shown in Figure 6.2. 
Table 6.1. Characteristics of the study population

\begin{tabular}{lll}
\hline & $\begin{array}{l}\text { Healthy subjects } \\
(\mathrm{n}=38)\end{array}$ & $\begin{array}{l}\text { Patients with RA } \\
(\mathrm{n}=39)\end{array}$ \\
\hline Age & $51.2(10.3)$ & $54.8(7.0)$ \\
Disease duration in months & $\mathrm{n} / \mathrm{a}$ & $131.7(116.6)$ \\
ACPA and/or RF positive & $\mathrm{n} / \mathrm{a}$ & $30(77 \%)$ \\
ACPA positive & $\mathrm{n} / \mathrm{a}$ & $24(59 \%)$ \\
RF positive & $\mathrm{n} / \mathrm{a}$ & $27(66 \%)$ \\
ACPA and RF positive & $\mathrm{n} / \mathrm{a}$ & $21(54 \%)$ \\
DMARD use & $\mathrm{n} / \mathrm{a}$ & $34(87 \%)$ \\
Biological use & $\mathrm{n} / \mathrm{a}$ & $20(51 \%)$ \\
DMARD and biological use & $\mathrm{n} / \mathrm{a}$ & $15(38 \%)$ \\
\hline
\end{tabular}

Values are presented as mean (SD) or as absolute number (\%)

Abbreviations: RA; rheumatoid arthritis: ACPA; anti-citrullinated protein antibody: RF; rheumatoid factor, DMARD; disease modifying antirheumatic drug, $n / a$ not applicable

In two patients, ACPA values were missing
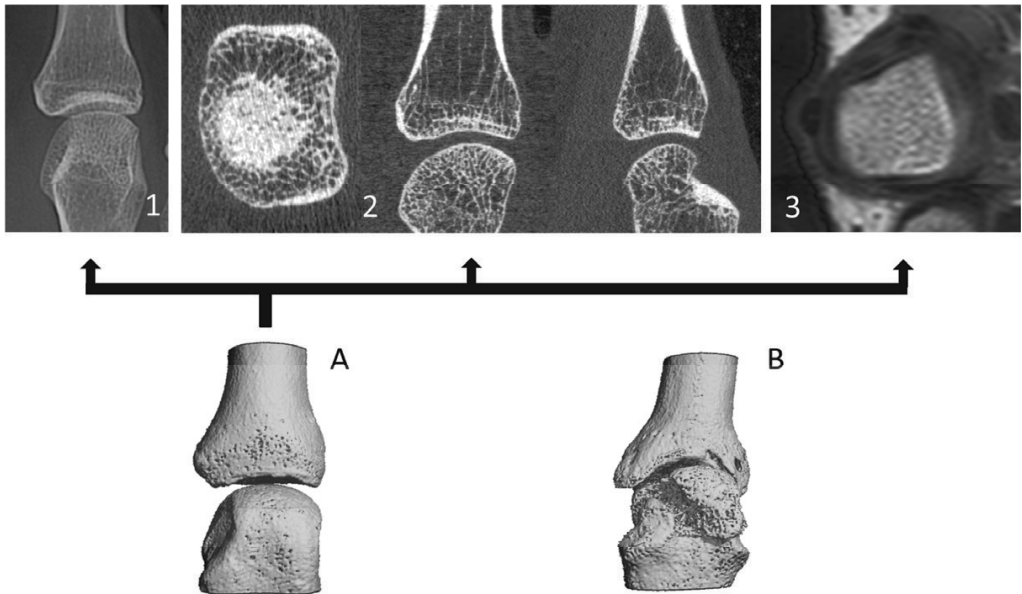

A

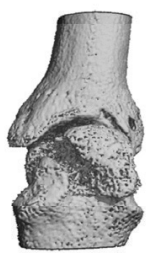

B
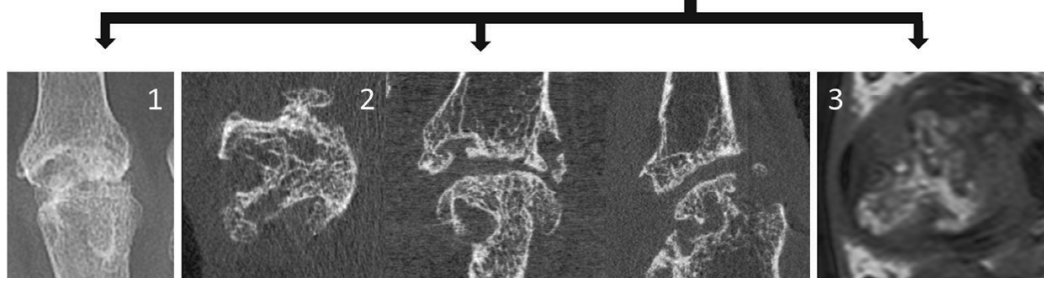

Figure 6.2. Two examples of MCP joints. Example $A$ shows an $M C P$ joint in a healthy subject with corresponding radiograph, axial, coronal and sagittal HR-pQCT images, and MRI image. Example $B$ shows a totally destructed joint in a patient with RA with corresponding radiograph, axial, coronal and sagittal HR-pQCT images, and MRI image

\section{HR-pQCT}

Table 6.2 shows the results of the HR-pQCT visual scoring method for healthy subjects and patients with RA. In total, 308 joints (2464 quadrants) were analysed in patients with RA, and 152 joints (1216 quadrants) in healthy subjects. Totally destructed bone ends were only seen in patients with RA $(n=9)$ in 76 quadrants $(3 \%$ of the quadrants 
analyzed in patients with RA). The mean number of cortical interruptions per quadrant was significantly higher in patients with RA compared to healthy subjects $(p<0.01)$. Also a higher proportion of cortical interruptions with adjacent trabecular distortion was seen in patients with RA compared to healthy subjects $(25 \%$ versus $10 \%, p<0.01)$.

Comparative stratified analyses for ACPA and/or RF positive versus negative patients with RA did not show significant differences in the mean number of cortical interruptions and adjacent trabecular distortion. Also the results from those patients who were ACPA and RF positive $(n=21)$ versus negative patients were not significantly different (data not shown).

In the dominant hand of patients with RA, cortical interruptions both with and without adjacent trabecular distortion were more frequently found compared to the nondominant hand $(p<0.01$ and $p<0.05$ respectively). Patients with $>2$ years versus $<2$ years disease duration had a significantly higher mean number of cortical interruptions per quadrant and a higher proportion of cortical interruptions with adjacent trabecular distortion (both $p<0.01$ ).

\section{MRI}

Table 6.3 shows the results of MRI scoring. Seventy-five baseline MRI scans of the 77 subjects who were imaged by HR-pQCT were available and scored 37 healthy subjects and 38 patients with RA).

In one subject, MRI images of four PIP joints were missing due to technical issues. Due to motion artefacts, twelve joints were not evaluable for BME and fourteen joints were not evaluable for cortical interruptions and synovitis. A statistically significant higher proportion of quadrants with structural damage (cortical interruptions), and inflammatory markers (BME and synovitis) was found in patients with RA compared to healthy subjects (all $p<0.01$, Table 6.3).

In ACPA and/or RF positive patients, the proportion of quadrants with BME was significantly higher compared to seronegative patients $(p<0.01$, Table 6.3). Comparable results were found for patients who were RF and ACPA positive versus negative $(p<0.01$, data not shown). In the dominant hand of patients with RA, the proportion of quadrants with BME was significantly higher compared to the nondominant hand $(p<0.01$, Table 6.3). Furthermore, in patients with $>2$ years of disease duration the proportion of quadrants with either cortical interruptions or $\mathrm{BME}$, and joints with synovitis was higher compared to patients with $<2$ years of disease duration (all $\mathrm{p}<0.05$, Table 6.3). 
Structural damage and inflammation on radiographs or MRI

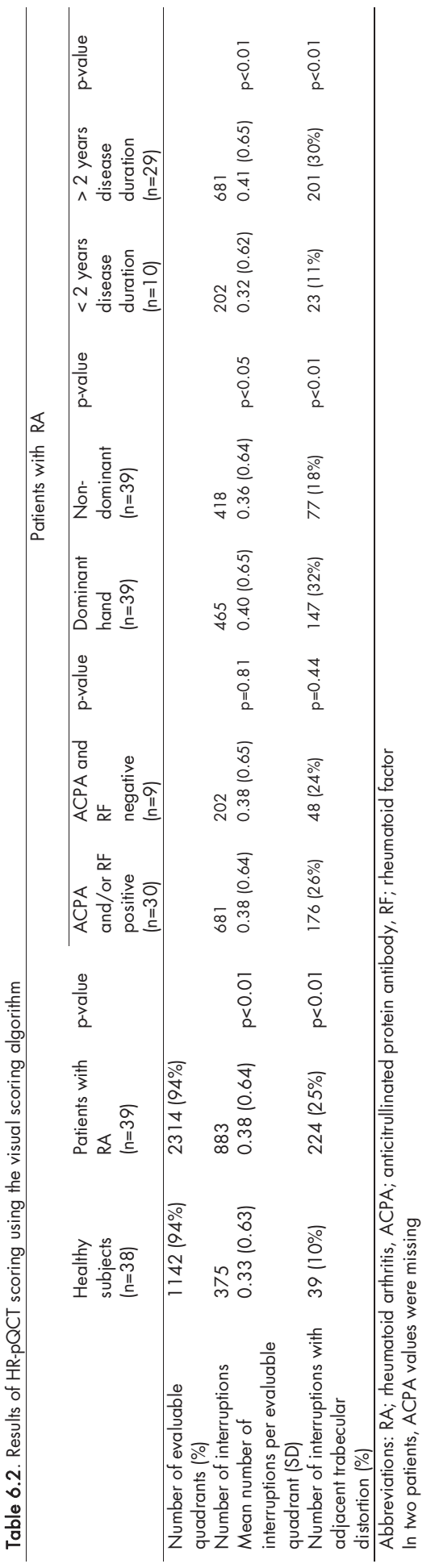




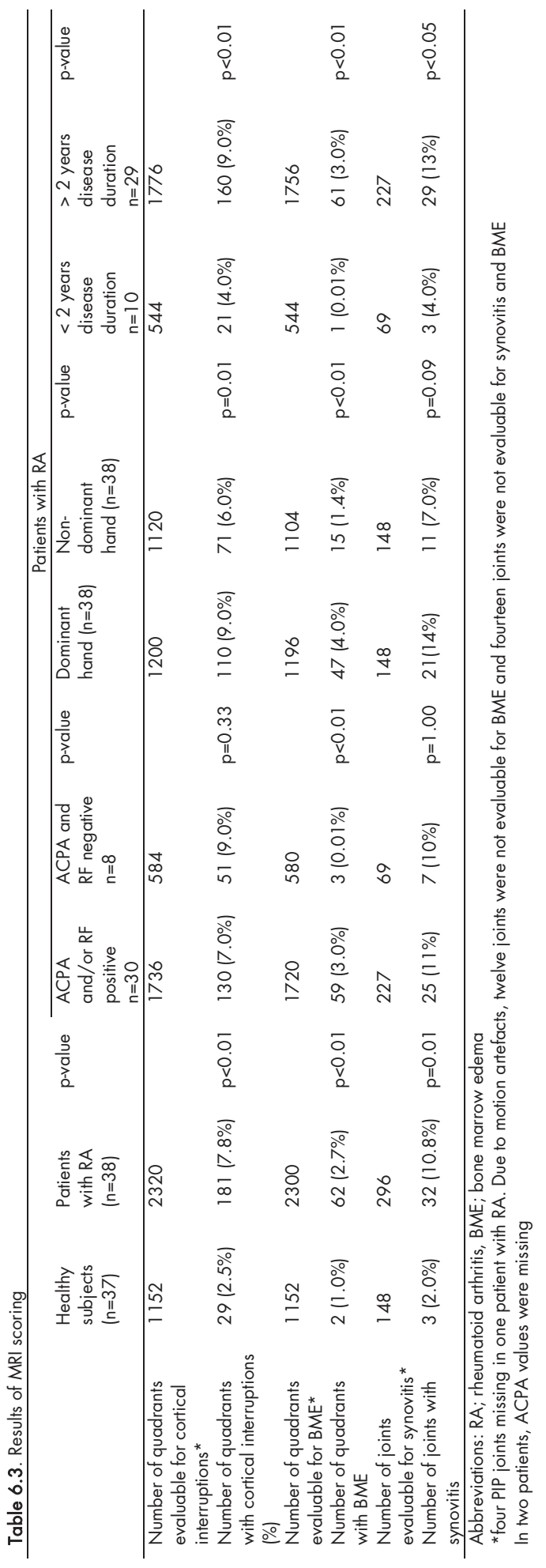




\section{Radiographs}

Seventy-five baseline radiographs of the 77 subjects with a HR-pQCT scan were available and scored (36 healthy subjects and 39 patients with RA). The mean total SvdH score based on two readers was 1.1 (SD 1.8, range 0-1) for healthy subjects and 9.1 (SD 17.3, range 0-87.5) for patients with RA. Five patients with RA showed presence of radiographic damage $(\mathrm{SvdH} \geq 1)$ in 10 joints specifically examined in this study (MCP2, 3 and PIP 2, 3 bilaterally). In the healthy subjects no radiographic damage was observed in these joints.

Association between structural damage and presence of cortical interruptions on HRpQCT

Univariable GEE analysis showed a significant association between structural damage (the presence of a cortical interruption) per quadrant on MRI and the presence of a cortical interruption per quadrant on HR-pQCT (total population: OR 12.4, 95\% Cl 7.5 - 21.4, p<0.01; patients with RA: OR 11.1, 95\% Cl 6.3 - 19.5, p<0.01). Radiographic damage (SvdH $\geq 1$ yes/no) was also associated with the presence of cortical interruptions on HR-pQCT (total population: OR 4.8, 95\% Cl $1.9-11.7$, $p<0.01$; patients with RA: OR 4.5,95\% Cl 1.8-11.7, $p<0.01$ ).

Association between markers of inflammation and number of cortical interruptions on HR-pQCT

The presence of BME was significantly associated with more cortical interruptions per quadrant on HR-pQCT (total population: B $0.5,95 \% \mathrm{Cl} 0.5-0.6, p<0.01$; patients with RA: B $0.5,95 \% \mathrm{Cl} 0.4-0.6, \mathrm{p}<0.01)$. Synovitis of the joint was also significantly associated with more cortical interruptions on HR-pQCT (total population: B 1.9, 95\% $\mathrm{Cl} 0.6-3.1, \mathrm{p}<0.01$, patients with RA: B 2.0, 95\% $0.7-3.4, \mathrm{p}<0.01$ ).

Association between other risk factors and the number of cortical interruptions on HRpQCT

In patients with RA, the presence of ACPA and/or RF was not associated with more cortical interruptions per quadrant on HR-pQCT (B 0.0,95\% Cl -0.1 - 0.1, p=0.94). Also hand dominance and disease duration did not show a significant association with the number of cortical interruptions per quadrant on HR-pQCT $(B) 0.0,95 \% \mathrm{Cl}-0.0-$ $0.1, \mathrm{p}=0.21$ and $\mathrm{B} 0.1,95 \% \mathrm{Cl} 0.0-0.2, \mathrm{p}=0.06$ respectively).

\section{Discussion}

This paper aimed to study associations between structural damage, markers of inflammation and other risk factors on the presence and number of cortical interruptions on HR-pQCT. Cortical interruptions, with or without adjacent trabecular distortion were seen in both healthy subjects and patients with RA on HR-pQCT, but more frequently in patients with RA. Structural damage on MRI or radiographs was significantly associated with the presence of cortical interruptions on HR-pQCT. When BME and synovitis were 
present, significantly more cortical interruptions were found on HR-pQCT. In patients with RA, ACPA and/or RF seropositivity, hand dominance and disease duration were not associated with the number of cortical interruptions on HR-pQCT.

Previous studies showed that the presence of BME on MRI is associated with cortical interruptions and progression of structural damage on radiographs ${ }^{3,4,24}$. Also, BME and synovitis in the joints in a healthy, symptom-free, study population have been observed on MRI $(57.5 \% \text { and } 48.2 \% \text {, respectively) })^{9}$. In our study, we also found BME in both patients with RA and healthy subjects. When BME was present, more cortical interruptions on HR-pQCT were found, both in the total population, including healthy subjects, and in patients with RA.

A clear relationship between the presence of ACPA and/or RF and more radiographic damage has been reported ${ }^{25}$. Using quantitative analyses, Kleyer et al. showed significant bone loss in finger joints on HR-pQCT before the clinical onset of RA in ACPA positive subjects without signs of arthritis compared to ACPA negative subjects ${ }^{15}$. In the present study, we were unable to demonstrate more cortical interruptions on HRPQCT in patients who are ACPA and/or RF positive. This could be explained by lack of statistical power due to a low number of patients in the ACPA and RF negative group $(n=9)$, but also by the fact that all patients included in this study received treatment, of which more than $50 \%$ a biological. In a study using HR-pQCT, repair of large cortical interruptions detected in patients with RA after treatment with tumor necrosis factor inhibitors has been demonstrated ${ }^{26}$.

To date, only limited research on structural damage between dominant and nondominant hand has been performed, showing more structural damage in the dominant hand ${ }^{16,18}$. In a crude comparative analysis, we found significantly more cortical interruptions per quadrant on HR-pQCT in the dominant hand versus the non-dominant hand. Because the presence of structural damage is associated with more damage in the same subject ${ }^{27}$, we corrected for this within subject correlation by using GEE analyses, after which the association could no longer be demonstrated. The same was observed with disease duration, which showed a significant difference in the crude comparative analyses, but in the GEE analyses, no association was found.

The strength of the present study is that we performed it both in patients with RA and in healthy subjects, and we were able to compare findings on three different imaging modalities in the same patient. An important limitation is that the visual scoring method for HR-pQCT takes into account all cortical interruptions and does not distinguish between physiological vascular channels or pathological erosions, which might be the reason why many cortical interruptions were found in healthy subjects. However, it is hypothesized that vascular channels could be the starting point for pathological cortical interruptions ${ }^{1-13}$. Werner et al. recently showed the presence of so-called cortical microchannels in a specific region on the joint; the bare area, an area considered prone to development of cortical interruptions. Patients with RA showed more cortical microchannels at a younger age compared to healthy individuals at higher age ${ }^{28}$. Longitudinal studies are needed to explore the role of these physiological cortical 
interruptions and/or cortical micro-channels in the development of pathological cortical interruptions.

In conclusion, structural damage and inflammatory features on radiographs and MRI are associated with more cortical interruptions on HR-pQCT. An association between other risk factors and more cortical interruptions could not be demonstrated. 


\section{References}

1 Gravallese, E. M. \& Walsh, N. C. Rheumatoid arthritis: Repair of erosion in RA-shifting the balance to formation. Nat Rev Rheumatol 7, 626-628 (2011).

2 Lillegraven, S., van der Heiide, D., Uhlig, T., Kvien, T. K. \& Haavardsholm, E. A. What is the clinical relevance of erosions and joint space narrowing in RA? Nat Rev Rheumatol 8, 117-120 (2012). Gandjbakhch, F., Foltz, V., Mallet, A., Bourgeois, P. \& Fautrel, B. Bone marrow oedema predicts structural progression in a 1-year follow-up of 85 patients with RA in remission or with low disease activity with lowfield MRI. Ann Rheum Dis 70, $2159-2162$ (2011).

Hetland, M. L. et al. MRI bone oedema is the strongest predictor of subsequent radiographic progression in early rheumatoid arthritis. Results from a 2 -year randomised controlled trial (CIMESTRA). Ann Rheum Dis 68, 384-390 (2009).

Stach, C. M. et al. Periarticular bone structure in rheumatoid arthritis patients and healthy individuals assessed by high-resolution computed tomography. Arthritis Rheum 62, 330-339 (2010).

Barnabe, C. et al. Definition for Rheumatoid Arthritis Erosions Imaged with High Resolution Peripheral Quantitative Computed Tomography and Interreader Reliability for Detection and Measurement. J Rheumatol 43, 1935-1940 (2016).

Regensburger, A. et al. A comparative analysis of magnetic resonance imaging and high-resolution peripheral quantitative computed tomography of the hand for the detection of erosion repair in rheumatoid arthritis. Rheumatology (Oxford) 54, 1573-1581 (2015).

Lee, C. H. et al. Correlation of structural abnormalities of the wrist and metacarpophalangeal joints evaluated by high-resolution peripheral quantitative computed tomography, 3 Tesla magnetic resonance imaging and conventional radiographs in rheumatoid arthritis. Int J Rheum Dis 18, 628-639 (2015). Mangnus, L., van Steenbergen, H. W., Reiinierse, M. \& van der Helm-van Mil, A. H. Magnetic Resonance Imaging-Detected Features of Inflammation and Erosions in Symptom-Free Persons From the General Population. Arthritis Rheumatol 68, 2593-2602 (2016). Scharmga, A et al. Heterogeneity of Cortical Breaks in Hand Joints of Patients with Rheumatoid Arthritis and Healthy Controls Imaged by High-resolution Peripheral Quantitative Computed Tomography. J Rheumatol 43, 1914-1920 (2016). Schett, G. \& Gravallese, E. Bone erosion in rheumatoid arthritis: mechanisms, diagnosis and treatment. Nat Rev Rheumatol 8, 656-664 (2012).

Binks, D. A. et al. Role of vascular channels as a novel mechanism for subchondral bone damage at cruciate ligament entheses in osteoarthritis and inflammatory arthritis. Ann Rheum Dis 74, 196-203 (2015). Schett, G. et al. Analysis of the kinetics of osteoclastogenesis in arthritic rats. Arthritis Rheum 52, 3192 3201 (2005). Paleolog, E. M. The vasculature in rheumatoid arthritis: cause or consequence? Int J Exp Pathol 90, 249 261 (2009).

Kleyer, A. et al. Bone loss before the clinical onset of rheumatoid arthritis in subjects with anticitrullinated protein antibodies. Ann Rheum Dis (2013).

Koh, J. H. et al. Radiographic Structural Damage Is Worse in the Dominant than the Non-Dominant Hand in Individuals with Early Rheumatoid Arthritis. PLoS One 10, e0135409 (2015). Srikhum, W. et al. Quantitative and semiquantitative bone erosion assessment on high-resolution peripheral quantitative computed tomography in rheumatoid arthritis. J Rheumatol 40, 408-4 16 (2013). Owsianik, W. D., Kundi, A., Whitehead, J. N., Kraag, G. R. \& Goldsmith, C. Radiological articular involvement in the dominant hand in rheumatoid arthritis. Ann Rheum Dis 39, 508-510 (1980).

Aletaha, D. et al. 2010 Rheumatoid arthritis classification criteria: an American College of Rheumatology/ European League Against Rheumatism collaborative initiative. Arthritis and rheumatism 62, 2569-258 1 (2010).

Barnabe, C. \& Feehan, L. High-resolution peripheral quantitative computed tomography imaging protocol for metacarpophalangeal joints in inflammatory arthritis: the SPECTRA collaboration. J Rheumatol 39, 1494-1495 (2012). Resolution peripheral Quantitative Computed Tomography in rheumatoid arthritis and healthy controls. Submitted.

Pialat, J. B., Burghardt, A. J., Sode, M., Link, T. M. \& Majumdar, S. Visual grading of motion induced image degradation in high resolution peripheral computed tomography: impact of image quality on measures of bone density and micro-architecture. Bone 50, 111 -1 18 (2012). 27, 261-263 (2000).

A, Boyesen, $P$, Ostergaard M , Schildvold, A \& Kvien, T, K. Magnetic resonance imaging findings in 84 patients with early rheumatoid arthritis: bone marrow oedema predicts erosive progression. Ann Rheum Dis 67, 794-800 (2008).

Courvoisier, N. et al. Prognostic factors of 10-year radi
prospective study. Arthritis Res Ther 10, R106 (2008).

26 Finzel, S. et al. Repair of bone erosions in rheumatoid arthritis treated with tumour necrosis factor inhibitors is based on bone apposition at the base of the erosion. Ann Rheum Dis 70, 1587-1593 (2011). van der Heijde, D. Radiographic progression in rheumatoid arthritis: does it reflect outcome? Does it reflect 
Structural damage and inflammation on radiographs or MRI

treatment? Ann Rheum Dis 60 Suppl 3, iii47-50 (2001).

Werner, D. et al. Rheumatoid arthritis is characterized by early changes of the cortical micro-channel (CoMiC) system in the bare area of the joints. Arthritis Rheumatol (2017). 
An automated algorithm for the detection of cortical interruptions on high-resolution peripheral quantitative computed tomography images of finger joints

M. Peters, A. Scharmga, J. de Jong,

A. van Tubergen, P. Geusens, J. J. Arts, D. Loeffen,

R. Weijers, B. van Rietbergen, J. van den Bergh

PLoS One. 2017 Apr 20;12(4) 


\begin{abstract}
Objectives: To introduce a fully-automated algorithm for the detection of small cortical interruptions $(\geq 0.246 \mathrm{~mm}$ in diameter) on high resolution peripheral quantitative computed tomography (HR-pQCT) images, and to investigate the additional value of manual correction of the automatically obtained contours (semi-automated procedure).
\end{abstract}

Methods: Ten metacarpophalangeal joints from seven patients with rheumatoid arthritis (RA) and three healthy controls were imaged with HR-pQCT. The images were evaluated by an algorithm according to the fully-and semi-automated procedure for the number and surface of interruptions per joint. Reliability between the fully- and semiautomated procedure and between two independent operators was tested using intraclass correlation coefficient (ICC) and the proportion of matching interruptions. Validity of single interruptions detected was tested by comparing it to visual scoring, as gold standard. The positive predictive value (PPV) and sensitivity were calculated.

Results: The median number of interruptions per joint was 14 (range 2 to 59) and did not significantly differ between the fully-and semi-automated procedure $(p=0.37)$. The median interruption surface per joint was significantly higher with the fully- vs. semiautomated procedure (respectively, $8.6 \mathrm{~mm}^{2}$ vs. $5.8 \mathrm{~mm}^{2}$ and $6.1 \mathrm{~mm}^{2}, p=0.01$ ). Reliability was almost perfect between the fully- and semi-automated procedure for both the number and surface of interruptions $(I C C \geq 0.95)$ and the proportion of matching interruptions was high $(\geq 76 \%)$. Also the inter-operator reliability was almost perfect (ICC $\geq 0.97$, proportion of matching interruptions $82 \%$ ). The PPV ranged from $27.6 \%$ to $29.9 \%$, and sensitivity from $69.7 \%$ to $76.3 \%$. Most interruptions detected with the algorithm, did show an interruption on a 2D grayscale image. However, this interruption did not meet the criteria of an interruption with visual scoring.

Conclusion: The algorithm for HR-pQCT images detects cortical interruptions, and its interruption surface. Reliability and validity was comparable for the fully- and semiautomated procedures. However, we advise the use of the semi-automated procedure to assure quality. The algorithm is a promising tool for a sensitive and objective assessment of cortical interruptions in finger joints assessed by HR-pQCT. 


\section{Introduction}

Peri-articular cortical interruptions are one of the characteristic features of bone involvement in rheumatoid arthritis $(R A)^{1,2}$. They may occur already at an early stage and are predictors of further radiographic progression ${ }^{3}$. The presence, number, and size of interruptions within a joint and the number of joints affected are associated with a poor functional outcome $2,4,5$.

Conventional radiographs (CR) are considered the gold standard for detection of cortical interruptions in hand joints in RA. However, CR has its limitations since the sensitivity of the detection of cortical interruptions is low compared with other imaging modalities such as Computed Tomography (CT), magnetic resonance imaging (MRI) and Ultrasound (US) 6-9. High resolution peripheral quantitative CT (HR-pQCT) is a lowdose imaging technique that is able to assess the three-dimensional (3D) bone structure of the peripheral skeleton at the micro-scale $(82 \mu \mathrm{m}$ isotropic voxel size) in vivo. Studies with HR-pQCT in finger joints showed higher sensitivity for the detection of cortical interruptions compared with US, CR and MRI ${ }^{10-13}$.

Cortical interruptions, together with changes in the underlying trabecular bone architecture, such as absence of trabeculae, with or without surrounding sclerosis, are a hallmark of bone erosions. Several studies reported results on the visual inspection of the presence, number and size of cortical interruptions as part of analyzing erosions using HR-pQCT10-20. High intra- and inter-rater reliability for the visual scoring of the number of erosions was found, with intra-class correlation coefficients (ICC) and kappa values ranging from 0.6 to $1.0^{10-12,18}$. However, in all studies only established erosions and thus relatively large cortical interruptions were scored (mean width $>2 \mathrm{~mm}^{15,21}$, mean depth $>2 \mathrm{~mm}^{15,21}$, mean maximal dimension $>3 \mathrm{~mm}^{11}$ and mean volume $>4 \mathrm{~mm}^{3}$ $14,19)$. Studies that systematically investigate the visual detection of smaller cortical interruptions on HR-pQCT images are currently lacking, but it is known that this is a laborious, complex and difficult process ${ }^{22}$. Although these small interruptions might not be specific for $\mathrm{RA}^{10}$, they might be prone to bone resorption early in the course of RA and can therefore be the first signs of erosions ${ }^{23}$. Moreover, it has been shown that the number of these small interruptions is increased in patients with more active disease, suggesting a link to synovitis ${ }^{10}$.

Automated algorithms are an alternative to visual scoring of cortical interruptions on HRPQCT images. Recently, an algorithm has been developed that accurately measured erosion volume and erosion surface area, but still required an operator to visually identify the erosion and manually set a seeding point in the erosion ${ }^{19}$. A fully-automated detection method of (small) cortical interruptions was not yet available.

Here, we introduce a newly developed, fully-automated algorithm for the detection of small cortical interruptions ( $\geq 0.246 \mathrm{~mm}$ in diameter) on HR-pQCT images. Because the algorithm might be susceptible for errors in the contouring, we investigated the additional value of correction of the contours by an operator (semi-automated procedure) as suggested by Burghardt et al. ${ }^{24}$. In addition, we tested the validity of the cortical interruptions detected by the algorithm compared to visual scoring, as gold 
standard.

\section{Materials and Methods}

\section{Patients}

We selected a convenient sample of seven patients with RA (mean age 53.4 (SD 7.3) years, mean disease duration 8.2 (SD 9.8) years) and three healthy controls (HCs) (mean age 48.0 (SD 7.8) years) from the observational MOSA-Hand cohort study, including 41 patients with RA and $38 \mathrm{HCs}$ at the Maastricht University Medical Center, the Netherlands. The subjects were considered eligible when the HR-pQCT scan was of sufficient quality ( $\leq$ grade 3 , as described by Pialat et al. ${ }^{25}$ ), and the joint was not destroyed. All patients with RA fulfilled the 2010 American College of Rheumatology (ACR)/European League Against Rheumatism (EULAR) classification criteria for RA ${ }^{26}$. None of the HCs suffered from hand joint complaints. Ethical approval was obtained from the ethics board of the academic hospital Maastricht, the Netherlands (NL42300.068.12 / METC 12-2-037). All participants signed informed consent.

\section{HR-pQCT scanning procedure}

MCP joints of both hands in patients with RA and the dominant hand in HCs were scanned with HR-pQCT (XtremeCT1, Scanco Medical AG, Switzerland). The scanning was performed at clinical in vivo settings, i.e. at $60 \mathrm{kVp}$ tube voltage, $900 \mu \mathrm{A}$ tube current, $100 \mathrm{~ms}$ integration time and $82 \mu \mathrm{m}$ isotropic voxel size. The reference line was placed on top of the second metacarpal head, such that the scan region covered a length of $16.56 \mathrm{~mm}$ in proximal direction and $10.50 \mathrm{~mm}$ in distal direction (total scan length $27.06 \mathrm{~mm}, 330$ slices). The total scanning time was approximately 9 minutes and the effective dose was $<9 \mu \mathrm{Sv}$. In each patient, the third MCP joint of the first scan that was available of a patient (either left or right sided) was selected for this study.

\section{Cortical interruption detection algorithm}

We developed the automated algorithm in several steps. In order to reduce the influence of noise on the images, we considered discontinuities in the cortex as cortical interruptions only if there was an opening of at least 5 voxels through the cortex and connected to both the periosteal and endosteal boundary of the cortical mask. This corresponds to a discontinuity in at least 3 consecutive slices and a width of at least 3 voxels $(=0.246 \mathrm{~mm})$. In addition, a cortical mask with a constant thickness of 4 voxels $(=0.328 \mathrm{~mm})$ was chosen because this approached the average cortical thickness in the MCP joints in this study, which was $0.39 \mathrm{~mm}$. We decided to set the thickness of the cortical mask lower, because the thickness of the cortex at the rim of the joint, where we expected most cortical interruptions, is in general thinner ${ }^{14}$. The minimal intra-cortical interruption volume that can be detected by our algorithm is 20 voxels $\left(0.011 \mathrm{~mm}^{3}\right)$, based on an opening of at least 5 voxels * depth of 4 voxels. The 4 steps of the algorithm are explained below and displayed in Figures 7.1 and 7.2, and supplementary figure 7.1 . 

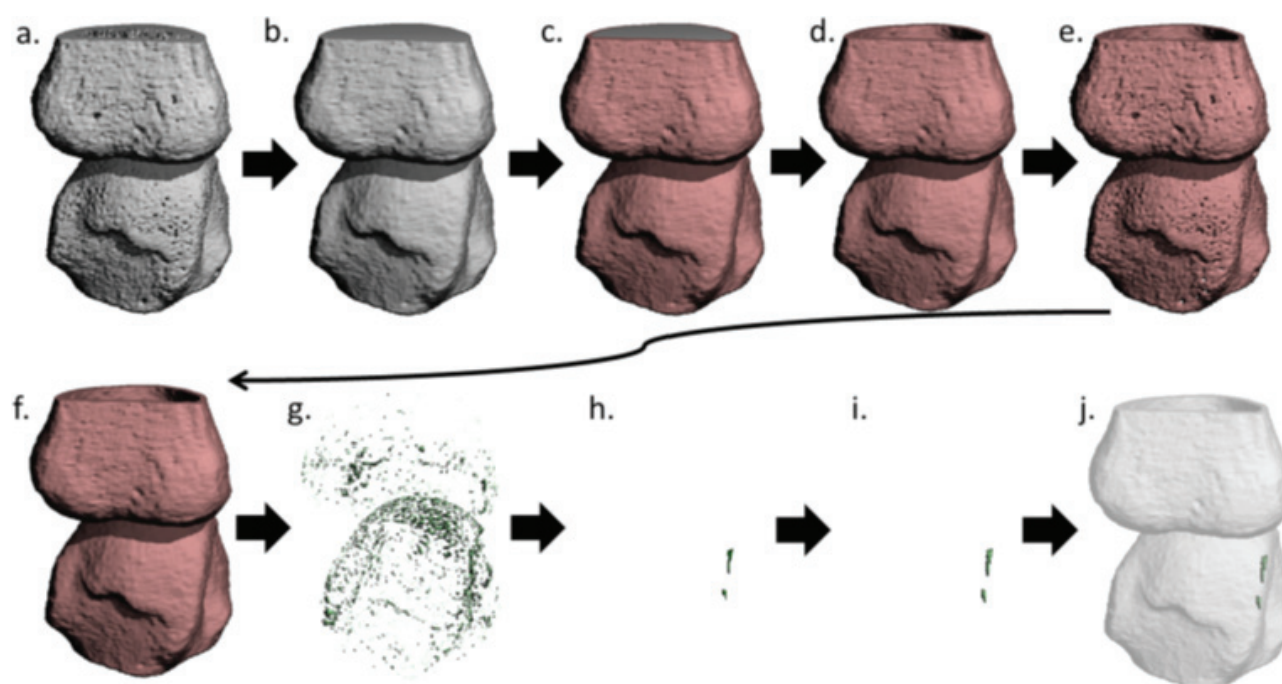

g.

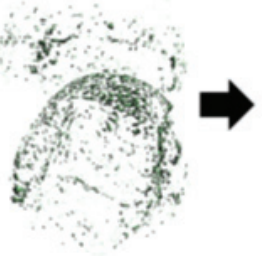

h.

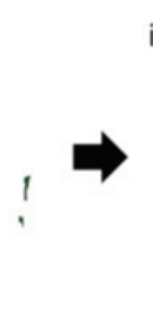

i.

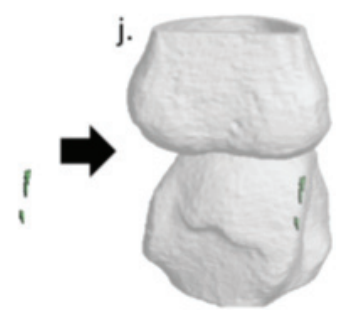

Figure 7.1. Representation of the steps executed by the algorithm. Representation of the steps executed by the algorithm applied to a 3D reconstruction of a HR-pQCT image of an MCP joint. Based on the outer margin contour of the original bone structure (a.) a solid volume is created (b.). The outer shell of this volume is segmented and depicted in red (c.) using an erosion operation. This is the cortical mask (d.), which is used to identify the cortical bone (e.). This cortical bone is dilated to fill small cavities (f.). Next, the image is inverted (g.) and only interruptions that were connected to the endosteal and periosteal boundary are selected (h.). The remaining cortical interruptions are dilated to their original volume (i.) and the results can be visually inspected by adding a transparent cortical mask (i.).

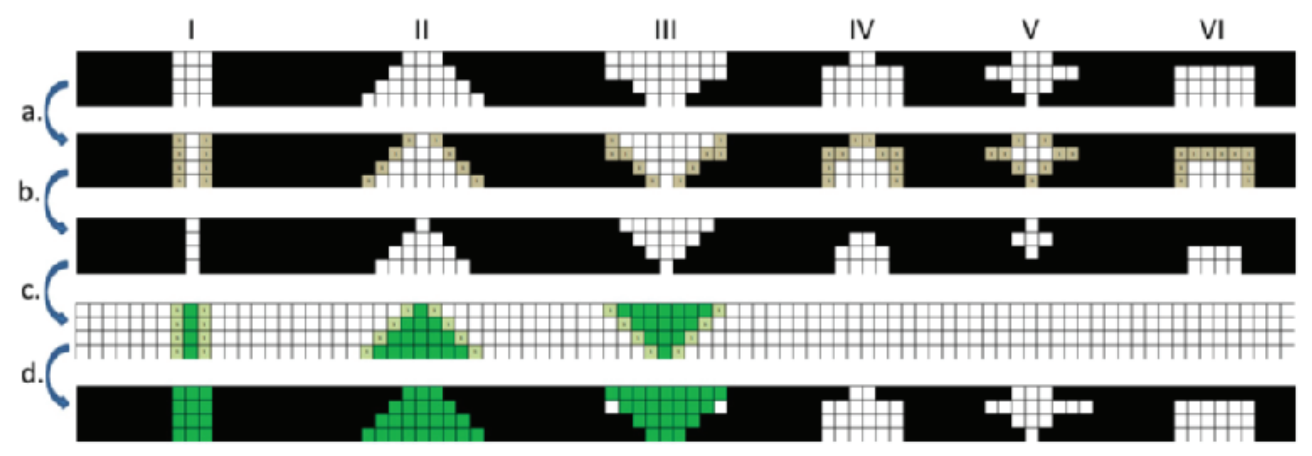

Figure 7.2. 2D examples of cortical interruptions on voxel level that are detected and are not detected by the algorithm. 2D examples of cortical interruptions on voxel level that are detected (I-III) and are not detected (IV-VI) by the algorithm. The following steps are made by the algorithm: The original cortex (step a, depicted in black) is dilated with 1 voxel (step b, depicted in grey). The cortex is then inverted (step c), only interruptions that are connected with the endosteal and periosteal boundary are selected and dilated to (approximately) its original size. The interruptions that are detected are subsequently displayed in the cortex (green) (d). Interruptions that are not connected to both the endosteal and periosteal boundary (IV-VI) with at least 3 pixels are not detected by the algorithm. 
First, the outer contour of the cortex is identified. We applied a modified version of the auto-contouring algorithm developed for periosteal segmentation of the distal radius and tibia ${ }^{24,27}$. Modifications on this algorithm were made for the structure approximation of the bone, which was performed using Gaussian filtering (sigma=0.8, support= 1 voxel) with a constant threshold of 105 instead of 120 per 1000 of the maximum possible voxel value, because the cortex in finger joint is thinner and lower mineralized compared to radius and tibia in order to reduce the effect of partial volumes. In addition, and in contrast, with the originally developed algorithm, 7 instead of 10 closing steps (dilation followed by erosion in 3D) were performed, because finger joints are smaller compared to radius and tibia. This algorithm is explained in supplementary figure 7.1 .

Second, a binary 3D model is created by extracting the voxels that contain mineralized bone using the standard evaluation protocol from the manufacturer for radii and tibia, which includes Laplace-Hamming filtering and thresholding ${ }^{28}$. An example of a binary 3D model of an MCP joint is shown in Figure 7.1 a.

Third, a cortical mask is generated by creating a 3D binary solid volume based on the outer margin contour (Figure 7.1 b). This volume is then eroded in 3D by a distance of 4 voxels. The eroded volume is subtracted from its original volume, resulting in the outer shell of the contoured volume with a constant thickness of 4 voxels (Figure 7.1c). This shell is used as the mask (Figure 7.1d) to identify the cortical bone (Figure 7.1e).

Fourth, the cortical bone is analyzed for discontinuities that meet the preset criteria of a cortical interruption (i.e. a connectivity of 5 voxels through the cortex and to both the periosteal and endosteal boundary of the cortical mask). First, the bone within the cortical mask is dilated in 3D by a distance of 1 voxel (Figure 7.1 $\mathrm{f}$ ). Second, the image is inverted (Figure 7.1g), and only interruptions that remain connected to both the endosteal and periosteal boundaries of the cortical mask are selected for further image processing (Figure 7.1 h). These interruptions are dilated again in 3D by 1 voxel to restore the original volume (Figure 7.1i) and are considered cortical interruptions. Based on this analysis, an image can be created with a transparent cortical mask as reference (Figure 7.1 j). Subsequently, the total number of cortical interruptions and the intra-cortical volume of each cortical interruption is determined using a function that identifies unconnected structural components and their volume. The interruption surface is calculated by dividing the intra-cortical interruption volume by the thickness of the cortical mask $(=0.328 \mathrm{~mm})$. Figure 7.2 shows $2 \mathrm{D}$ examples of cortical interruptions on voxel level, which are detected and not detected by the algorithm. Only interruptions on at least 3 consecutive slices and a width of at least 3 voxels through the cortex are detected. The algorithm is enclosed as supplementary file.

\section{Correction of the contours}

All HR-pQCT images were also evaluated by the semi-automated procedure in which the contour generated in step 1 of the fully-automated procedure is visually inspected and, if necessary, corrected by an operator as recommended by Burghardt et al. ${ }^{24}$. To 
test the inter-operator reliability, this correction was performed by two operators independently.

\section{Visual scoring}

All HR-pQCT images were scored visually on cortical interruptions for comparison with the algorithm. A cortical interruption was defined as a clear interruption of the cortex, seen on two consecutive slices on two orthogonal planes (on transverse, and on sagittal or coronal plane) ${ }^{29,30}$.

\section{Statistical analysis}

In this study, the results of the fully-automated procedure (AUTO), the two operators (OP1 and OP2) of the semi-automated procedure, and visual scoring were compared. Descriptives of the number of interruptions and the interruption surface were calculated. Friedman test was used for comparison across the operators and visual scoring. Posthoc analyses were performed using Wilcoxon signed-rank test between the operators, and between the operators and visual scoring.

Reliability between AUTO and the semi-automated procedure, and between OPI and OP2 for the number of interruptions and interruption surface on the joint level was estimated by ICCs with a two-way random model and absolute agreement. In addition, the reliability on the joint level between the algorithm (AUTO, OP1 and OP2) and visual scoring for the number of interruptions was obtained using ICCs.

Reliability was rated according to Landis et al.: $<0.00$ poor, $0.00-0.20$ slight, 0.21 0.40 fair, 0.41-0.60 moderate, 0.61-0.80 substantial, 0.81-1.00 almost perfect ${ }^{31}$. In addition, reliability was evaluated by the proportion of matching interruptions across the operators. Interruptions were counted as matching interruptions if they overlapped with at least 20 voxels $\left(0.011 \mathrm{~mm}^{3}\right)$, because this is the smallest intra-cortical interruption volume that could be detected with this algorithm. The proportion of matching interruptions was calculated for the presence (yes/no) of a cortical interruption on exactly the same location according to equation 1. Moreover, the validity of the algorithm to detect single interruptions on exactly the same location was evaluated by calculation of the positive predictive value (PPV, equation 2), and sensitivity (equation 3) using the visual scoring as gold standard. Statistical analysis was performed using IBM SPSS Statistics for Windows, Version 20.0 (IBM Corp., Armonk, NY). 
Proportion of matching interruptions $=$

nr. of matching interruptions $\mathrm{op}_{12}$

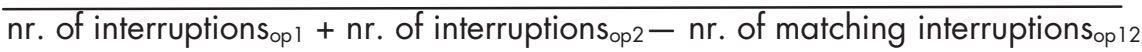

(1)

$\mathrm{Nr}$. of interruptionsop1: Number of interruptions detected by $1^{\text {st }}$ operator

$\mathrm{Nr}$. of interruptionsop2: Number of interruptions detected by $2^{\text {nd }}$ operator

$\mathrm{Nr}$. of matching interruptionsop12: Number of matching interruptions between $1^{\text {st }}$ and $2^{\text {nd }}$ operator

$\mathrm{PPV}=\frac{\mathrm{nr} \text {. of matching interruptions }}{\mathrm{nr} . \text { of interruptions } \mathrm{algorithm}} * 100 \%$

(2)

PPV: Positive predictive value

$\mathrm{Nr}$. of interruptionsalgorithm: Number of interruptions detected by the algorithm

$\mathrm{Nr}$. of matching interruptions: Number of matching interruptions between the algorithm and visual scoring

Sensitivity $=\frac{\mathrm{nr} \text {. of matching interruptions }}{\mathrm{nr} . \text { of interruptions } \text { visual scoring }} * 100 \%$

(3)

Nr. of interruptionsvisual scoring: Number of interruptions detected with visual scoring

$\mathrm{Nr}$. of matching interruptions: Number of matching interruptions between the algorithm and visual scoring

\section{Results}

Visual impression

Output algorithm

Both the fully-and semi-automated procedures detected multiple cortical interruptions in $\mathrm{HCs}$ and patients with RA (Figure 7.3). Figure 7.3 shows a typical example of 3D reconstructions of an MCP joint of an $\mathrm{HC}(I)$, a patient with early RA $(<2$ years since diagnosis) (II), and a patient with late RA (>10 years since diagnosis) (III), and the detected interruptions of the fully-automated procedure. Multiple cortical interruptions (depicted in green) can be seen in the 3D images and most interruptions are located at the rim of the joint (Figure 7.3a). Figure 7.3b shows corresponding 2D grayscale images of examples of interruptions that were detected by the fully-automated as well as the semi-automated procedure. 

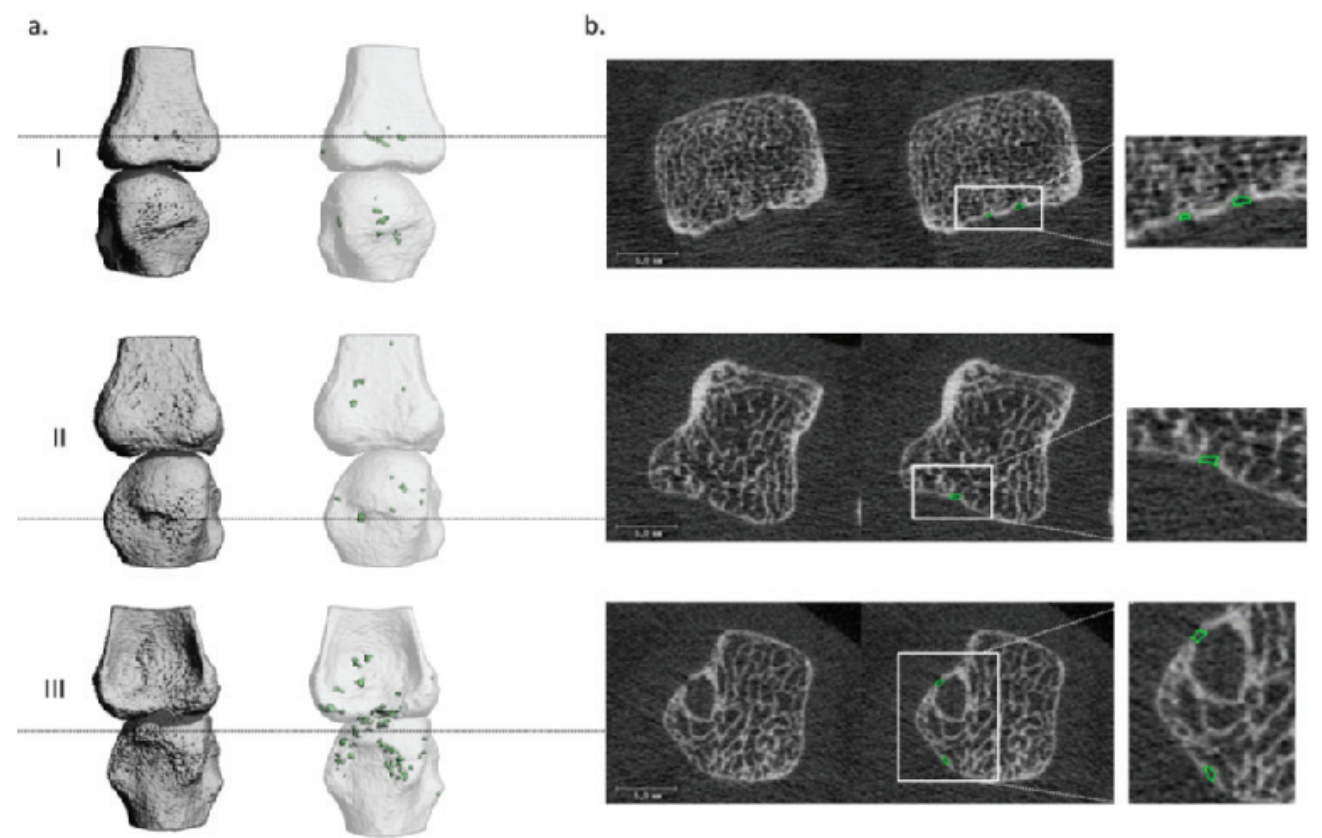

Figure 7.3. Examples of the $2 D$ and $3 D$ outputs of the algorithm. Typical examples of a 3D reconstruction of an MCP joint for a HC (I), a patient with early RA (<2 years since diagnosis) (III), and a patient with late RA (>10 years since diagnosis) (III), with the corresponding 3D outputs of the algorithm. The cortical region is indicated in transparent white. Cortical interruptions of $\geq 0.246 \mathrm{~mm}$ that are detected by the algorithm are shown in green. b) Corresponding 2D grayscale images with in green cortical interruptions detected by the algorithm.

\section{Correction of the contours}

Figure 7.4 shows examples of contours obtained with the fully-automated procedure that were manually corrected by the operators. In two cases, large cortical interruptions were corrected by both operators. For small cortical interruptions (diameter $<1 \mathrm{~mm}$ ) no correction was necessary by the operators. Figure 7.4a.I shows an example of a large cortical interruption (diameter $>1.0 \mathrm{~mm}$, white arrow). The contour obtained with the fully -automated procedure did not follow the outer margin of the original structure at the location of the cortical interruption (Figure 7.4a.II). Both operators corrected the contour (Figure 7.4a.III, white arrow) to more precisely detect the size of the large cortical interruption (Figure 7.4a.IV). Fig 4b.I shows an example of a small motion artifact (white arrow). Due to this motion artifact, the contour obtained with the fully-automated procedure was not tight around the original structure (Figure 7.4b.II), which led to a false detection of an interruption (Figure 7.4b.IV). Both operators corrected the contour (Figure 7.4b.IV). 

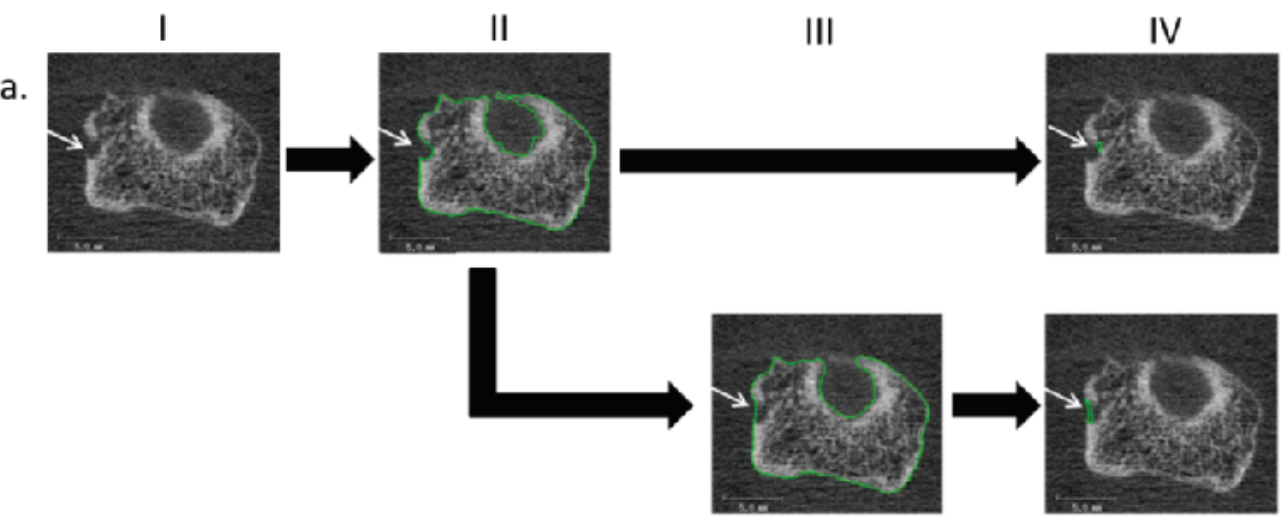

b.
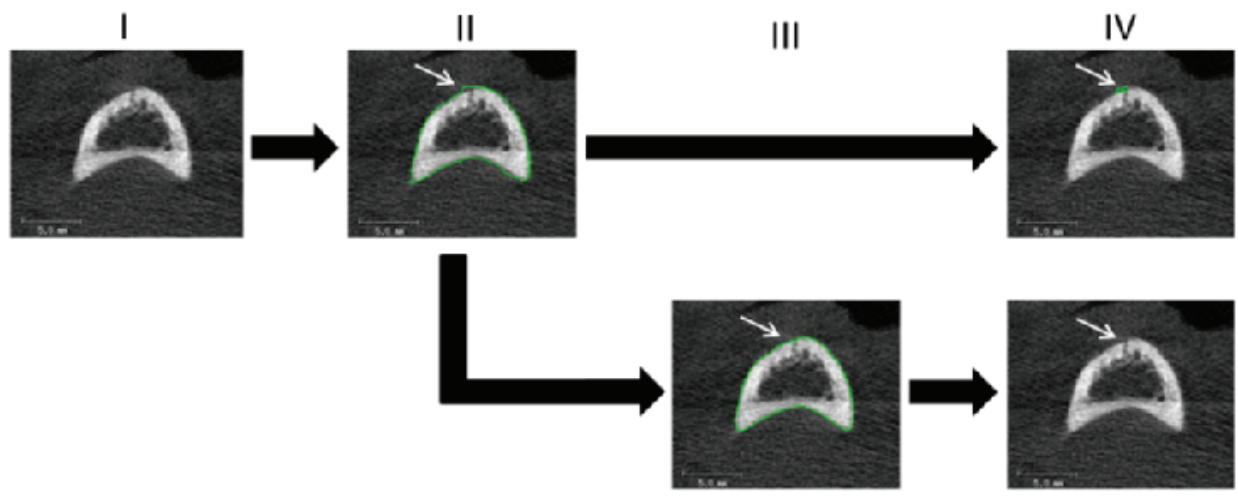

Figure 7.4. Examples of contours that were manually corrected. Typical examples of 2D grayscale images in which the contour is manually corrected by the operators. In a) a large cortical interruption is shown (a. I, arrow). The automatically obtained contour does not follow the outer margin of the original structure at the cortical interruption (a. II). The operators therefore corrected the contour (a. III) to accurately detect the large cortical interruption (IV). In b) a small motion artifact is shown (I). Due to this motion artifact, the automatically obtained contour was not tight around its original structure (III). The operators corrected this (III) and therefore no interruption was detected (IV).

\section{Quantitative comparison}

Table 7.1 shows the number of interruptions and interruption surface detected by the algorithm (AUTO, OP1 and OP2), and visual scoring. The median number of interruptions was 14 and ranged from 2 to 59 interruptions per joint for the algorithm when including all operators. The median number of interruptions with visual scoring was 5 and ranged from 3 to 22 interruptions per joint. Statistical significant differences were found across the algorithm and visual scoring for the number of interruptions $(p<0.01)$. The post-hoc analysis showed significant differences between the algorithm (AUTO, OP1 and OP2) versus visual scoring (all, $p \leq 0.01$ ).

The median of the interruption surface per joint was $7.0 \mathrm{~mm}^{2}$ and ranged from $1.5 \mathrm{~mm}^{2}$ to $76.7 \mathrm{~mm}^{2}$ when including all operators. The average surface of a single interruption 
was $<1.0 \mathrm{~mm}^{2}$. The interruption surface per joint was significantly lower with the semiautomated vs. the fully-automated procedure (Table 7.1). The post-hoc analysis showed a significant difference between AUTO and OP1 ( $p=0.02)$, but no significant difference between AUTO and OP2 ( $p=0.07)$, and OP1 and OP2 (p=0.29).

The reliability between AUTO and both OP1 and OP2 was almost perfect for both the total number of interruptions and the interruption surface (ICC $\geq 0.95, p<0.01)$. The majority of interruptions were detected on exactly the same location $(\geq 76.6 \%)$ (Table 7.2). The inter-operator reliability was also almost perfect (ICC $\geq 0.97, p<0.01$ ), as was the proportion of matching interruptions $(82.0 \%)$.

Table 7.1. The number of cortical interruptions and interruption surface per joint detected by the algorithm and with visual scoring

\begin{tabular}{|c|c|c|c|c|c|}
\hline & \multicolumn{3}{|c|}{ Algorithm } & \multirow[t]{2}{*}{ Visual } & \multirow[b]{2}{*}{ p-value * } \\
\hline & AUTO & OPI & OP2 & & \\
\hline $\begin{array}{l}\text { Number of } \\
\text { Interruptions }\end{array}$ & $\begin{array}{c}15.0^{\$} \\
{[6-49]}\end{array}$ & $\begin{array}{c}13.5^{\$} \\
{[2-59]}\end{array}$ & $\begin{array}{c}14.0^{\$} \\
{[3-51]}\end{array}$ & $\begin{array}{c}5.0^{\$} \\
{[3-22]}\end{array}$ & $<0.01$ \\
\hline $\begin{array}{l}\text { Interruption surface } \\
\left(\mathrm{mm}^{2}\right)\end{array}$ & $\begin{array}{c}8.6^{\#} \\
{[2.3-67.7]}\end{array}$ & $\begin{array}{c}5.8^{\#} \\
{[1.5-68.9]}\end{array}$ & $\begin{array}{c}6.1 \\
{[2.6-76.7]}\end{array}$ & & 0.02 \\
\hline
\end{tabular}

Values are displayed as: median [min - max]

AUTO = fully-automated procedure

$\mathrm{OP} 1$ = semi-automated procedure manual correction by operator 1

OP2 = semi-automated procedure manual correction by operator 2

* p-values obtained from Friedman test

$\$ p \leq 0.01$ obtained from post-hoc Wilcoxon signed-rank test (algorithm (AUTO, OP1 and OP2) vs. visual)

$\# p<0.05$ obtained from post-hoc Wilcoxon signed-rank test (AUTO vs. OP 1)

Table 7.2. Reliability of the algorithm.

\begin{tabular}{|c|c|c|c|}
\hline & AUTO vs. OPl & AUTO vs. OP2 & Inter-operator \\
\hline Number of Interruptions & $\begin{array}{l}\text { ICC } 0.96 \\
(95 \% \mathrm{Cl} 0.87-0.99)\end{array}$ & $\begin{array}{l}\text { ICC } 0.95 \\
(95 \% \mathrm{Cl} 0.83-0.99)\end{array}$ & $\begin{array}{l}\text { ICC } 0.97 \\
(95 \% \text { CI } 0.90-0.99)\end{array}$ \\
\hline Interruption Surface & $\begin{array}{l}\text { ICC } 0.98 \\
(95 \% \mathrm{Cl} 0.77-1.00)\end{array}$ & $\begin{array}{l}\text { ICC } 0.96 \\
(95 \% \mathrm{CI} 0.85-0.99)\end{array}$ & $\begin{array}{l}\text { ICC } 0.98 \\
(95 \% \mathrm{Cl} 0.92-1.00)\end{array}$ \\
\hline Proportion of matching Interruptions & $81.1 \%$ & $76.6 \%$ & $82.0 \%$ \\
\hline \multicolumn{4}{|c|}{$\begin{array}{l}\text { Reliability of the fully-automated vs. semi-automated (AUTO vs. OP I and AUTO vs. OP2) and semi-automated i } \\
\text { (OP1 vs. OP2) of the algorithm is shown. ICCs are calculated on the total number of cortical interruptions and } \\
\text { interruption surface in all joints. Proportion of matching interruptions is calculated on the presence of a } \\
\text { cortical interruption (yes/no) on exactly the same location. ICC = intra-class correlation coefficient, } \\
95 \% \mathrm{Cl}=95 \% \text { confidence interval }\end{array}$} \\
\hline
\end{tabular}

\section{Comparison to visual scoring}

The reliability between the algorithm (AUTO, OP1 and OP2), and visual scoring was fair to moderate for the number of interruptions (ICC ranging from 0.38 to 0.45 ). The PPV of interruptions detected by the algorithm compared to visual scoring was fair (PPV ranging from $27.6 \%$ to $29.9 \%$ ). The sensitivity of the algorithm compared to visual scoring was substantial (sensitivity ranging from $69.7 \%$ to $76.3 \%$ ). Examples of interruptions detected visually and with the algorithm are shown in Figure 6.5. An example of an interruption detected visually as well as with the algorithm is shown (Figure 6.5.I). Figure 6.5.II shows interruptions detected with the algorithm which did 

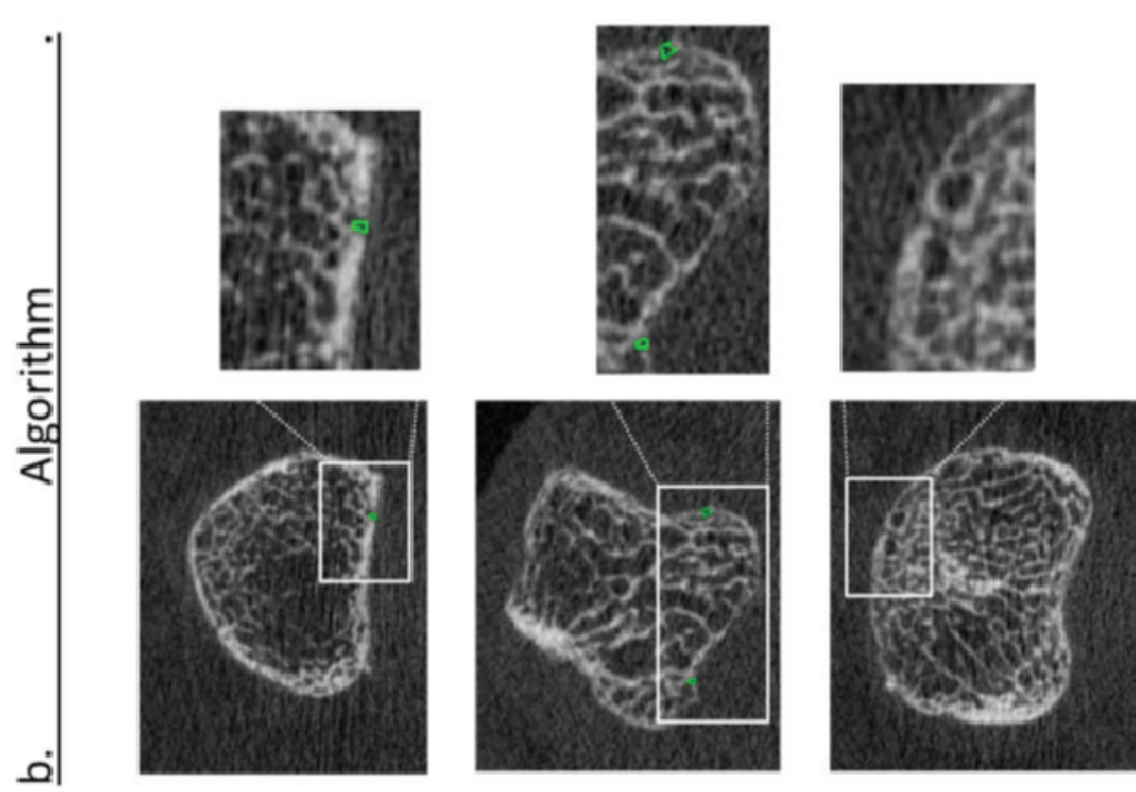

능

는 듕

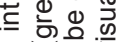

할을

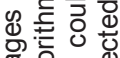

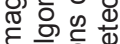

뜽응

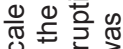

化 3

ते

कᄒ

읗은 은

ब 3 융

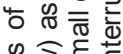

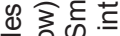

है 品的宁

एक

零完

त్

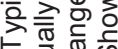

क 흐

至文

흔

品过品
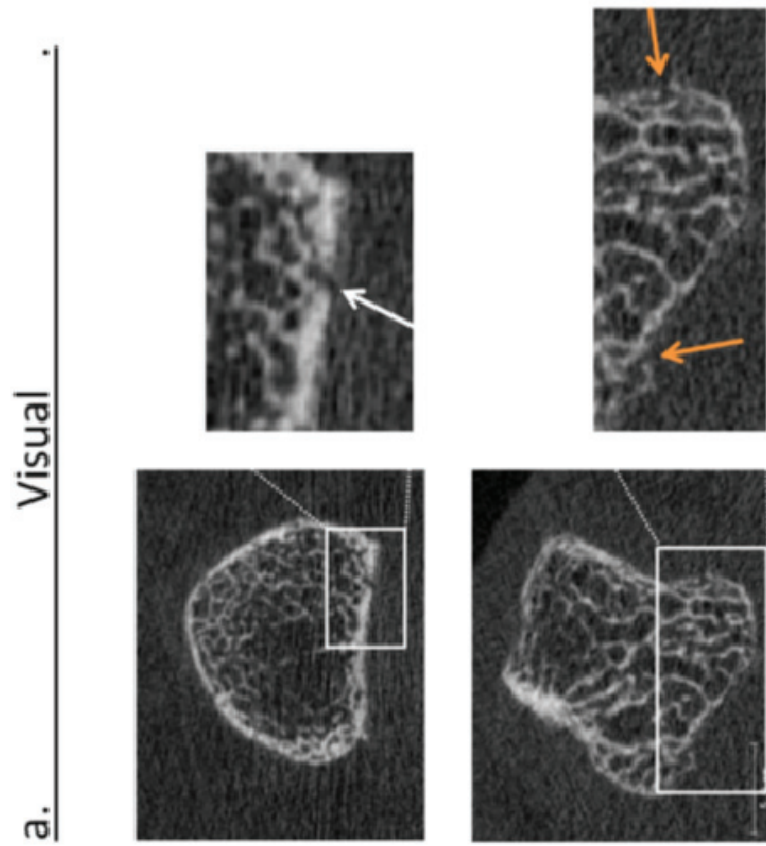

웅엉이

들웡

3 능

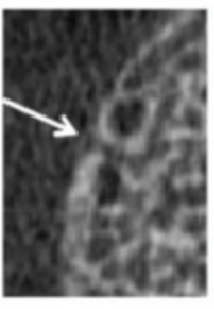

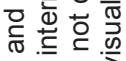

\宁边

需的乡

. $>$ क $\frac{0}{3}$.

월흘

过 就

o 흠

की
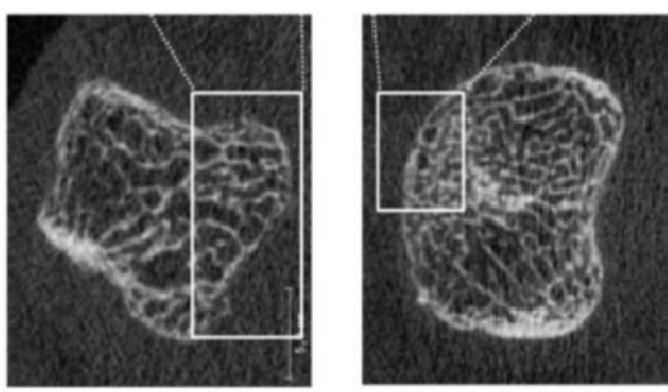

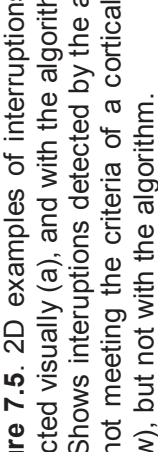

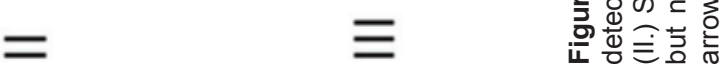


not meet with the criteria of visual scoring. Figure 6.5.III shows an interruption that was detected visually, but missed by the algorithm.

\section{Discussion}

In this study, we introduced a fully-automated algorithm to detect small cortical interruptions in HR-pQCT images and tested the additional value of correction by an operator. In addition, we tested the validity of interruptions detected by the algorithm by comparing it to visual scoring, as gold standard. The interruption surface detected with the fully-automated procedure was significantly higher compared with the semiautomated procedure, but the absolute number of interruptions did not significantly differ. Reliability was almost perfect between both procedures (ICC $\geq 0.95$ ) meaning that the manual correction of the contours was of little additional value. In addition, the proportion of matching interruptions on exactly the same location was substantial to almost perfect across the operators $(\geq 76.6 \%)$. Lastly, the validity of a single interruption was similar for both procedures. We found that most interruptions detected by the algorithm were not identified as a cortical interruption with the visual scoring (70-72\%). However, in most cases an interruption was present, but did not met the preset criteria (Figure 7.5.II). In addition, most interruptions detected visually were detected by the algorithm (70-76\%). However, it remains important that the images are rated on motion artifacts before applying the fully-automated algorithm. Only a scan quality with grade $\leq 3$ according to Pialat et al. ${ }^{25}$ should be included in the analysis. Even small motion artifacts may lead to false positive detection of cortical interruptions as exemplified in this study (Figure 7.4b). Compared to visual scoring of the number of cortical interruptions ${ }^{10-19}$, our approach has the advantage of providing an automated and objective measure of evaluation of cortical interruptions and its interruption surface, including small interruptions.

The number of cortical interruptions per joint detected in our study (median 14, ranging from 2 to 59) is substantially higher than observed in other studies using HR-pQCT, ranging from 0.5 to 2.9 interruptions per MCP joint $10,11,15,16,18,19,21$. This can mainly be explained by the fact that our algorithm detects smaller cortical interruptions (mean interruption surface $<1 \mathrm{~mm}^{2}$, equivalent to a width of $<1.13 \mathrm{~mm}$ when assuming a circle) compared with a visual scoring of the number of interruptions (mean width $>2 \mathrm{~mm}^{15,21}$ ). Furthermore, in these studies the number of interruptions was scored on 2D grayscale images whereas our automated algorithm detects interruptions on 3D binary images, which has the advantage of having an objective manner to distinguish between bone and non-bone. The use of binary images has been validated earlier in human tibia and radius 32,33 . A potential disadvantage of binary images is the partial volume effect, which mainly affects regions where the cortical bone is thin, i.e. close to the image spatial resolution, leading to the misperception that an interruption is present in the binary image. We showed examples of interruptions that were detected with the algorithm displayed in 2D grayscale images (Figures 7.3 and 7.5). In most cases, a clear interruption in the cortex can be seen, whereas in some cases an interruption was 
detected which could not be seen on the 2D grayscale images, as a result of the partial volume effect (Figures 7.3 and 7.5). This potential error might be resolved when using the second generation HR-pQCT (HR-pQCT 2). The HR-pQCT 2 has a higher spatial resolution compared to the HR-pQCT (95 $\mu \mathrm{m}$ versus $130 \mu \mathrm{m}$, respectively). Thus, the HRpQCT 2 might be able to detect these thin structures, which may reduce the number of interruptions detected as a result of the partial volume effect. However, with the HRpQCT 2, the cortical mask thickness should be increased to 5 voxels $(=0.305 \mathrm{~mm})$, to obtain a similar cortical mask thickness as for the first generation HR-pQCT. In addition, the minimum diameter of interruptions is smaller on the HR-pQCT 2 due to the smaller voxel size $(\geq 0.183 \mathrm{~mm}$ versus $\geq 0.246 \mathrm{~mm})$. Hence, the HR-pQCT 2 is able to detect even smaller interruptions, which might lead to a higher number of interruptions detected. The fully-automated differed from the semi-automated procedure in the detection of large cortical interruptions (diameter $>1 \mathrm{~mm}$ ). In the first step of the fullyautomated algorithm, where the outer contour of the bone is identified, the original outer contour of the finger joint may not be restored in the case of large interruptions. In the example of Figures $7.4 a$, both operators manually corrected this. For small cortical interruptions (diameter $<1 \mathrm{~mm}$ ) no correction was necessary. The role for HR-pQCT will mainly be for the detection of small cortical interruptions, as larger interruptions are already visible on other imaging techniques. Although not specific for $\mathrm{RA}^{10}{ }^{10}$, the small interruptions might be prone to bone resorption early in the course of RA ${ }^{23}$. Clinical follow-up studies in patients with RA are needed to investigate whether these small interruptions may become established erosions.

Some limitations of the study should be mentioned. First, we included a small number of subjects, however, a large number of cortical interruptions (199 in total) was detected in these joints, which provided us reliable estimates for the proportion of matching interruptions. Second, the cortical mask was set to a constant thickness because this would avoid any inaccuracies in the detection of the endosteal cortical bone contour. A disadvantage of our approach is that in relatively thick cortical regions, that are located more distal from the joint, cortical interruptions can be detected which are not connected to the endosteal cortical bone surface, as was exemplified in Fig 7.4b. In addition, the interruption surface may be overestimated in thin cortical regions, because a small part of the trabecular region is considered as part of the interruption.

As RA is characterized by the development of cortical interruptions in hand joints, we expect that this fully-automated algorithm can help in the assessment of small cortical interruptions in finger joints by HR-pQCT. The algorithm needs to be further validated in clinical studies in order to obtain its potential value.

In conclusion, the algorithm presented in this study allows the detection of cortical interruptions, and its interruption surface. Reliability and validity was comparable for the fully- and semi-automated procedures. However, we advise the use of the semiautomated procedure to assure quality. We expect that this algorithm is a promising tool for a sensitive and objective assessment of cortical interruptions in finger joints by HR-pQCT. 


\section{References}

1 Schett, G. \& Gravallese, E. Bone erosion in rheumatoid arthritis: mechanisms, diagnosis and treatment. Nat Rev Rheumatol 8, 656-664 (2012).

Geusens, P. \& van den Bergh, J. Bone erosions in rheumatoid arthritis. Rheumatology (Oxford) 53, 4-5 (2014).

Hulsmans, H. M. et al. The course of radiologic damage during the first six years of rheumatoid arthritis. Arthritis and rheumatism 43, 1927-1940 (2000).

van der Heijde, D. M. et al. Prognostic factors for radiographic damage and physical disability in early rheumatoid arthritis. A prospective follow-up study of 147 patients. British journal of rheumatology 31, 519 -525 (1992).

Lillegraven, S., van der Heijde, D., Uhlig, T., Kvien, T. K. \& Haavardsholm, E. A. What is the clinical relevance of erosions and joint space narrowing in RA? Nat Rev Rheumato/ 8, 117-120 (2012).

Ejbjerg, B. et al. Magnetic resonance imaging of wrist and finger joints in healthy subjects occasionally shows changes resembling erosions and synovitis as seen in rheumatoid arthritis. Arthritis and rheumatism 50, 1097-1106 (2004).

Baillet, A. et al. Comparison of the efficacy of sonography, magnetic resonance imaging and conventional radiography for the detection of bone erosions in rheumatoid arthritis patients: a systematic review and meta-analysis. Rheumatology (Oxford) 50, $1137-1147$ (2011).

Geusens, P. et al. High-resolution in vivo imaging of bone and joints: a window to microarchitecture. Nat Rev Rheumatol 10, 304-313 (2014).

Dohn, U. M. et al. Detection of bone erosions in rheumatoid arthritis wrist joints with magnetic resonance imaging, computed tomography and radiography. Arthritis Res Ther 10, R25 (2008). Stach, C. M. et al. Periarticular bone structure in rheumatoid arthritis patients and healthy individuals assessed by high-resolution computed tomography. Arthritis and rheumatism 62, 330-339 (2010).

1 Srikhum, W. et al. Quantitative and semiquantitative bone erosion assessment on high-resolution peripheral quantitative computed tomography in rheumatoid arthritis. The Journal of rheumatology 40, 408-416 (2013).

Albrecht, A. et al. The structural basis of MRI bone erosions: an assessment by microCT. Annals of the rheumatic diseases 72, 1351-1357 (2013).

Finzel, S. et al. A detailed comparative study of high-resolution ultrasound and micro-computed tomography for detection of arthritic bone erosions. Arthritis and rheumatism 63, 1231-1236 (2011). Fouque-Aubert, A. et al. Assessment of hand bone loss in rheumatoid arthritis by high-resolution peripheral quantitative CT. Annals of the rheumatic diseases 69, 1671-1676 (2010). inzel, S. et al. Repair of bone erosions in rheumatoid arthritis treated with tumour necrosis factor inhibitors is based on bone apposition at the base of the erosion. Annals of the rheumatic diseases 70, 1587-1593 (2011). lesions in rheumatoid arthritis and psoriatic arthritis. Annals of the rheumatic diseases 70, 122-127 (2011). with rheumatoid arthritis: a cross-sectional HR-pQCT study. Journal of bone and mineral research : the official journal of the American Society for Bone and Mineral Research 29, 1005-1014 (2014).

Aschenberg, S. et al. Catabolic and anabolic periarticular bone changes in patients with rheumatoid
arthritis: a computed tomography study on the role of age, disease duration and bone markers. Arthritis research \& therapy 15, R62 (2013).

Topfer, D., Finzel, S., Museyko, O., Schett, G. \& Engelke, K. Segmentation and quantification of bone erosions in high-resolution peripheral quantitative computed tomography datasets of the metacarpophalangeal joints of patients with rheumatoid arthritis. Rheumatology 53, 65-71 (2014). , Szabo, E. Martin, L, Boyd, S. K. \& Barr, S. G. Quantification of small ioint space width, periarticular bone microstructure and erosions using high-resolution peripheral quantitative computed tomography in rheumatoid arthritis. Clinical and experimental rheumatology 31, 243-250 (2013).

21 Finzel, S. et al. Interleukin-6 receptor blockade induces limited repair of bone

22 Zebaze, R. \& Seeman, E. Cortical bone: a challenging geography. Journal of bone and mineral research : the official journal of the American Society for Bone and Mineral Research 30, 24-29 (2015).

Schett, G. \& Gravallese, E. Bone erosion in rheumatoid arthritis: mechanisms, diagnosis and treatment. Nature reviews. Rheumatology 8, 656-664 (2012).

Burghardt, A. J., Buie, H. R., Laib, A., Majumdar, S. \& Boyd, S. K. Reproducibility of direct quantitative measures of cortical bone microarchitecture of the distal radius and tibia by HR-pQCT. Bone 47, 519-528 (2010).

Pialat, J. B., Burghardt, A. J., Sode, M., Link, T. M. \& Majumdar, S. Visual grading of motion induced image degradation in high resolution peripheral computed tomography: impact of image quality on measures of bone density and micro-architecture. Bone 50, 111 1-1 18 (2012).

Aletaha, D. et al. 2010 Rheumatoid arthritis classification criteria: an American College of Rheumatology/ European League Against Rheumatism collaborative initiative. Arthritis and rheumatism 62, 2569-2581 (2010). 
cortical and trabecular compartments based on a dual threshold technique for in vivo micro-CT bone analysis. Bone 41, 505-515 (2007). Laib, A., Hauselmann, H. J. \& Ruegsegger, P. In vivo high resolution 3D-QCT of the human forearm. Technology and health care : official journal of the European Society for Engineering and Medicine 6, 329 -337 (1998).

Scharmga, A. et al. Visual detection of cortical breaks in hand joints: reliability and validity of highresolution peripheral quantitative CT compared to microCT. BMC musculoskeletal disorders 17, 271 (2016).

Barnabe, C. et al. Definition for Rheumatoid Arthritis Erosions Imaged with High Resolution Peripheral Quantitative Computed Tomography and Interreader Reliability for Detection and Measurement. The Journal of rheumatology 43, 1935-1940 (2016).

31 Landis, J. R. \& Koch, G. G. The measurement of observer agreement for categorical data. Biometrics 33, 159-174 (1977).

32 MacNeil, J. A. \& Boyd, S. K. Accuracy of high-resolution peripheral quantitative computed tomography for measurement of bone quality. Medical engineering \& physics 29, 1096-1 105 (2007). Liu, X. S. et al. High-resolution peripheral quantitative computed tomography can assess microstructural and mechanical properties of human distal tibial bone. Journal of bone and mineral research : the official journal of the American Society for Bone and Mineral Research 25, $746-756$ (2010). 


\section{Supplementary files}

a.
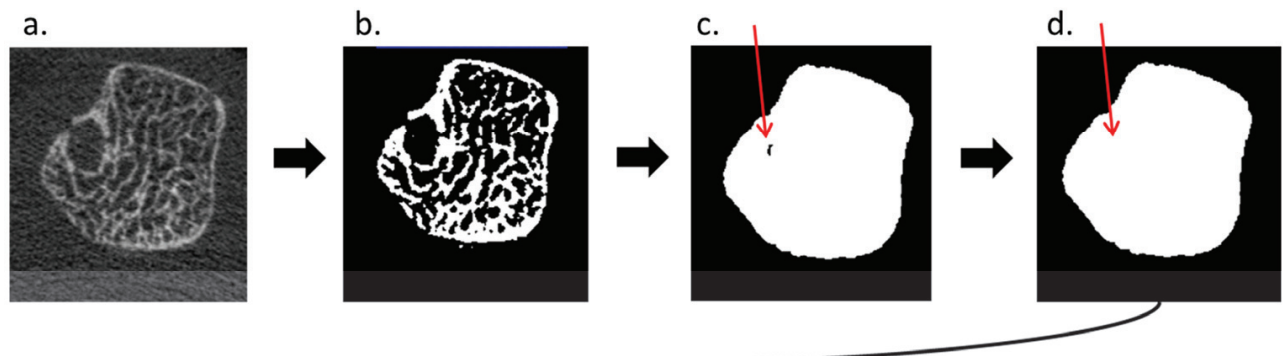

e.

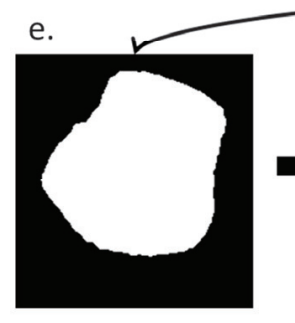

f.

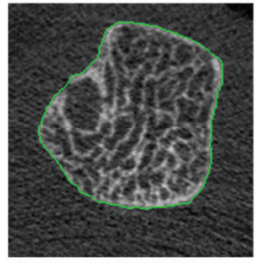

g.

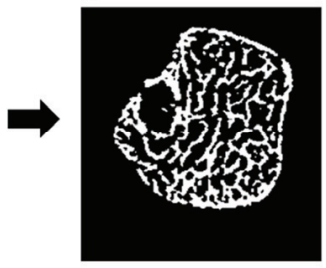

Supplementary figure 7.1 An illustration of the auto-contouring script. An illustration of the autocontouring script of a 2D grayscale image with a cortical interruption. The grayscale image (a) is thresholded for a first structure approximation using Gaussian filtering (sigma $=0.8$, support $=1$ voxel) and a constant threshold of 105 per 1000 of maximum possible voxel value (b). This structure is dilated by 7 voxels (c). After deleting (by ranking) the black voxels inside the dilated structure (red arrow, d), the volume was eroded back to its original size (e). The contour that is obtained is displayed in green in the original grayscale image $(f)$, and used for segmentation of the bone using the standard evaluation protocol with a constant threshold using Laplace-Hamming filtering (g).

Supplementary file 7.1 : MP_InterruptionDetection_246um.com; 1

Code of the algorithm for (semi-)automated detection of interruptions $\geq 0.246 \mathrm{~mm}$.

Supplementary file 7.2 : DATA OP1-OP2-AUTO-Visual_FINAL.sav.

Data of the number of interruptions detected with the algorithm and visually.

\section{Supplementary file 7.3 : AlgorithmVisual_MatchingBreaks.xlsx.}

Data of the number of matching interruptions of the algorithm and visual scoring. Thus, between AUTOOP1, AUTO-OP2, OP1-OP2, and AUTO-Visual, OP1-Visual and OP2-Visual. 


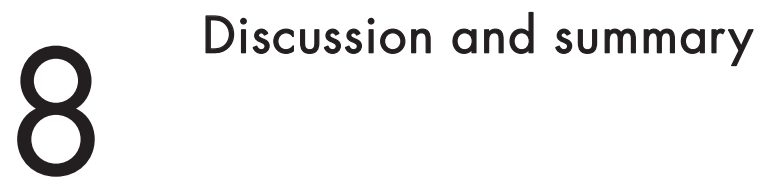




\section{Background}

Rheumatoid arthritis (RA) is a chronic and potentially disabling disease, affecting about one percent of the population'. The disease primarily affects the joints, where structural damage can occur, already at an early stage ${ }^{1,2}$. In RA imaging is essential in the evaluation of structural damage, such as large interruptions of the cortex, i.e. erosions, on conventional radiography (CR). In clinical practice, $C R$ is the gold standard for the assessment of structural damage in the hands and feet of a patient with RA ${ }^{3.7}$. CR is fast and easy to perform, however, disadvantages of the use of CR are that it is not very sensitive for the detection of structural damage at an early stage, nor for the detection of progression of structural damage over time, and that scoring structural damage can be time consuming and may be hindered by projectional superimposition ${ }^{5,8-10}$. More sensitive techniques to detect early structural changes in the joints are computed tomography (CT), ultrasound (US) and magnetic resonance imaging (MRI) $)^{11-14}$. In contrast to $C R$, US and MRI can visualize soft tissue, which enables early detection of inflammatory changes ${ }^{13,15-17}$. Also bone marrow edema (BME) can be visualized using MRI I5,18,19. The (sole) use of US and MRI also has disadvantages such as operator dependency, inability to penetrate the bone (US), limited availability and expensive in use (MRI).

High-Resolution peripheral Quantitative Computed Tomography (HR-pQCT) is a novel imaging technique that allows detailed evaluation of cortical and trabecular structures with an isotropic voxel size of $82 \mu \mathrm{m}$ with less than 3 microSievert of radiation during a standard scan. Originally, HR-pQCT was developed to study bone microarchitecture in metabolic diseases in more detail and to calculate bone strength ${ }^{20.22}$. Recently, the quantification of bone changes, the measurements of size of interruptions of the cortex, and the anatomical sites prone to pathological interruptions in the peri-articular regions of the finger joints, have been studied with HR-pQCT in RA ${ }^{23-27}$. These studies mainly focused on large interruptions of the cortex $(>1.9 \mathrm{~mm})$, considered as RA specific erosions: i.e. an interruption in the cortex accompanied by trabecular bone loss. It was also reported that HR-pQCT enabled detection of very small $(<0.5 \mathrm{~mm})$ cortical interruptions without adjacent trabecular bone $\operatorname{loss}^{24,25}$ and that interruptions of the cortex were also present in healthy individuals ${ }^{24,25}$. The Study grouP for xtrEme Computed Tomography in Rheumatoid Arthritis (SPECTRA) collaboration proposed that some cortical interruptions, e.g. those interruptions having a typical parallel lining, represent physiologic vascular channels (VCs) ${ }^{28}$.

According to the Outcome Measures in Rheumatology (OMERACT) filter, an instrument such as HR-pQCT needs to meet the requirements defined by the filters truth, discrimination and feasibility ${ }^{29,30}$. In this thesis we therefore studied aspects of truth (validity), discrimination (reliability), and feasibility of HR-pQCT with respect to the detection of cortical interruptions in finger joints. Data from subjects from the MOSA- 
Hand cohort, including 41 patients with RA and 38 healthy individuals, and anatomic specimens were used for this purpose.

Summary of the thesis

Previous studies using HR-pQCT in RA were mainly focused on large cortical interruptions and occasionally the existence of small, possible physiological vascular channels was reported, also in healthy individuals ${ }^{24,28}$. Often, other imaging modalities were used in comparison with HR-pQCT imaging 24,25,28. In chapter 2 therefore, we presented an overview of the spectrum of cortical interruptions detected by HR-pQCT imaging in rheumatology. HR-pQCT, MRI and CR imaging data from finger joints of patients with RA and healthy subjects were used and the heterogeneity of cortical interruptions using HR-pQCT imaging in metacarpophalangeal (MCP) joints was shown. We also confirmed that the presence and extent of bone damage observed with HR$\mathrm{pQCT}$ is more pronounced and complex than observed with $\mathrm{CR}^{23,24,31-33}$.

At the start of this thesis, there was a knowledge gap regarding the performance of HRPQCT in the detection of small cortical interruptions. In chapter 3, we therefore investigated the truth and discrimination of visual detection of cortical interruptions in MCP and proximal interphalangeal (PIP) joints when using HR-pQCT with $\mu C T$ as gold standard. Nineteen joints of female anatomic specimens (ten MCP and nine PIP joints) were analyzed for the presence of cortical interruptions on HR-pQCT and $\mu C T$. We studied the intra- and inter-reader reliability and determined the sensitivity, specificity, positive predictive value (PPV) and negative predictive value (NPV). Using either imaging modality, cortical interruptions were found in all joints (129 on HR-pQCT and 149 on $\mu \mathrm{CT}$ ). Intra-reader reliability for the presence (kappa, $\mathrm{k}$ ) and total number (intraclass correlation coefficient, ICC) of cortical interruptions was moderate to substantial (range $0.52-0.61$ ) for HR-pQCT and substantial (range $0.71-0.75$ ) for $\mu C T$. Inter-reader reliability for the presence (K) and total number (ICC) of cortical interruptions was fair to moderate (range $0.37-0.55$ ) for HR-pQCT and moderate (range $0.45-0.54)$ for $\mu C T$. HR-pQCT appeared highly sensitive $(81.6 \%)$ to detect cortical interruptions with $\mu \mathrm{CT}$ as gold standard. PPV and NPV were $81.6 \%$ and $64.0 \%$, respectively. In conclusion, cortical interruptions were commonly detected in finger joints on both HR-pQCT and $\mu \mathrm{CT}$ with fair to substantial reliability and high sensitivity.

To further clarify the nature of the cortical interruptions we compared the image findings on HR-pQCT with histological analyses, a type of study that had not been performed before. In chapter 4, we therefore first studied whether cortical interruptions detected in peri-articular regions in MCP joints and classified as VCs on HR-pQCT using the SPECTRA definition, were indeed VCs on histology. Subsequently, we evaluated the image characteristics of histologically identified VCs to matched single (axial plane) and multiplane slices of HR-pQCT images. Four peri-articular regions in three MCP joints of anatomic specimens were selected because they contained high numbers of cortical interruptions, and these were histologically examined. First, of seven initially suggested 
VCs according to the SPECTRA definition on HR-pQCT, i.e. an interruption of the cortex with a parallel structure present on two consecutive slices in two planes (2x2 slices), only one appeared to be a true VC on histology. Second, we found 52 (small) VCs on histological sections, which had a heterogeneous image pattern on HR-pQCT. On matched single slice HR-pQCT images, 25 of the 52 VCs presented as full cortical interruptions that penetrated the cortex in a straight or oblique direction. Fourteen of the 52 VCs had no complete interruption of the cortex but presented as periosteal excavations. On multiplane HR-pQCT images, 11 VCs appeared on at least $2 \times 2$ slices and 36 VCs in less than $2 \times 2$ slices. Not all histologically identified VCs could be identified on single and multiplane HR-pQCT images (13 and 5 VCs, respectively). The width of histologic VCs ranged between 0.049 and $0.790 \mathrm{~mm}$. Importantly, 6 of the 52 VCs were smaller than the voxel size of HR-pQCT $(82 \mu \mathrm{m})$. We concluded that the heterogeneous presentation of the histologically identified VCs, the small size of many VCs and the voxel size limit the detection of VCs on HR-pQCT images. Furthermore, the preliminary SPECTRA definition appeared to be insufficient to capture most VCs using HR-pQCT.

Small cortical interruptions, or vascular channels, have not been incorporated in scoring algorithms for HR-pQCT images before. Because of the ability of HR-pQCT to also sensitively detect small cortical interruptions (chapter 3), we developed a visual scoring method incorporating all cortical interruptions, described in chapter 5, and tested its truth and feasibility in a sample of 30 subjects of the MOSA-Hand cohort 110 healthy subjects and 20 patients with RA). For development of the scoring method, we integrated results from in-depth discussions with experts, consensus meetings, multiple reading experiments ${ }^{34,35}$, published studies on grading of motion artefacts ${ }^{36}$ and assessment of finger joints using HR-pQCT images ${ }^{24}$. The experts consisted of a panel of rheumatologists, radiologists and engineers specialized in image analyses. The visual scoring method that was developed included also scoring of small cortical interruptions, present on one slice in addition to two consecutive slices in another plane (1x2 slices), with or without adjacent trabecular distortion. When applying this method, a total 252 cortical interruptions could be identified (range diameter interruptions $0.09 \mathrm{~mm}-7.40$ $\mathrm{mm}$ ), both in healthy subjects (mean (SD) 1.9 (1.7)) and in patients with RA (mean 2.9 (2.4)). Adjacent trabecular distortion was observed only in patients with RA, in $21 \%$ of the cortical interruptions. In patients with RA, compared with healthy subjects, significantly more cortical interruptions were observed in the ulnar (mean $\pm S D, 0.5 \pm 0.9$ versus $0.6 \pm 0.9)$ and radial quadrants $(0.5 \pm 0.6$ versus $0.1 \pm 0.3, p<0.05)$. Cortical interruptions were also frequently seen in the palmar quadrants, but equally in patients with RA and healthy subjects. Intra-reader reliability for the presence $(K)$ and total number (ICC) of cortical interruptions was moderate to almost perfect per joint 10.52 0.88 ) and substantial per quadrant $(0.63-0.69)$. Inter-reader reliability for the presence and total number of cortical interruptions was fair to moderate per joint 10.37 - 0.48), and moderate $(0.53-0.56)$ per quadrant. The mean time to score one joint was 9.2 (SD 4.9) min. In conclusion, this scoring method enables visual detection of 
small cortical interruptions when using HR-pQCT imaging of finger joints. Although reading was time-consuming, this approach is promising for use in clinical studies.

To further validate the use of HR-pQCT, we applied the visual scoring method in finger joints of HR-pQCT images of the MOSA-Hand cohort. In chapter 6, we were interested to study on the one hand the relationship between structural features on MRI and radiography with presence or number of cortical interruptions in finger joints on HRpQCT in patients with RA and healthy subjects. On the other hand, we wanted to evaluate whether several other risk features for structural damage, such as bone marrow edema (BME) on MRI, anti-citrullinated protein antibody (ACPA) and rheumatoid factor $\lg M(R F)$ seropositivity, hand dominance and disease duration are also associated with the number of cortical interruptions on HR-pQCT. The finger joints of 38 healthy subjects and 39 patients with RA were examined through radiographs, MRI and HR-pQCT. Radiographic damage was assessed using the Sharp/van der Heijde method. Cortical interruptions, BME and synovitis were scored on MRI. Cortical interruptions were scored on HR-pQCT. Cortical interruptions, with or without adjacent trabecular distortion were seen in both healthy subjects and patients with RA on HR-pQCT, but more frequently in patients with RA. A significant association was observed between structural damage on either MRI or radiographs with the presence of cortical interruptions on HR-pQCT (respectively OR 12.4, 95\% Cl 7.5-21.4, $\mathrm{p}<0.01$ and $\mathrm{OR} 4.8,95 \% \mathrm{Cl} 1.9-11.7$, $p<0.01)$. The presence of either BME or synovitis, was associated with more cortical interruptions on HR-pQCT (B 0.47, 95\% Cl 0.4-0.6, p<0.01 and $\mathrm{B} 1.9,95 \% \mathrm{Cl} 0.6$ $3.1, p<0.011$. In patients with RA, ACPA and/or RF seropositivity, hand dominance and disease duration were not associated with more cortical interruptions on HR-QCT. The accomplishment of the visual scoring method indicated the need for a more feasible (semi-) automated algorithm. While there was one published paper reporting accurate detection of large cortical interruptions, with an algorithm that still relied on operator input ${ }^{27}$, a fully-automated algorithm to detect cortical interruptions was not available yet. In chapter 7, we therefore developed a fully-automated algorithm to detect cortical interruptions on HR-pQCT and tested it using HR-pQCT images of MCP joints from patients with RA and healthy subjects from the MOSA-Hand cohort. Also, the additional value of manual correction of automatically generated periosteal contours was investigated (semi-automated approach). The median number of cortical interruptions per joint detected with the algorithms was 14 (range 2 - 59) and did not differ significantly between the semi- and fully-automated algorithms $(p=0.37)$. Almost perfect reliability (ICC $0.95-0.97$ ) to detect cortical interruptions was observed for both the fully- and semi-automated algorithm procedures. Compared to visual detection of cortical interruptions, considered the gold standard in this study, reliability of the fullyand semi-automated algorithms was fair to moderate (ICC $0.38-0.45$ ). The majority of the interruptions detected visually were also detected by the fully- and semiautomated algorithms $(70-76 \%)$. In this study, the semi-automated procedure appeared of little additional value however, the semi-automated procedure is still advised in case of large cortical interruptions and considerable motion artefacts. In conclusion, the 
application of the fully automated algorithm is promising for a sensitive and objective assessment of cortical interruptions in fingers joints by HR-pQCT.

\section{Discussion of main findings}

'Truth' of HR-pQCT

Questions such as "Is the measure truthful, does it measure what is intended?" and "Is the result unbiased and relevant?" reflects the OMERACT filter 'truth'29. At the start of this thesis, evidence was lacking from gold standards ( $\mu$ CT and histology) for the correct interpretation of HR-pQCT images of the peri-articular regions in finger joints. In this thesis, the first steps were taken to answer the questions of the filter "truth". In chapter 3 , we showed that HR-pQCT is a sensitive (81.6\%) imaging technique to detect cortical interruptions using $\mu \mathrm{CT}$ as gold standard, with a PPV of also $81.6 \%$. In addition, cortical interruptions that were present in at least $2 \times 2$ slices were frequently seen $(n=$ 149) in MCP and PIP joints of anatomic specimens although the nature of these cortical interruptions, i.e. pathological erosions or physiological VCs, remained unknown in our study. It has been hypothesized that bone damage in RA might start in VCs ${ }^{37-39}$. In chapter 4, we therefore performed further research that focused on histological analyses to clarify the nature of the cortical interruptions suspected for a VC on HRpQCT. Only one out of five evaluable VCs identified on HR-pQCT according to the SPECTRA definition appeared a true VC on histology. Subsequently, we showed that HR-pQCT was able to show 39 out of 52 histologically identified VCs with a heterogeneous pattern on single, axial plane HR-pQCT slices and 47 out of 52 VCs on multiplane HR-pQCT images. The current SPECTRA definition of a VC using HR-pQCT imaging appeared insufficient to correctly identify VCs due to heterogeneity in their pattern on HR-pQCT images. A challenge lies in the formation of a new definition. VCs were small in size $(0.049-0.790 \mathrm{~mm})$ and some were also smaller than $2 \times 2$ slices. Preferably, a new definition incorporates cortical interruptions smaller than $2 \times 2$. However, not all histologically identified VCs could be seen on single and multiplane HR-pQCT images (35\% $(n=13)$ and 10\% $(n=5)$ VCs, respectively). Moreover, our results showed that 6 out of 52 VCs were smaller than the voxel size of HR-pQCT (82 $\mu \mathrm{m})$. Thus, the heterogeneous appearance and small size of the VCs limit the identification as such on HR-pQCT.

Truth of HR-pQCT was further explored on content validity in chapter 6, where the visual scoring method for cortical interruptions on HR-pQCT was applied to images of finger joints in healthy subjects and patients with RA. We showed that cortical interruptions were seen in healthy subjects and patients with RA, with significantly more cortical interruptions in the latter group. We observed a significant association of structural damage on MRI or radiographs with the presence of cortical interruptions on HR-pQCT. When BME or synovitis was present, significantly more cortical interruptions were found on HR-pQCT. Our results were in line with previous studies, which showed that the presence of $B M E$ on $M R I$ is associated with cortical interruptions and progression of structural bone damage on radiographs ${ }^{40-42}$. Furthermore, the 
association demonstrated between BME on MRI and presence of cortical interruptions on HR-pQCT in healthy subjects has also been previously described ${ }^{43}$. In patients with RA, we were however unable to demonstrate an association between ACPA and/or RF seropositivity and number of cortical interruptions on HR-pQCT in patients with RA. This has been demonstrated before for ACPA and/or RF seropositivity and radiographic damage ${ }^{44}$. For this study, we used the visual scoring method for HR-pQCT, which takes into account all cortical interruptions. No distinction was made between physiological vascular channels or pathological erosions. We assessed adjacent trabecular distortion, which could be suggestive for an erosion. However, this has not yet been confirmed by histology. Future research, preferably including also histologically sectioning, should therefore further explore the nature of cortical interruptions.

\section{'Discrimination' of HR-pQCT}

A question such as "Does the measure discriminate between situations of interest?" reflects the OMERACT filter 'discrimination'29. According to OMERACT, situations can be states at one or at different times and capture issues of reliability and sensitivity to change ${ }^{29}$. Several studies developed scoring instruments for HR-pQCT images, focussing on large and established erosions. Stach et al. developed a semi-quantitative scoring method for erosions that were graded based on severity. Intra- and inter-reader reliability showed good results $\left(k=0.82\right.$ and $k=0.75$ respectively) ${ }^{24}$. Also Srikhum et al. developed a method to measure and grade erosions quantitatively and semiquantitatively. Intra- and inter-reader reliability results were almost perfect (ICC 0.99 and ICC 0.89 respectively $)^{25}$. In this thesis, we took additional steps to answer the questions of the filter "discrimination". In chapters 3 and 5 intra- and inter-reader reliability were assessed for the detection of cortical interruptions on HR-pQCT using $\mu C T$ as gold standard in anatomic specimens, and for the detection of cortical interruptions using a visual scoring method in patients with RA and healthy subjects. Intra-reader reliability in both studies was moderate to substantial for the presence of a cortical interruption (lowest $\mathrm{k} 0.52(95 \% \mathrm{Cl} 0.48 ; 0.56)$ - highest $\mathrm{k} 0.63(95 \% \mathrm{Cl}$ $0.56 ; 0.691)$ and substantial to almost perfect for the total number of cortical interruptions (lowest ICC 0.61 (95\% Cl 0.49;0.70) - highest ICC $0.88195 \% \mathrm{Cl}$ $0.83 ; 0.92))$. Inter-reader reliability in both studies was fair to moderate for the presence of cortical interruptions (lowest ( $\mathrm{k}) 0.37(95 \% \mathrm{Cl} 0.33 ; 0.41)$ - highest $\mathrm{k} 0.53(95 \% \mathrm{Cl}$ $0.52 ; 0.54)$ ) and was moderate for the total number of cortical interruptions (lowest ICC $0.48(95 \% \mathrm{Cl} 0.20 ; 0.67)$ - highest ICC 0.56 (95\% Cl 0.49;0.62)). Reliability of other imaging techniques such as conventional radiographs also ranged between moderate and almost perfect (ICC 0.47 - 0.99), despite being scored by experienced readers ${ }^{45}$. The visual interpretation of HR-pQCT images by multiple readers remains a complex task and should be taken into consideration when developing a scoring method or when applying the visual scoring method, including education and training. In both studies described in chapter 3 and chapter 5, large discrepancies (differences of $>3$ cortical interruptions) between readers were found and further evaluated. First, it was 
observed that the smaller the interruption, the less agreement. $\mu C T$ images provided higher detail, and particularly very small cortical interruptions were not always picked up by the readers (chapter 3). Second, low mineralization and/or thin cortices made interruptions difficult to distinguish from the background noise. Third, larger interruptions showed more agreement, but sometimes, in the case of a large interruption identified by one reader, multiple smaller interruptions within such interruption were counted as individual interruptions by another reader. Reliability can be improved by focusing on large interruptions only, accompanied by loss of trabecular structure. However, the strength of HR-pQCT imaging is its ability to identify also small cortical interruptions. Therefore, there was need for a reliable method to score (small) cortical interruptions on HR-pQCT images. In chapter 7 we showed that a fully automated algorithm for the detection of cortical interruptions by HR-pQCT had promising results with an almost perfect reliability (ICC $0.96(95 \% \mathrm{Cl} 0.87 ; 0.99)$ ). Compared to previous studies, the algorithm also detected smaller interruptions than visual algorithms $1<1.13 \mathrm{~mm}$ compared to $>2 \mathrm{~mm})^{46,47}$. These results will make the algorithm interesting for application in clinical trials, but the ability of the algorithm to detect progression of bone damage over time still needs to be tested.

\section{'Feasibility' of HR-pQCT}

A question such as "Can the measure be applied easily, given constraints of time, money, and interpretability?" reflects the OMERACT filter 'feasibility'29. At the start of this thesis no studies on the feasibility of HR-pQCT use had been performed. Based on our experience, HR-pQCT has a scan time of approximately 15 minutes, when using 330 and 220 slices for the second and third MCP and PIP joints of one hand respectively. Compared to MRI, which takes up to one hour for both hands, it is less time consuming, but not compared with CR of the hands, which takes no more than a few seconds. Furthermore, studies on the time to score HR-pQCT images were scarce. One previous study reported a scoring time for MCP joints per patient and found a median of 2 minutes (range $1.20-5.30$ minutes) ${ }^{25}$. In chapter 5 , we studied the feasibility of a newly developed visual scoring method to score the presence of cortical interruptions in MCP and PIP joints on HR-pQCT images. Using the visual scoring method, reading one joint was on average 9.2 minutes (range 1 - 38 minutes). We believe that in clinical practise it is therefore not feasible to apply this visual scoring method. The results of the visual scoring method indicated the need for a feasible (semi-) automated algorithm. In chapter 7 the proposed automated algorithm showed promising results however feasibility in terms of time to execute the algorithm was not tested.

\section{Limitations}

The studies described in this thesis have several limitations.

First, in chapters 3 and 4 we used a sample of anatomic specimens of elderly women, preserved in formalin, with unknown medical background and with low bone 
mineralization (average volumetric bone mineral density $219.7 \mathrm{mgHA} / \mathrm{cm}^{3}$ compared to $>300 \mathrm{mgHA} / \mathrm{cm}^{3}$ in healthy subjects ${ }^{23,48}$. The condition and visual observations on HR-pQCT imaging might have been affected by the relatively old age and lower mineralization of the specimens. An earlier study showed no significant difference in bone mineral density due to formalin fixation between two groups of fixated and nonfixated specimens ${ }^{49}$. Therefore, we believe that the use of formalin likely did not affect the quality of the specimens. The unknown medical history of the anatomic specimens, however, is a limitation that should be taken into account: extrapolation of the study findings to young, healthy subjects and to patients with RA should be done with caution.

Second, partial volume effects (PVE) are inherent to HR-pQCT imaging. Due to a reduced cortical signal on $\mathrm{HR}-\mathrm{pQCT}$, thin cortices can falsely be identified as cortical interruptions. We showed that HR-pQCT is a clinically feasible technique with high sensitivity $(81.6 \%)$ using $\mu \mathrm{CT}$ as gold standard in chapter 3 . Specificity in this study however was lower $(64 \%)$, probably due to PVEs.

Third, although we demonstrated an association between structural damage, and inflammatory markers with presence and number of cortical interruptions on HR-pQCT, we could not demonstrate an association between ACPA and/or RF seropositivity and number of cortical interruptions on HR-pQCT in patients with RA. This is most likely due to the lack of statistical power due to a low number of patients with RA in the ACPA and RF negative group ( $n=9)$. Also, all patients with RA received treatment, of which more than $50 \%$ using a biological. Using HR-pQCT, it has been reported that large cortical interruptions detected in patients with RA may repair after treatment with tumor necrosis factor inhibitors ${ }^{50}$.

Fourth, motion artefacts are a common finding in HR-pQCT imaging ${ }^{36}$. In chapter 5, we found that less than $8 \%$ of the HR-pQCT images were considered insufficient for further analyses by the visual scoring method due to motion artefact grade $>3$, as described by Pialat $^{36}$. At baseline HR-pQCT scanning, in only four cases out of in total 232 scans, a scan was redone when a motion grade $>3$ was observed in the interim HR-pQCT slice analysis. During the process of scanning, an interim slice is shown after each 110 slices. Although less than $8 \%$ of the HR-pQCT images were considered insufficient, the interim HR-pQCT images were not sufficient to allow good evaluation of motion artefacts in HR-pQCT images in all cases. Normally, in case of motion artefacts on the interim HR-pQCT images, the scan is aborted and a new scan is made. In order to prevent rescanning and unnecessary radiation exposure, it is important that operators provide correct instructions to the subjects before scanning and stress that it is essential not to move the body.

\section{Future perspectives}

$C R$ remains the gold standard to evaluate structural damage in finger joints in patients with RA in clinical practice. HR-pQCT however, has proven to be a reliable and more sensitive imaging method to detect bone changes and smaller cortical interruptions 
compared to CR. This thesis took additional steps in the validation of HR-pQCT scanning of peri-articular regions in finger joints, including studies using gold standards such as $\mu C T$ and histology. Also, a visual and an automated scoring method were developed and tested. Future studies can focus on the role of VCs in the development of erosions. If specific VCs involved in erosion development can be identified by HR$p Q C T$, early screening for bone abnormalities at erosion prone sites in patients with RA could be considered. In this thesis, we have already found a higher number of cortical interruptions in patients with RA at the radial and ulnar sides, the main location of entheses in finger joints.

Future studies could use the second generation HR-pQCT scanner. This scanner has a higher resolution and a smaller voxel size $(61 \mu \mathrm{m})$. Therefore even smaller cortical interruptions can be detected and hence provide more details on VCs in finger joints.

We applied the visual scoring method to HR-pQCT images of finger joints of subjects of the MOSA-Hand cohort in a cross-sectional design. For the first time we evaluated the relationship between structural features on MRI and radiography, inflammatory features on MRI, and other risk factors with presence or number of cortical interruptions on HRpQCT using baseline data. Next, possible associations should be investigated using longitudinal data.

Finally, it would be interesting to use HR-pQCT in clinical trials to evaluate and monitor the effects of therapy. Considering the time consuming application of the visual method, the automated algorithm would be preferred. However, the proposed automated algorithm which already showed promising results, first needs to be further validated, preferably in longitudinal studies.

In addition, given the prospects of high resolution imaging in finger joints, this field of research may be expanded to other bone affecting rheumatic diseases, such as psoriatic arthritis and gout.

\section{Overall conclusion}

This thesis showed that HR-pQCT is a reliable and sensitive tool to identify the heterogeneous spectrum of cortical interruptions, i.e. physiological VCs and pathological erosions, in patients with RA, healthy subjects and anatomic specimens. However, the definition used for a cortical interruption representing a VC, as proposed by the SPECTRA collaboration, is insufficient. In addition, the detection of the (small) VCs is difficult because of the heterogeneous appearance on HR-pQCT images and is still limited by the voxel size of HR-pQCT. A visual scoring method for detecting cortical interruptions on HR-pQCT was developed, with a fair to moderate inter-reader reliability, but poor feasibility for application in clinical practice. The fully automated algorithm for detection of cortical interruptions on HR-pQCT, however, showed promising results and could aid in a feasible detection of small cortical interruptions. Associations between structural damage on MRI and radiographs, and inflammatory features on MRI with the presence and number of cortical interruptions on HR-pQCT were observed using data of our MOSA-Hand cohort. Altogether, this thesis represents 
a solid basis for further application and research using HR-pQCT for imaging of periarticular bone of MCP and PIP joints in patients with RA. 


\section{References}

1 Paleolog, E. M. The vasculature in rheumatoid arthritis: cause or consequence? Int J Exp Pathol 90, 249 261 (2009).

2 van der Heijde, D. M. Joint erosions and patients with early rheumatoid arthritis. Br J Rheumato/ 34 Suppl 2, 74-78 (1995).

3 Boini, S. \& Guillemin, F. Radiographic scoring methods as outcome measures in rheumatoid arthritis: properties and advantages. Ann Rheum Dis 60, 817-827 (2001).

Lillegraven, S., van der Heijde, D., Uhlig, T., Kvien, T. K. \& Haavardsholm, E. A. What is the clinical relevance of erosions and joint space narrowing in RA? Nat Rev Rheumato/ 8, 117-120 (2012).

Ravindran, V. \& Rachapalli, S. An overview of commonly used radiographic scoring methods in rheumatoid arthritis clinical trials. Clin Rheumato/ 30, 1-6 (2011).

Sommer, O. J. et al. Rheumatoid arthritis: a practical guide to state-of-the-art imaging, image interpretation, and clinical implications. Radiographics 25, 381-398 (2005).

van der Heijde, D., Dankert, T., Nieman, F., Rau, R. \& Boers, M. Reliability and sensitivity to change of a simplification of the Sharp/van der Heijde radiological assessment in rheumatoid arthritis. Rheumatology (Oxford) 38, 941-947 (1999).

Mottonen, T. T. Prediction of erosiveness and rate of development of new erosions in early rheumatoid arthritis. Ann Rheum Dis 47, 648-653 (1988).

Nissila, M. et al. Prognosis of inflammatory joint diseases. A three-year follow-up study. Scand J Rheumatol 12, 33-38 (1983).

van der Heijde, D. M. et al. Biannual radiographic assessments of hands and feet in a three-year prospective followup of patients with early rheumatoid arthritis. Arthritis Rheum 35, 26-34 (1992).

11 Ostergaard, M., Pedersen, S. J. \& Dohn, U. M. Imaging in rheumatoid arthritis-status and recent advances for magnetic resonance imaging, ultrasonography, computed tomography and conventional radiography. Best Pract Res Clin Rheumatol 22, 1019-1044 (2008).

Baillet, A et al. Comparison of the efficacy of sonography, magnetic resonance imaging and conventional radiography for the detection of bone erosions in rheumatoid arthritis patients: a systematic review and meta-analysis. Rheumatology (Oxford) 50, $1137-1147$ (2011).

Dohn, U. M. et al. Are bone erosions detected by magnetic resonance imaging and ultrasonography true erosions? A comparison with computed tomography in rheumatoid arthritis metacarpophalangeal joints. Arthritis Res Ther 8, R 110 (2006).

Perry, D. et al. Detection of erosions in the rheumatoid hand; a comparative study of multidetector computerized tomography versus magnetic resonance scanning. J Rheumato/ 32, 256-267 (2005).

Tan, A. L. et al. Role of metacarpophalangeal joint anatomic factors in the distribution of synovitis and bone erosion in early rheumatoid arthritis. Arthritis Rheum 48, 1214-1222 (2003). volumes in rheumatoid arthritis and osteoarthritis: comparison with the macroscopic and microscopic appearance of the synovium. Arthritis Rheum 40, 1856-1867 (1997).

Ostendorf, B. et al. Magnetic resonance imaging and miniarthroscopy of metacarpophalangeal joints: sensitive detection of morphologic changes in rheumatoid arthritis. Arthritis Rheum 44, 2492-2502 (2001). McGonagle, D. et al. The relationship between synovitis and bone changes in early untreated rheumatoid arthritis: a controlled magnetic resonance imaging study. Arthritis Rheum 42, 1706-1711 (1999). Krabben, A. et al. MRI-detected subclinical joint inflammation is associated with radiographic progression. Ann Rheum Dis 73, 2034-2037 (2014).

20 Boutroy, S., Bouxsein, M. L., Munoz, F. \& Delmas, P. D. In vivo assessment of trabecular bone microarchitecture by high-resolution peripheral quantitative computed tomography. J Clin Endocrinol Metab 90, 6508-6515 (2005).

Laib, A., Hauselmann, H. J. \& Ruegsegger, P. In vivo high resolution 3D-QCT of the human forearm. Technol Health Care 6, 329-337 (1998).

MacNeil, J. A. \& Boyd, S. K. Accuracy of high-resolution peripheral quantitative computed tomography for measurement of bone quality. Med Eng Phys 29, 1096-1 105 (2007).

23 Fouque-Aubert, A. et al. Assessment of hand bone loss in rheumatoid arthritis by high-resolution peripheral quantitative CT. Ann Rheum Dis 69, 1671-1676 (2010).

24 Stach, C. M. et al. Periarticular bone structure in rheumatoid arthritis patients and healthy individuals assessed by high-resolution computed tomography. Arthritis Rheum 62, 330-339 (2010).

Srikhum, W. et al. Quantitative and semiquantitative bone erosion assessment on high-resolution peripheral quantitative computed tomography in rheumatoid arthritis. J Rheumatol 40, 408-416 (2013).

Barnabe, C. \& Feehan, L. High-resolution peripheral quantitative computed tomography imaging protocol for metacarpophalangeal joints in inflammatory arthritis: the SPECTRA collaboration. J Rheumatol 39, 1494-1495 (2012).

Topfer, D., Finzel, S., Museyko, O., Schett, G. \& Engelke, K. Segmentation and quantification of bone erosions in high-resolution peripheral quantitative computed tomography datasets of the metacarpophalangeal joints of patients with rheumatoid arthritis. Rheumatology (Oxford) 53, 65-71 (2014).

Finzel, S. et al. A detailed comparative study of high-resolution ultrasound and micro-computed tomography for detection of arthritic bone erosions. Arthritis Rheum 63, 1231-1236 (2011). 
Boers, M., Kirwan, J. R. \& Tugwell, P. The OMERACT Handbook. (2015).

Boers, M., Brooks, P., Strand, C. V. \& Tugwell, P. The OMERACT filter for Outcome Measures in Rheumatology. J Rheumatol 25, 198-199 (1998).

Barnabe, C., Szabo, E., Martin, L., Boyd, S. K. \& Barr, S. G. Quantification of small joint space width, periarticular bone microstructure and erosions using high-resolution peripheral quantitative computed tomography in rheumatoid arthritis. Clin Exp Rheumatol 31, 243-250 (2013).

Hecht, C. et al. Additive effect of anti-citrullinated protein antibodies and rheumatoid factor on bone erosions in patients with RA. Ann Rheum Dis (2014).

Lee, C. H. et al. Correlation of structural abnormalities of the wrist and metacarpophalangeal joints evaluated by high-resolution peripheral quantitative computed tomography, 3 Tesla magnetic resonance imaging and conventional radiographs in rheumatoid arthritis. Int J Rheum Dis (2014).

Scharmga, A. et al. Visual detection of cortical breaks in hand joints: reliability and validity of highresolution peripheral quantitative CT compared to microCT. BMC Musculoskelet Disord 17, 271 (2016).

Scharmga A, K. K., Peters M, van Tubergen A, van den Bergh J, van Rietbergen B, Weijers R, Loeffen D, Hauge EM, Geusens P. in Arthritis Rheumatol. 2016; 68 (suppl 10).

Pialat, J. B., Burghardt, A. J., Sode, M., Link, T. M. \& Majumdar, S. Visual grading of motion induced image degradation in high resolution peripheral computed tomography: impact of image quality on measures of bone density and micro-architecture. Bone 50, 111 -11 18 (2012).

Schett, G. \& Gravallese, E. Bone erosion in rheumatoid arthritis: mechanisms, diagnosis and treatment. Nat Rev Rheumatol 8, 656-664 (2012).

Binks, D. A. et al. Role of vascular channels as a novel mechanism for subchondral bone damage at cruciate ligament entheses in osteoarthritis and inflammatory arthritis. Ann Rheum Dis 74, 196-203 (2015). Schett, G. et al. Analysis of the kinetics of osteoclastogenesis in arthritic rats. Arthritis Rheum 52, $3192-$ 3201 (2005).

Gandjbakhch, F., Foltz, V., Mallet, A., Bourgeois, P. \& Fautrel, B. Bone marrow oedema predicts structural progression in a 1-year follow-up of 85 patients with RA in remission or with low disease activity with lowfield MRI. Ann Rheum Dis 70, $2159-2162$ (2011).

Hetland, M. L. et al. MRI bone oedema is the strongest predictor of subsequent radiographic progression in early rheumatoid arthritis. Results from a 2 -year randomised controlled trial (CIMESTRA). Ann Rheum Dis 68, 384-390 (2009).

Haavardsholm, E. A., Boyesen, P., Ostergaard, M., Schildvold, A. \& Kvien, T. K. Magnetic resonance imaging findings in 84 patients with early rheumatoid arthritis: bone marrow oedema predicts erosive progression. Ann Rheum Dis 67, 794-800 (2008).

Mangnus, L., van Steenbergen, H. W., Reijnierse, M. \& van der Helm-van Mil, A. H. Magnetic Resonance Imaging-Detected Features of Inflammation and Erosions in Symptom-Free Persons From the General Population. Arthritis Rheumatol 68, 2593-2602 (2016).

Courvoisier, N. et al. Prognostic factors of 10 -year radiographic outcome in early rheumatoid arthritis: a prospective study. Arthritis Res Ther 10, R106 (2008).

Sharp, J. T. et al. Variability of precision in scoring radiographic abnormalities in rheumatoid arthritis by experienced readers. J Rheumato/ 31, 1062-1072 (2004).

Finzel, S. et al. Repair of bone erosions in rheumatoid arthritis treated with fumour necrosis factor inhibitors is based on bone apposition at the base of the erosion. Annals of the rheumatic diseases 70, 1587-1593 (2011). arthritis: a micro CT study. Annals of the rheumatic diseases 72, 396-400 (2013).

Feehan, L., Buie, H., Li, L. \& McKay, H. A customized protocol to assess bone quality in the metacarpal head, metacarpal shaft and distal radius: a high resolution peripheral quantitative computed tomography precision study. BMC Musculoskelet Disord 14, 367 (2013).

Edmondston, S. J., Singer, K. P., Day, R. E., Breidahl, P. D. \& Price, R. I. Formalin fixation effects on vertebral bone density and failure mechanics: an in-vitro study of human and sheep vertebrae. Clin Biomech (Bristol, Avon) 9, 175-179 (1994).

Finzel, S. et al. Repair of bone erosions in rheumatoid arthritis treated with tumour necrosis factor inhibitors is based on bone apposition at the base of the erosion. Ann Rheum Dis 70, 1587-1593 (2011). 


\title{
Addendum
}

\author{
Valorisation
}

Nederlandse samenvatting

Dankwoord

Curriculum Vitae 



\section{Valorisation}

This valorisation chapter describes how the outcomes of this thesis contribute to society and specifically for research and industry. We think that the results described in this thesis are of relevance to future research using High-Resolution peripheral Quantitative Computed Tomography (HR-pQCT), especially on the role of vascular channels in bone damage in rheumatoid arthritis (RA) and in other bone affecting diseases.

First, we thank our participants for their voluntary participation in our studies. They volunteered to image their finger joints, but the results described in this thesis were not common knowledge to most of our participants. Because the gathered data and results, as described in this thesis, were not common knowledge to most of our participants we kept them informed on the progress and scientific outcomes we sent annual newsletters in layman language. This way, the subjects became more aware of the value of their participation and this simple form of information sharing was received with great gratitude. It is therefore recommendable to other research groups to do the same.

\section{Background}

RA is a chronic inflammatory autoimmune disease affecting approximately one percent of the population worldwidel. Patients with RA often suffer from inflammation of the joints. The subsequent bone damage caused by inflammation might lead to progressive deformity and disability of, for example, the finger joints. When damage in the finger joints is present early in the RA disease course, this forms a risk factor for poor longterm disease outcome $e^{2,3}$. In clinical practice of a rheumatologist, conventional radiography is still used as gold standard to assess damage and progression of damage in finger joints of patients with RA.

In the management of patients with RA, other and more sensitive imaging techniques are suggested for monitoring disease activity and progression of damage ${ }^{4}$. For example, compared to conventional radiography, HR-pQCT is proven to be a more sensitive imaging technique for detection of cortical interruptions ${ }^{5}$. In this thesis, we studied especially the presence of cortical interruptions in finger joints in patients with RA and healthy subjects using HR-pQCT. It was previously suggested that cortical interruptions on HR-pQCT can represent either physiological vascular channels or pathological erosions, which are considered specific for RA ${ }^{6}$. Pathological interruptions represent damage that has already occurred; physiological vascular channels on the other hand, may play a role in the development of a large interruption. Vascular channels allow direct communication between the bone marrow and the surrounding joint structures ${ }^{Z}$. In one of the chapters of this thesis, we studied the nature of cortical interruptions seen on HR-pQCT, using histology as the gold standard. We studied whether cortical interruptions detected in metacarpophalangeal joints and classified as vascular channels on HR-pQCT were indeed vascular channels on histology. Also, we evaluated the image characteristics of histologically identified vascular channels on 
matched HR-pQCT images. Our results showed that vascular channels can be detected using HR-pQCT, however due to the small size and heterogeneous presentation of these vascular channels, the detected number was limited and it appeared that the current definition was insufficient.

\section{Contributions of this thesis to society}

The findings in this thesis are of significant importance and of additional value for current understanding and further research in RA. If vascular channels play a role in the development of large cortical interruptions, the ability of HR-pQCT imaging to identify vascular channels in finger joints enables, in part, the detection and monitoring of these vascular channels. When large cortical interruptions indeed develop at the 'entry points' through vascular channels, HR-pQCT imaging will enable to show and follow these bone changes over time. This monitoring may be of additional value, also after treatment initiation in clinical studies in order to assess the effect of treatment on this process. Due to the high sensitivity of HR-pQCT, it is expected that fewer participants are needed in clinical trials with HR-pQCT compared to trials using conventional radiography, to observe an effect of for example drug treatment.

Some of the results in this thesis were obtained after collaboration with a team from Aarhus University, Aarhus, Denmark. Initially we met during international meetings organized by the SPECTRA (Study GrouP for XTrEme CT in RA) collaboration. Collaborations allow broader studies into for example, the mechanisms involved in pathological bone erosion development by combining different fields of expertise such as histology, cell research and HR-pQCT imaging. Therefore the value of the continuation of our collaboration shall be stressed out during annual meetings of SPECTRA. Furthermore, SPECTRA is a Special Interest Group (SIG) at the Outcome Measures in Rheumatology (OMERACT) initiative. This initiative aims to identify and improve relevant health outcome domains. An instrument such as HR-pQCT needs to meet the requirements of the OMERACT filters defined by "truth", "discrimination" and "feasibility". The members of the SPECTRA collaboration strive to implement the OMERACT approach in their studies. As such, the results of this thesis shall be presented during future OMERACT meetings.

\section{Future perspectives}

Currently, HR-pQCT has only been used in research settings. Further validation is needed to study the possibility of use in clinical settings. However, at the moment we also see opportunities to use HR-pQCT images for patient educational purposes. Research showed that three-dimensional reconstruction of joint prototypes helped patients to better understand the impact of a disease like RA on their bone ${ }^{8}$. Treatment adherence after showing the impact of the disease on bone could possibly be improved. To test this, an intervention study using three-dimensional prototypes of joints affected by RA, but also by other rheumatic disease such as psoriatic arthritis and gout, 
could be initiated. When newly diagnosed patients attend the outpatient clinic, research nurses can use the prototypes to explain what happens to the bone of the joints affected by the disease over time. In addition, three-dimensional reconstructions of some of their own joints can be made, which also makes HR-pQCT imaging more comprehensive to patients.

The results described in this thesis are based on cross-sectional data, but a follow-up was planned as part of these studies. Therefore, the longitudinal data that will become available may further contribute to knowledge about the importance of vascular channels and the development and progression of cortical interruptions in RA.

The HR-pQCT device used in this thesis is the first generation of HR-pQCT scanners. With the recent introduction of the second generation HR-pQCT scanner, the higher isotropic voxel size of this new scanner $(61 \mu \mathrm{m}$ versus $82 \mu \mathrm{m})$ can for example provide more details on the role of vascular channels in development of large cortical interruptions. Furthermore, the information on the role of vascular channels can be translated to other types of bone disease research, for example in psoriatic arthritis or osteoarthritis.

Last, in this thesis we showed that visual scoring of HR-pQCT images was time consuming, but that the automated algorithm showed promising results to sensitively and objectively detect cortical interruptions. Future research should focus on further validation of this automated algorithm.

This thesis provides a solid basis/foundation with interesting outcomes of HR-pQCT imaging of finger joints in healthy subjects and patients with RA, which can further be explored in future research.

\section{Current publicity}

Results in this thesis have been submitted and published in scientific journals. As part of the SPECTRA collaboration we published a supplement issue in the Journal of Rheumatology on HR-pQCT imaging. Results have also been presented at national and international conferences. Oral presentations have been given at the national conference of the Dutch Rheumatology association (Nederlandse Vereniging voor Reumatologie, 2014, 2015 and 2016). Posters have been presented at the European League Against Rheumatism (EULAR 2014, 2015 and 2016) and American College of Rheumatology (ACR 2014 and 2016). 


\section{References}

1 Paleolog, E. M. The vasculature in rheumatoid arthritis: cause or consequence? Int J Exp Patho/ 90, 249261 (2009).

2 Gravallese, E. M. \& Walsh, N. C. Rheumatoid arthritis: Repair of erosion in RA-shifting the balance to formation. Nat Rev Rheumatol 7, 626-628 (2011).

3 Lillegraven, S., van der Heijde, D., Uhlig, T., Kvien, T. K. \& Haavardsholm, E. A. What is the clinical relevance of erosions and joint space narrowing in RA? Nat Rev Rheumato/ 8, 117-120 (2012).

4 Ostergaard, M., Pedersen, S. J. \& Dohn, U. M. Imaging in rheumatoid arthritis-status and recent advances for magnetic resonance imaging, ultrasonography, computed tomography and conventional radiography. Best Pract Res Clin Rheumatol 22, 1019-1044 (2008).

5 Stach, C. M. et al. Periarticular bone structure in rheumatoid arthritis patients and healthy individuals assessed by high-resolution computed tomography. Arthritis Rheum 62, 330-339 (2010). Barnabe, C. et al. Definition for Rheumatoid Arthritis Erosions Imaged with High Resolution Peripheral Quantitative Computed Tomography and Interreader Reliability for Detection and Measurement. J Rheumatol 43, 1935-1940 (2016). Schett, G., Saag, K. G. \& Bijlsma, J. W. From bone biology to clinical outcome: state of the art and future perspectives. Ann Rheum Dis 69, 1415-1419 (2010). Kleyer, A. et al. Development of three-dimensional prints of arthritic joints for supporting patients' awareness to structural damage. Arthritis Res Ther 19, 34 (2017). 


\section{Nederlandse samenvatting}

Ongeveer $1 \%$ van de wereldbevolking wordt getroffen door reumatoïde artritis (RA), een chronische ziekte die gekenmerkt wordt door gewrichtsontstekingen'. De gewrichtsontstekingen leiden tot schade aan het bot van onder andere de vingergewrichten, en dit gebeurt soms al in een vroeg stadium van $\mathrm{RA}^{1,2}$. Deze schade in het bot, in de vorm van zogenaamde corticale interrupties (onderbreking van de buitenste rand van het bot) met verlies van onderliggende botstructuur, zijn kenmerkend voor RA. Op termijn kan deze schade zelfs leiden tot deformatie en verminderde functie van de vingergewrichten. Beeldvorming speelt daarom voor de diagnostiek en opvolging van RA een belangriike rol. Conventionele radiografie van de handen is binnen de klinische gezondheidszorg de gouden standaard ${ }^{3.7}$. Het maken van een röntgenfoto is gemakkelijk en snel. Echter, deze methode is niet heel gevoelig voor de detectie van schade aan het bot rondom de hand- en voetgewrichten, op de röntgenfoto erosie genoemd. De detectie van schade op een röntgenfoto wordt ook bemoeilijkt doordat de toename van schade aan het bot een langzaam proces is. Daarnaast neemt het scoren van röntgenfoto's veel tijd in beslag en kan het scoren gehinderd worden door overprojectie van de gewrichten,8-10. Bewezen gevoeligere beeldvormingstechnieken om vroegtijdig schade aan het bot op te sporen zijn computertomografie $(C T)$, echografie en magnetische resonantie imaging (MRI) ${ }^{1-14}$. In tegenstelling tot de röntgenfoto is het met echografie en MRI mogelijk de weke delen in beeld te brengen en ontsteking van het gewricht op te sporen 13,15-17. Met MRI kan ook beenmergoedeem (BME) zichtbaar gemaakt worden, dat wordt gezien als een belangrijke voorspeller van schade op de röntgenfoto ${ }^{15,18,19}$. Toch heeft het gebruik van enkel echografie en MRI ook nadelen. Bij echografie is de kundigheid van de operator belangrijk en is er een beperkte mogelijkheid om beeldopnamen dieper in het bot te maken. Voor MRI geldt dat opnamen lang duren, het gepaard gaat met hoge kosten in gebruik en in de praktijk vaak minder (snel) beschikbaar is.

Hoge resolutie perifere kwantitatieve CT (HR-pQCT) is een nieuwe techniek die gedetailleerde beelden van zowel corticaal bot (buitenste rand van het bot) als trabeculair bot (binnenste netwerk van botbalkjes) mogelijk maakt met een lage stralingsdosis (3 microSievert). Oorspronkelijk is HR-pQCT ontwikkeld om bot microarchitectuur in metabole botziekten gedetailleerd te bestuderen en om botsterkte te berekenen ${ }^{20-22}$. Recent is HR-pQCT ook toegepast voor onderzoek van bot rondom handgewrichten bii patiënten met RA. Naast het bestuderen van afwijkingen van het bot en het meten van corticale interrupties, is er ook onderzoek gedaan naar specifieke gebieden rondom de gewrichtsspleet, die gevoelig zijn voor ontwikkeling van corticale interrupties ${ }^{23-27}$. Eerdere studies legden de nadruk op grote interrupties van de cortex $(>1.9 \mathrm{~mm})$, soms met verlies van onderliggende trabeculaire structuur ${ }^{24}$. Onderzoek heeft aangetoond dat HR-pQCT ook sensitief is voor de detectie van kleine corticale interrupties, zonder dat sprake is van verlies van trabeculaire structuur $(<0.5 \mathrm{~mm})$ en dat deze kleine corticale interrupties ook gezien worden in de gezonde populatie ${ }^{24,25}$. 
Gesuggereerd is dat deze kleine corticale interrupties fysiologische vasculaire kanalen kunnen zijn'28. Volgens de "Study grouP for xtrEme Computed Tomography in Rheumatoid Arthritis" (SPECTRA) wordt een vasculair kanaal op HR-pQCT beelden gekenmerkt door een corticale interruptie met een parallelle structuur op twee opeenvolgende HR-pQCT beelden in twee vlakken (" $\left.2 \times 22^{\prime \prime}\right)^{29}$. Uit een recente studie (exvivo) met gebruik van barium als contrastmiddel bleek dat de voorgestelde definitie voor een vasculair kanaal met parallelle structuur correct was $s^{30}$. Echter, studies die gebruik maken van andere gouden standaarden zoals microCT $(\mu \mathrm{CT})$ of histologie, waren nog niet gedocumenteerd.

Het 'Outcomes Measures in Rheumatology' (OMERACT) initiatief is een onafhankelijk initiatief van professionals uit de gezondheidszorg die onderzoek doen naar vitkomstmaten binnen reumatologisch onderzoek. Volgens de OMERACT criteria moet een beeldvormingstechniek zoals HR-pQCT voldoen aan de eisen omschreven door de termen "waarheid", "discriminatie" en "bruikbaarheid"31,32. "Waarheid" wordt getoetst op basis van antwoorden op vragen als "Is de meting waardevol, meet het wat er bedoeld wordt?" en "Is het resultaat onbevooroordeeld en relevant?"31. "Discriminatie" wordt getoetst op basis van antwoorden op vragen als "Kan de meting een onderscheid maken tussen situaties"31. "Bruikbaarheid" wordt getoetst door antwoorden op vragen als "Kan de meting gemakkelijk toegepast worden, lettend op tijd, geld en interpreteerbaarheid?"31. Het gebruik van HR-pQCT beeldvorming van vingergewrichten in patiënten met RA is daarom in dit proefschrift getoetst aan de hand van deze criteria.

Voor de onderzoeken beschreven in dit proefschrift hebben we gebruik gemaakt van data vit een cohort bestaande vit 41 patiënten met RA en 38 gezonde proefpersonen, het zogenaamde MOSA-Hand cohort, gerekruteerd in het Maastricht Universitair Medisch Centrum. Tevens hebben we gebruikt gemaakt van vingers van overleden personen, beschikbaar gesteld voor de wetenschap (ex-vivo vingers).

\section{Samenvatting}

De onderzoeken zoals beschreven in dit proefschrift richten zich op de waarneming van corticale interrupties in het bot rondom de vingergewrichten van patiënten met RA en gezonde proefpersonen met behulp van HR-pQCT beelden. De waarheid en discriminatie van het visueel detecteren van corticale interrupties op HR-pQCT beelden met $\mu \mathrm{CT}$ als gouden standaard is onderzocht. Daarnaast hebben we in ex-vivo vingers gedetailleerd onderzoek verricht naar corticale interrupties, die op basis van HR-pQCT omschreven konden worden als een vasculair kanaal met als gouden standaard de histologische preparaten van deze ex-vivo vingers. Vervolgens werden in diezelfde studie karakteristieken van de op histologie aangeduide vasculaire kanalen geëvalueerd op enkel en meerdere opeenvolgende (multiplanair) HR-pQCT beelden. Aanvullend werd een visuele scoringsmethode voor het waarnemen van corticale 
interrupties op HR-pQCT ontwikkeld en getest op waarheid en bruikbaarheid. We gebruikten deze visuele methode om beelden van het MOSA-Hand cohort te evalueren, en bestudeerden mogeliike associaties tussen structurele schade op MRI en radiografie, kenmerken van inflammatie op MRI (zoals BME en synovitis), andere risico factoren (zoals antistoffen tegen gecitrullineerde eiwitten (ACPA) en/of reumafactor (RF), hand dominantie en ziekteduur) op de aanwezigheid en aantal corticale interrupties op HRpQCT. Tot slot werd een automatische scoringsmethode ontwikkeld en getest op waarheid en bruikbaarheid.

In hoofdstuk 2 werd een overzicht gepresenteerd van de beeldvorming met HR-pQCT in vingergewrichten van patiënten met RA en gezonde proefpersonen met corresponderende beelden op MRI en röntgenfoto's. Een selectie van beelden van vingergewrichten uit het MOSA-Hand cohort is hiervoor gebruikt. Er werd een heterogeen spectrum van corticale interrupties op HR-pQCT beelden in metacarpofalangeale (MCP) gewrichten van zowel patiënten met RA als ook gezonde proefpersonen gezien. Corticale interrupties met en zonder verlies van trabeculaire structuur, waarvan gesuggereerd wordt door SPECTRA dat ze ofwel fysiologische vasculaire kanalen zijn of pathologische erosies, werden gevonden in zowel de top van de metacarpaal als de basis van de falanx. Corticale interrupties werden gevonden in alle kwadranten, dat wil zeggen, de radiale, ulnaire, palmaire en dorsale kwadranten, en varieerden in grootte. Dit overzicht bevestigt de resultaten uit eerder onderzoek waarin werd beschreven dat de aanwezigheid en grootte van schade aan het bot op basis van de HR-pQCT beelden, groter en complexer is, dan gezien kan worden op röntgenfoto's 23,24,33-35. De klinische betekenis van de corticale interrupties gezien bij patiënten met RA en gezonde proefpersonen bleef onbekend.

Aan het begin van dit proefschrift was het onduidelijk in welke mate HR-pQCT betrouwbaar is als het gaat om weergave van kleine corticale interrupties en de interpretatie ervan. In hoofdstuk 3 werden daarom aspecten van betrouwbaarheid en validiteit van het gebruik van HR-pQCT in de visuele detectie van corticale interrupties in MCP en proximale interfalangeale (PIP) gewrichten onderzocht met $\mu \mathrm{CT}$ als gouden standaard. Tien ex-vivo vingers werden onderzocht met HR-pQCT en $\mu C T$ met resoluties van 82 en 18 micron. In totaal 19 gewrichten werden onafhankelijk van elkaar door twee beoordelaars beoordeeld op de aanwezigheid van corticale interrupties. De betrouwbaarheid tussen twee lezingen door één beoordelaar (intra) en de betrouwbaarheid tussen twee beoordelaars (inter), werden geëvalueerd voor de aanwezigheid van corticale interrupties door middel van Cohen's kappa $(\kappa)$ en intraclass correlatie coëfficiënt (ICC). Sensitiviteit, specificiteit, positieve en negatieve voorspellende waarden (PVW en NVW) van HR-pQCT in de detectie van corticale interrupties werden berekend. Het totaal aantal corticale interrupties gevonden in de 19 gewrichten bedroeg 129 (HR-pQCT) en $149(\mu \mathrm{CT})$. De betrouwbaarheid tussen beoordelaars voor de aanwezigheid van corticale interrupties en voor het totale aantal 
corticale interrupties voor zowel HR-pQCT als $\mu C T$ berekend op kwadrant niveau was "matig" tot "redelijk" (bereik 0.52 - 0.61). HR-pQCT bleek sensitief te zijn (81.6\%) om corticale interrupties te detecteren met $\mu \mathrm{CT}$ als gouden standaard, echter was de specificiteit laag (64.0\%). De PVW en NVW waren 81.6\% en 64.0\%. Daarom dienden de gedetecteerde corticale interrupties verder onderzocht te worden, het liefst door middel van histologisch onderzoek. Ook achtten we een betrouwbaardere definitie- en classificatiesysteem nodig om corticale interrupties te analyseren door middel van HR-pQCT.

Ons onderzoek werd daarom voortgezet door middel van een histologische studie om de achtergrond van corticale interrupties te bestuderen. In hoofdstuk 4 vergeleken we corticale interrupties op HR-pQCT beelden, die volgens de SPECTRA definitie geclassificeerd konden worden als vasculair kanaal, met beeldkarakteristieken op histologische preparaten. Hierna werden karakteristieken van op histologie gedefinieerde vasculaire kanalen geëvalueerd op enkel en meerdere, opeenvolgende gematchte HR-pQCT beelden. Vier voorgeselecteerde regio's vit drie MCP gewrichten van ex-vivo vingers werden eerst histologisch onderzocht. Het bleek dat van de zeven vasculaire kanalen op HR-pQCT volgens de SPECTRA definitie, maar één een echt histologisch bewezen vasculair kanaal was. Verder werden 52 (kleine) vasculaire kanalen gezien op histologie welke een heterogeen patroon lieten zien op corresponderende HR-pQCT beelden. Op corresponderende enkelvoudige doorsnedes van HR-pQCT beelden waren 25 van de 52 vasculaire kanalen volledige corticale interrupties die de cortex recht of schuin binnen drongen. Veertien van de 52 vasculaire kanalen hadden geen volledige interruptie van de cortex, maar presenteerde zich als een vitholling, sommige eenvoudig, andere complex van vorm. Op corresponderende multiplanaire HR-pQCT beelden waren 11 vasculaire kanalen te zien in minstens $2 \times 2$ opeenvolgende HR-pQCT beelden, en 36 vasculaire kanalen in minder dan 2x2 opeenvolgende beelden. Niet alle histologisch gedetecteerde vasculaire kanalen konden worden gezien op enkel- of multiplanaire HR-pQCT beelden (respectievelijk 13 en 5 vasculaire kanalen). De diameter van de vasculaire kanalen varieerde van 0.049 tot $0.790 \mathrm{~mm}$, waar 6 van de 52 vasculaire kanalen kleiner waren dan de resolutie van HR-pQCT $(82 \mu \mathrm{m})$. Uit ons onderzoek blijkt dat de heterogene presentatie van histologisch geïdentificeerde vasculaire kanalen, de kleine diameter van een aantal van deze vasculaire kanalen en de resolutie van HR-pQCT de detectie van vasculaire kanalen op HR-pQCT beelden limiteert. Verder bleek de voorgestelde SPECTRA definitie niet voldoende om vasculaire kanalen op HR-pQCT beelden adequaat te detecteren.

Kleine corticale interrupties waren tot op heden nog niet meegenomen in de voorgestelde scoringsmethoden voor HR-pQCT beelden. Aangezien we in hoofdstuk 3 aantoonden dat HR-pQCT sensitief is om ook kleine corticale interrupties weer te geven, ontwikkelden en valideerden we in hoofdstuk 5 een visuele scoring methode voor het detecteren van corticale interrupties in vingergewrichten op HR-pQCT beelden. Uit het 
MOSA-hand cohort werden 30 proefpersonen 110 gezonde proefpersonen en 20 patiënten met RA) geselecteerd, gebaseerd op radiologische schade volgens de Sharp van der Heijde radiografische score. De dataset bestond uit in totaal 98 gewrichten: 20 gewrichten van gezonde proefpersonen en 78 van patiënten met RA. Het voorstel voor deze visuele scoringsmethode hebben we gebaseerd op eerder verschenen publicaties over gradaties in bewegingsartefacten ${ }^{36}$, het scoren van gewrichten ${ }^{24}$, discussies met experts, consensus bijeenkomsten en meerdere scoringsoefeningen. Het team van experts bestond uit reumatologen, radiologen en ingenieurs die gespecialiseerd zijn in beeldanalyse. De visuele scoringsmethode omvatte ook de kleine corticale interrupties, die te zien zijn op één vlak en daarbii in twee vlakken $(" 1 \times 2$ "), met of zonder achterliggende trabeculaire verstoring. Betrouwbaarheid en bruikbaarheid van het visuele scoringsalgoritme werden onderzocht. In totaal werden 252 corticale interrupties gezien (diameter variërend van $0.09 \mathrm{~mm}$ tot $7.40 \mathrm{~mm}$ ) in zowel gezonde proefpersonen ( $n=38)$ als patiënten met RA $(n=214)$. Echter, in patiënten met RA werd in $21 \%$ van de corticale interrupties trabeculaire verstoring gezien. Bij gezonden werden er geen corticale interrupties met trabeculaire verstoring gezien. In vergelijking met gezonde proefpersonen hadden patiënten met RA significant meer corticale interrupties in zowel het ulnaire als in het radiale kwadrant (beide kwadranten $p<0.05)$. In gezonde proefpersonen en patiënten met RA werden ook frequent corticale interrupties gezien in het palmaire kwadrant. De intra-beoordelaar betrouwbaarheid voor de aanwezigheid ( $\mathrm{k}$ ) en totaal aantal (ICC) corticale interrupties was "redelijk" tot "bijna perfect" op gewrichtsniveau (bereik 0.52 - 0.88) en "voldoende tot goed" op kwadrantniveau (bereik 0.63 - 0.69). De inter-beoordelaar betrouwbaarheid voor de aanwezigheid en totaal aantal corticale interrupties was "matig" tot "redelijk" op gewrichtsniveau (bereik $0.37-0.48$ ) en "redelijk" per kwadrant (bereik $0.53-0.56$ ). Voor het uitvoeren van de visuele scoringsmethode waren gemiddeld 9.2 (SD 4.9) minuten nodig. Concluderend, de visuele scoringsmethode staat toe ook kleine corticale interrupties in vingergewrichten op HR-pQCT beelden mee te nemen. Ondanks dat de vitvoering van de methode nog tijdrovend is, lijkt de methode veelbelovend voor het gebruik in klinische studies.

In hoofdstuk 6 pasten we de visuele scoring methode toe op de HR-pQCT beelden van het MOSA-Hand cohort. Mogelijke associaties tussen structurele schade op MRI en radiografie, kenmerken van inflammatie op MRI (zoals BME en synovitis), andere risicofactoren (ACPA en/of RF, handdominantie en ziekteduur) met de aanwezigheid en aantal corticale interrupties op HR-pQCT werden bestudeerd. In totaal werden de vingergewrichten van 38 gezonde proefpersonen en 39 patiënten met RA onderzocht door middel van radiografie, MRI en HR-pQCT. Radiografische schade werd beoordeeld middels de Sharp/ van der Heijde methode. MRI beelden werden beoordeeld op aanwezigheid van corticale interrupties, BME en synovitis. HR-pQCT beelden werden beoordeeld op de aanwezigheid van corticale interrupties. Een significante associatie werd gezien tussen structurele schade op MRI alsook radiografie 
met de aanwezigheid van corticale interrupties op HR-pQCT (respectievelijk odds ratio (OR) 12.4, 95\% betrouwbaarheidsinterval (BI) 7.5-21.4, $\mathrm{p}<0.01$ en OR 4.8, 95\% BI 1.9-11.7, $p<0.011$. De aanwezigheid van BME of synovitis op MRI was geassocieerd met meer corticale interrupties op HR-pQCT (Beta 0.47, 95\% BI 0.4-0.6, p<0.01 en Beta 1.9, 95\% BI 0.6-3.1, p<0.01). Bij patiënten met RA waren in deze studie ACPA en/of RF seropositiviteit, handdominantie en ziekteduur niet significant geassocieerd met meer corticale interrupties op HR-pQCT.

In hoofdstuk 7 werd een volledig automatisch algoritme om corticale interrupties op HR -pQCT te detecteren ontwikkeld en op betrouwbaarheid getest. Ook het effect van manuele correctie van de automatisch gegenereerde contouren (semiautomatisch) werd getest. HR-pQCT beelden van $10 \mathrm{MCP}$ gewrichten (zeven uit patiënten met RA en drie vit gezonde proefpersonen) werden geselecteerd vit het MOSA-hand cohort. Het totaal aantal corticale interrupties en het oppervlakte van de corticale interrupties per gewricht werden geëvalueerd. Er werd geen significant verschil in het totaal aantal corticale interrupties gevonden tussen het volledige en semiautomatische algoritme gevonden (mediaan 15 versus mediaan 14, $\mathrm{p}=0.37$ ). Bij vergelijking van de resultaten van het volledig automatische en semiautomatisch algoritme, werd een bijna perfecte betrouwbaarheid gezien (ICC 0.95 - 0.97). Het volledig geautomatiseerde algoritme is daarom een veelbelovende methode om corticale interrupties op HR-pQCT beelden van vingergewrichten te detecteren. De handmatige correctie tijdens de semiautomatische methode had weinig meerwaarde. Echter, wordt deze vooralsnog aanbevolen om de kwaliteit van de resultaten te waarborgen. Verdere validatie van de volledig automatische methode in klinische studies is nodig om de potentiele waarde van de methode te onderzoeken, mede omdat de huidige studie cross-sectionele data gebruikte. De mogelijkheid om met het algoritme progressie van schade aan het bot gedurende een bepaalde tijdperiode te meten moet nader worden onderzocht.

In hoofdstuk 8 werden de belangrijkste bevindingen uit dit proefschrift samengevat en bediscussieerd. Er werd teruggekomen op de door OMERACT vastgestelde termen "waarheid", "discriminatie" en "bruikbaarheid".

Bij "waarheid" bleek dat HR-pQCT sensitief is in de detectie van corticale interrupties. Ook bleek dat HR-pQCT in staat is om een heterogeen patroon van histologisch gedetecteerde vasculaire kanalen weer te geven. De voorgestelde definitie van SPECTRA bleek echter tekort te schieten. Het formuleren van een nieuwe definitie voor vasculaire kanalen op HR-pQCT zal een uitdaging zijn, gezien de kleine diameter van vele vasculaire kanalen en de resolutie van HR-pQCT die de detectie van alle vasculaire kanalen limiteert. "Waarheid" werd verder onderzocht in hoofdstuk 6 waar met behulp van de visuele scoringsmethode significant meer corticale interrupties gezien werden in patiënten met RA dan in gezonden en een significante relatie gezien werd tussen schade op MRI en handenfoto met de aanwezigheid van corticale interrupties op HRpQCT. We maakten in hoofdstuk 6 echter geen onderscheid tussen de corticale 
interrupties, dat wil zeggen mogelijke fysiologische vasculaire kanalen of pathologische erosies. Toekomstig onderzoek, het liefst met behulp van histologie, kan de verdere achtergrond van corticale interrupties bestuderen.

Bij "discriminatie" werd gekeken naar de betrouwbaarheid van beoordelingen. In hoofdstuk 3 en 5 werden intra- en inter-beoordelaar betrouwbaarheid geëvalueerd voor de detectie van corticale interrupties op HR-pQCT in vergelijking met $\mu \mathrm{CT}$ (hoofdstuk 3) en voor de visuele scoringsmethode (hoofdstuk 5). De intra-beoordelaar betrouwbaarheid bleek "redelijk" tot "voldoende tot goed" voor de aanwezigheid van een corticale interruptie en "voldoende tot goed" tot "bijna perfect" voor het totaal aantal corticale interrupties. De inter-beoordelaar betroumbaarheid voor beide studies was "matig" tot "redelijk" voor de aanwezigheid van een corticale interruptie en "redelijk" voor het totaal aantal corticale interrupties. Visuele beoordeling van beelden blijft moeilijk, zelfs door ervaren beoordelaars ${ }^{37}$. Deze limitatie moet in overweging genomen worden wanneer een scoringsmethode ontwikkeld wordt, of onze visuele scoringsmethode wordt gebruikt. In beide studies uit hoofdstuk 3 en 5 werden de grote verschillen tussen beoordelaars geëvalueerd. Ten eerste bleek dat bij kleinere corticale interrupties minder overeenstemming was. Ten tweede maakten de lage mineralisatie en/of de dunne cortex het soms lastig de corticale interruptie te onderscheiden van de achtergrond ruis. Ten derde bleek dat hoewel grotere interrupties meer overeenstemming hadden, in sommige gevallen meerdere kleine interrupties werden geteld binnen één grote interruptie. De betrouwbaarheid zou verbeterd kunnen worden door enkel grote interrupties te tellen, maar de kracht van HR-pQCT is juist om ook kleinere corticale interrupties weer te geven. $\mathrm{Er}$ is dus een betrouwbaardere methode dan een visuele scoringsmethode nodig om corticale interrupties waar te nemen. In hoofdstuk 7 bleek een automatisch algoritme veelbelovend met bijna perfecte betrouwbaarheid. Het automatische algoritme detecteerde kleinere corticale interrupties dan gezien werden met eerdere visuele algoritmes ${ }^{38,39}$. Deze resultaten maken het algoritme interessant om mogelijk in klinische studies te gebruiken, maar de mogelijkheid van het algoritme om de progressie van schade over de tijd te meten, moet nog onderzocht worden.

Bii "bruikbaarheid" werd gekeken naar de vitvoerbaarheid van de visuele scoring methode op HR-pQCT beelden. Met een gemiddelde tijd van 9.2 minuten (minimaal 1 tot maximaal 38 minuten) is de visuele methode niet uitvoerbaar in de klinische setting. De voorgestelde automatische methode was veelbelovend, en kan in enkele minuten worden vitgevoerd.

De resultaten beschreven in dit proefschrift laten zien dat HR-pQCT betrouwbaar en sensitief is voor het detecteren van corticale interrupties in bot rondom vingergewrichten. In de toekomst kan HR-pQCT beeldvorming van de vingers wellicht gebruikt worden in klinische trials, om de effecten van therapieën te monitoren en evalueren. Ook kan het interessant zijn HR-pQCT te gaan gebruiken om afwijkingen of veranderingen van het bot in de vingers te meten bij andere reumatische aandoeningen 
zoals jicht en artrose. Dit proefschrift biedt een solide basis voor toekomstig onderzoek met HR-pQCT, waar de focus zal liggen op de klinische relevantie van corticale interrupties door middel van longitudinaal onderzoek, en de optimalisatie van (semi) automatische algoritmen om corticale interrupties te detecteren. 


\section{Referenties}

1 Paleolog, E. M. The vasculature in rheumatoid arthritis: cause or consequence? Int J Exp Pathol 90, 249 261 (2009).

2 van der Heijde, D. M. Joint erosions and patients with early rheumatoid arthritis. Br J Rheumatol 34 Suppl 2, 74-78 (1995).

Boini, S. \& Guillemin, F. Radiographic scoring methods as outcome measures in rheumatoid arthritis: properties and advantages. Ann Rheum Dis 60, 817-827 (2001).

Lillegraven, S., van der Heijde, D., Uhlig, T., Kvien, T. K. \& Haavardsholm, E. A. What is the clinical relevance of erosions and joint space narrowing in RA? Nat Rev Rheumato/ 8, 1 17-120 (2012).

Ravindran, V. \& Rachapalli, S. An overview of commonly used radiographic scoring methods in rheumatoid arthritis clinical trials. Clin Rheumato/ 30, 1-6 (2011).

Sommer, O. J. et al. Rheumatoid arthritis: a practical guide to state-of-the-art imaging, image interpretation, and clinical implications. Radiographics 25, $381-398$ (2005).

van der Heijde, D., Dankert, T., Nieman, F., Rau, R. \& Boers, M. Reliability and sensitivity to change of a simplification of the Sharp/van der Heijde radiological assessment in rheumatoid arthritis. Rheumatology (Oxford) 38, 941-947 (1999).

Mottonen, T. T. Prediction of erosiveness and rate of development of new erosions in early rheumatoid arthritis. Ann Rheum Dis 47, 648-653 (1988).

Nissila, M. et al. Prognosis of inflammatory joint diseases. A three-year follow-up study. Scand J Rheumatol 12, 33-38 (1983).

van der Heijde, D. M. et al. Biannual radiographic assessments of hands and feet in a three-year prospective followup of patients with early rheumatoid arthritis. Arthritis Rheum 35, 26-34 (1992).

11 Ostergaard, M., Pedersen, S. J. \& Dohn, U. M. Imaging in rheumatoid arthritis-status and recent advances for magnetic resonance imaging, ultrasonography, computed tomography and conventional radiography. Best Pract Res Clin Rheumatol 22, 1019-1044 (2008).

( radiography for the detection of bone erosions in rheumatoid arthritis patients: a systematic review and meta-analysis. Rheumatology (Oxford) 50, $1137-1147$ (2011). erosions? A comparison with computed tomography in rheumatoid arthritis metacarpophalangeal joints. Arthritis Res Ther 8, R 110 (2006).

Perry, D. et al. Detection of erosions in the rheumatoid hand; a comparative study of multidetector computerized tomography versus magnetic resonance scanning. J Rheumato/ 32, 256-267 (2005). Conaghan, P. G. et al. Elucidation of the relationship between synovitis and bone damage: a randomized magnetic resonance imaging study of individual joints in patients with early rheumatoid arthritis. Arthritis Rheum 48, 64-71 (2003).

Ostergaard, M. et al. Magnetic resonance imaging-determined synovial membrane and joint effusion volumes in rheumatoid arthritis and osteoarthritis: comparison with the macroscopic and microscopic appearance of the synovium. Arthritis Rheum 40, 1856-1867 (1997).

stendorf, B et al Magnetic resonance imaging and miniarthroscopy of metacarpophalangeal joints: sensitive detection of morphologic changes in rheumatoid arthritis. Arthritis Rheum 44, 2492-2502 (2001). McGonagle, D. et al. The relationship between synovitis and bone changes in early untreated rheumatoid arthritis: a controlled magnetic resonance imaging study. Arthritis Rheum 42, 1706-1711 (1999). Krabben, A. et al. MRI-detected subclinical joint inflammation is associated with radiographic progression. Ann Rheum Dis 73, 2034-2037 (2014). Boutroy, S., Bouxsein, M. L., Munoz, F. \& Delmas, P. D. In vivo assessment of trabecular bone microarchitecture by high-resolution peripheral quantitative computed tomography. J Clin Endocrinol Metab 90, 6508-6515 (2005).

Laib, A., Hauselmann, H. J. \& Ruegsegger, P. In vivo high resolution 3D-QCT of the human forearm. Technol Health Care 6, 329-337 (1998).

MacNeil, J. A. \& Boyd, S. K. Accuracy of high-resolution peripheral quantitative computed tomography for measurement of bone quality. Med Eng Phys 29, 1096-1105 (2007). Fouque-Aubert, A. et al. Assessment of hand bone loss in rheumatoid arthritis by high-resolution peripheral quantitative CT. Ann Rheum Dis 69, 1671-1676 (2010). Stach, C. M. et al. Periarticular bone structure in rheumatoid arthritis patients and healthy individuals assessed by high-resolution computed tomography. Arthritis Rheum 62, 330-339 (2010).

Srikhum, W. et al. Quantitative and semiquantitative bone erosion assessment on high-resolution peripheral quantitative computed tomography in rheumatoid arthritis. J Rheumato/ 40, 408-4 16 (2013). Barnabe, C. \& Feehan, L. High-resolution peripheral quantitative computed tomography imaging protocol for metacarpophalangeal joints in inflammatory arthritis: the SPECTRA collaboration. J Rheumatol 39, 1494-1495 (2012).

Topfer, D., Finzel, S., Museyko, O., Schett, G. \& Engelke, K. Segmentation and quantification of bone erosions in high-resolution peripheral quantitative computed tomography datasets of the metacarpophalangeal joints of patients with rheumatoid arthritis. Rheumatology (Oxford) 53, 65-71 (2014).

8 Finzel, S. et al. A detailed comparative study of high-resolution ultrasound and micro-computed 
tomography for detection of arthritic bone erosions. Arthritis Rheum 63, 1231-1236 (2011).

Barnabe, C. et al. Definition for Rheumatoid Arthritis Erosions Imaged with High Resolution Peripheral Quantitative Computed Tomography and Interreader Reliability for Detection and Measurement. J Rheumatol 43, 1935-1940 (2016).

Boers, M., Brooks, P., Strand, C. V. \& Tugwell, P. The OMERACT filter for Outcome Measures in Rheumatology. J Rheumato/ 25, 198-199 (1998).

Barnabe, C., Szabo, E., Martin, L., Boyd, S. K. \& Barr, S. G. Quantification of small joint space width, periarticular bone microstructure and erosions using high-resolution peripheral quantitative computed tomography in rheumatoid arthritis. Clin Exp Rheumatol 31, 243-250 (2013).

34 Hecht, C. et al. Additive effect of anti-citrullinated protein antibodies and rheumatoid factor on bone erosions in patients with RA. Ann Rheum Dis (2014).

35 Lee, C. H. et al. Correlation of structural abnormalities of the wrist and metacarpophalangeal joints evaluated by high-resolution peripheral quantitative computed tomography, 3 Tesla magnetic resonance imaging and conventional radiographs in rheumatoid arthritis. Int J Rheum Dis (2014).

36 Pialat, J. B., Burghardt, A. J., Sode, M., Link, T. M. \& Majumdar, S. Visual grading of motion induced image degradation in high resolution peripheral computed tomography: impact of image quality on measures of bone density and micro-architecture. Bone 50, $111-118$ (2012). Sharp, J. T. et al. Variability of precision in scoring radiographic abnormalities in rheumatoid arthritis by experienced readers. J Rheumato/ 31, 1062-1072 (2004).

38 Finzel, S. et al. Repair of bone erosions in rheumatoid arthritis treated with tumour necrosis factor inhibitors is based on bone apposition at the base of the erosion. Annals of the rheumatic diseases 70, 1587-1593 (2011).

Finzel, S. et al. Interleukin-6 receptor blockade induces limited repair of bone erosions in rheumatoid arthritis: a micro CT study. Annals of the rheumatic diseases 72, 396-400 (2013). 


\section{Dankwoord}

Het heeft iets langer dan vier jaar geduurd, maar de eindstreep is gehaald. Dank aan allen die bijgedragen hebben aan dit proefschrift. In het bijzonder dank aan de vrijwillige inzet van de proefpersonen, zonder hen geen data. Verder wil ik ook bij naam de volgende personen bedanken:

Mijn promotie team, Prof. dr. Geusens, Piet, Prof. dr. van den Bergh, Joop, Dr. van Tubergen, Astrid en Dr. ir. van Rietbergen, Bert.

Ik citeer eerst een zin uit het eerste email contact wat ik met Astrid over dit promotietraject had; "Het is een zeer vitdagend project, echt pionierswerk, want er zijn nog nauwelijks studies hiernaar gedaan (19-11-2012)". En dit was correct, uitdagend in diverse opzichten, soms terug bij af. We vonden een richting voor dit proefschrift en we brachten verschillende disciplines samen. Het resultaat ligt hier nu in de vorm van mijn proefschrift. Eigenlijk zijn we nog niet klaar en zoals beschreven in de discussie legt dit proefschrift een basis voor verder onderzoek. Bedankt voor het inzetten van jullie specifieke kennis en kunde.

Overige leden van de "handenwerkgroep", maandelijks kwamen we samen om de updates te delen en bespreken en soms ook om vitvoerig te discussiëren; Dr. Weijers, René en Dr. Loeffen, Daan, bedankt voor jullie inzet, commentaren en uiteraard voor het lezen van de MRI beelden. Dr. C. Arts, Chris, bedankt voor je input.

Other co-authors, thank you for the cooperation and valuable input.

Leden van de beoordelingscommissie:

Prof. dr. L. van Rhijn, Prof. dr. R. van der Hulst, Prof. dr. K. Ito, en Prof. dr. G. Kloppenburg, bedankt voor jullie moeite en inzet om mijn proefschrift te beoordelen. Prof. dr. Boonen, Annelies, als hoofd van de afdeling kende ik $u$ al; bedankt voor uw enthousiaste betrokkenheid bii de promovendi.

Bii de metingen van de proefpersonen kwam een hoop logistiek kijken. Het was altijd spannend de proefpersonen op tijd op de juiste plekken te krijgen. Laboranten MRI en röntgen: bedankt! Roland Kersemakers en Kim Feron, bedankt voor jullie inzet bij het verwerken van alle $\mathrm{MRI} /$ röntgen aanvragen, het aanpassen en schuiven met de planning. Ook bedankt Biobank Maastricht voor het verwerken van de monsters.

Het grootste deel van de tijd heb ik doorgebracht met mijn collega's op UNS40 en ja in de laatste 3 weken UNS50....

Antje, Honey Badger takes what it wants, avonturen in de Mulder Bergsport en vitstapjes naar de Bever, matchende pyjama's in Parijs, en wat hebben we een vermogen uitgegeven aan croissanties en koffie bij Ineke van de Douwe Egberts. In het vervolg moeten we een stukje rijden om te daten, maar het gaat je goed in Oostenrijk. 
Bart, vit het oog is niet altijd uit het hart, de route naar de goede koffie bewandelde je nog regelmatig.

Carmen, samen verkende we het datecircuit, of was Lexa toch een makelaar site?

Casper, ons fotomodel. Na 3 jaar attendeerde iij mij op het bestaan van een panoramische route in het ziekenhuis.

Ellis, met jou betekent leven in de brouwerii, altijd in voor eten, drinken, film of wat voor een vitstapje ook.

Frans, <5u22 in 2018? Gelukkig hebben we dat mooie filmpie van onze vakantie in Zweden.

Ivette, Hugo in de stad of Cheesecake Factory in Boston, een beetje zoals Ellis, iii bent altiid te porren voor iets.

Joost, ik leerde je kennen als held die het aandurfde om met 4 vrouwen op wintersport te gaan. Verder was je altijd in voor frietjes of sushi, al at ik net zo veel als iij.

José, wat was ik jaloers dat je naar Vancouver ging, maar daardoor kreeg je buikkastie wel een mooi nieuw thuis.

Lieke, wanneer je vanuit Zweden weer eens naar Maastricht kwam wist ik dat wordt uit eten! Gelukkig voor onze portemonnee bestaat Social Deal. Een postkantoor in Zweden bleek ook handig om mijn triatlonpakje naar Nederland te kriigen.

Lisanne, we deden nog zo ons best bij Frans in de keuken maar goed, dan maar ontspannen in Thermae 2000 met nacho's.

Mayke, kamergenoot na het vertrek van Simon en Antje, leuk dat je ook het fietsvirus te pakken kreeg.

Michiel, lekker op z'n Brabants met Hawaii broek in Lyon, zolang er pils is, is het goed.

Polina, jou doorzettingsvermogen om toch elke keer de trap naar de $5^{\mathrm{e}}$ te nemen daar kan ik een voorbeeld aannemen.

Yannick, ook al hoorde je meer bij farmacologie, vitjes met ons team waren leuker!

Cindy en Fiona, op de valreep werden we nog collega's, ik hoop dat jullie net zo'n leuke tijd zullen krijgen als ik hier beleefd heb.

Verdere collega's van de reumatologie:

Thea en Debby, bedankt voor jullie inzet om de handenfoto's te scoren. Marloes, Sandrine, Simone, Christel, en de rest van de staf die door de jaren heen aanwezig was, bedankt voor het helpen bij de inclusie van de patiënten en het verwerven van de nodige informatie. Het bleek dat ik met sommige van jullie nog meer gemeen had, zoals het kijken naar het geweldige programma "Ik vertrek"!

Janine (en Els), bedankt voor het bloedprikken bij de proefpersonen, en het helpen bij de voorbereiding van de audit. Yvonne, Peggy en in het begin ook nog Marian: duizendpoten op het secretariaat, bedankt!

Paranifmen Simon en Claudia, beter bekend als onderdeel van de Master Matties. Simon, op een dag belde je, er kwam een promotieplek op de afdeling en ze zochten 
nog iemand. Ik mocht zelfs bij jou en Antje op de kamer, een droomsamenstelling op kamer C5.53 la, en een tijd om nooit te vergeten.

Claudia, ook wij bleven elkaar onder werktijd zien, maar naast dit zakelijk contact waardeer ik onze vrije tijd met onder andere etentjes zeer. Niet alleen lekker kletsen of discussiëren maar ook kun je bij jou terecht voor raad en daad.

Fijn en bijzonder dat jullie tijdens mijn promotie mijn paranimfen willen zijn!

Ja en de overige Master Matties: Sanne, we zijn inmiddels een paar Rock Werchter edities verder. Naast het bezoeken van festivals en het maken van bijzondere reizen werk je ook nog hard, altijd met een lach, en jou positivisme werkt aanstekelijk. Rick, ik ben fan van de boerderijcamping, volgende keer organiseren we daar een spelletjes weekend. Gill, volgens mij lag de basis van deze Master Mattieformatie bij jou thuis, toen aan de Bergerstraat. Op naar een reünie.

Lieve vrienden en familie, niet iedereen is bekend met het fenomeen "promoveren". Hopelijk is het na het lezen van dit proefschrift lof ok, alleen de Nederlandse samenvatting misschien of alleen het dankwoord) een beetje helder. Fijn dat ik jullie om mij heen heb: gezelligheid, ontspanning, (sportieve/buitenland) tripjes, en een luisterend oor.

$\mathrm{Pa}$, ma en Bianca, voor jullie was het zeker niet erg dat er een slaapplek in Maastricht kwam. Frequente vitjes naar het Bourgondische andere zuiden van Nederland. Fijn dat jullie er zijn, voor support en ontspanning!

Michaël, bij sommigen bekend als de Bever of Bear Grylls, prima planning om mij te leren kennen in de eindfase van mijn proefschrift. Verbaasd over de verdwenen letters op mijn toetsenbord, maar komende tijd ben ik een stuk minder "cavia". 


\section{Curriculum Vitae}

Andrea Scharmga werd geboren op 8 december 1986 in Terneuzen. Na het behalen van haar VWO diploma aan het Zeldenrust-Steelantcollege in 2005 met het profiel Economie \& Maatschappii begon ze aan het University College Utrecht aan de bachelor Liberal Arts \& Sciences. Hier volgde ze voornamelijk "pre-medical" courses met een minor in psychologie. Haar master "Physical Activity \& Health" volgde ze vanaf 2008 aan Maastricht University. In het voorjaar van 2011 kreeg ze haar diploma voor de specialisatie "Biology of Human Performance \& Health" met extra vakken uit "Sports and Physical Activity Interventions" en "Cancer prevention". Haar studententijd werd ook nog opgevuld met een voltijds bestuursfunctie als voorzitter van Sportraad MUSST (2008-2009), en een parttime functie als commissaris extern bij de Maastrichtse studententennisvereniging Stennis (2009-2010). Na wat omzwervingen als onder andere skileraar in Oostenrijk, begon zij haar promotieonderzoek in 2013 op de afdeling reumatologie van het Maastricht Universitair Medisch Centrum, onder leiding van Prof. dr. P.P.M.M. Geusens, Prof. dr. J.P van den Bergh, Dr. A. van Tubergen en Dr. ir. B. van Rietbergen. De resultaten van haar onderzoek werden gepresenteerd op nationale en internationale congressen en resulteerden in dit proefschrift. Via het Clinical Trial Center Maastricht heeft ze tegenwoordig een nieuwe werkplek gevonden als onderzoeker bij de afdeling Plastische Chirurgie in samenwerking met Prof. dr. R. van der Hulst en Drs T. van Mulken. 
